\title{
Decomposição de Grupos de Dualidade de Poincaré, Obstruções sing e Invariantes Cohomológicos
}

Maria Paula dos Santos Cavalcanti

Dissertação de Mestrado

Pós-Graduação em Matemática 


\title{
Decomposição de Grupos de Dualidade de Poincaré, Obstruções sing e Invariantes Cohomológicos
}

\author{
Maria Paula dos Santos Cavalcanti
}

Dissertação apresentada ao Instituto de Biociências, Letras e Ciências Exatas da Universidade Estadual Paulista "Júlio de Mesquita Filho", Campus de São José do Rio Preto, São Paulo, para a obtenção do título de Mestre em Matemática.

Orientadora: Profa. Dra. Ermínia de Lourdes Campello Fanti 


\title{
Maria Paula dos Santos Cavalcanti
}

\author{
Decomposição de Grupos de Dualidade de Poincaré, Obstruções \\ sing e Invariantes Cohomológicos
}

Dissertação apresentada para obtenção do título de Mestre em Matemática do Instituto de Biociências, Letras e Ciências Exatas da Universidade Estadual Paulista "Júlio de Mesquita Filho", Campus de São José do Rio Preto.

COMISSÃO EXAMINADORA

TITULARES

Orientadora

Profa. Dra. Ermínia de Lourdes Campello Fanti

UNESP - São José do Rio Preto

Primeiro Examinador

Profa. Dra. Denise de Mattos

USP - São Carlos

Segundo Examinador

Profa. Dra. Maria Gorete Carreira Andrade

UNESP - São José do Rio Preto

\section{SUPLENTES}

Prof.Dr. Pedro Luiz Queiroz Pergher UFSCar - São Carlos

Profa.Dra. Luciana de Fátima Martins UNESP - São José do Rio Preto

São José do Rio Preto, 26 de Fevereiro de 2010. 
"Tudo que é reto mente.

Toda verdade é sinuosa.

O próprio tempo é um círculo."

Friedrich Nietzsche 
Aos meus pais,

José Naide e Eleonora, ofereço. 


\section{Agradecimentos}

Agradeço primeiramente à Deus por tudo que passei, pelo que aprendi ao longo desses anos, por ter me dado força quando pensei em desistir e por todas as pessoas especiais que colocou em minha vida, dentre elas em especial agradeço:

- À minha orientadora Profa. Ermínia, com muito carinho, pela dedicação, carinho e paciência com que me orientou desde a graduação, e sem a qual esse trabalho não teria se realizado.

- À meus amados pais, José Naide e Eleonora, meu querido irmão Genaildo e minhas queridas irmãs Edjane e Vivianne, pelo apoio, presença e alegria em minha vida.

- Aos professores do departamento pela formação.

- À todas as pessoas especiais que passaram pela minha minha ao longo desse período, às que passaram só de passagem e as muitas que ficaram.

- À CAPES pelo auxílio financeiro. 


\section{Resumo}

O objetivo principal deste trabalho é estudar as obstruções "sing "que desempenham papel importante nas demonstrações de certos resultados sobre decomposição de grupos que satisfazem certas hipóteses de dualidade apresentados em [16] e [17], em particular, sobre decomposição de um grupo $G$ adaptada a uma família $\mathcal{S}$ de subgrupos de $G$, com $(G, \mathcal{S})$ um par de dualidade de Poincaré. Alguns invariantes cohomológicos e certos resultados envolvendo tais invariantes, decomposição de grupos e/ou grupos e pares de dualidade são também apresentados.

Palavras-chave: Decomposição de grupos, cohomologia relativa de grupos, obstruções sing, grupos e pares de dualidade de Poincaré. 


\section{Abstract}

The main objective of this work is to study the obstructions "sing "which play an important role in demonstrating certain results on the splitting of groups that satisfy certain hypotheses of duality presented in [16] and [17], in particular, the decomposition of a group $G$ adapted to a family $\mathcal{S}$ of subgroups of $G$ with $(G, \mathcal{S})$ a Poincaré duality pair. Some cohomological invariants and certain results involving such invariants, a splitting of groups and/or groups and pairs of duality are also presented.

Keywords: Splitting of groups, relative cohomology of groups, obstructions sing, Poincaré duality groups and pairs. 


\section{SUMÁRIO}

Introdução $\quad$ x

1 Preliminares 1

1.1 Complexos de Cadeias e Cocadeias . . . . . . . . . . . . . . . . 1

1.2 Anel grupo e $\mathcal{R} G$-módulos . . . . . . . . . . . . . . . . . . . . . . . . . . . 10

1.3 Resoluções Livres e Projetivas . . . . . . . . . . . . . . . . . . . . . . . . . . . . . . . . . . . . . . . .

1.4 Módulo de Homomorfismos . . . . . . . . . . . . . . . . . . . . . . 21

1.5 Produto Tensorial . . . . . . . . . . . . . . . . . . 25

2 Grupos de (Co)homologia Absoluta e Relativa $\quad 27$

2.1 Grupos de (Co)homologia Absoluta . . . . . . . . . . . . . . 27

2.1.1 Restrição e (Co)extensão de Escalares . . . . . . . . . . . . . . . . . 31

2.1.2 Módulos Induzidos e Módulos Coinduzidos . . . . . . . . . . . . . . 33

$2.2 H^{*}$ como funtor de duas variáveis . . . . . . . . . . . . . . . . . 35

2.2.1 Aplicação Restrição . . . . . . . . . . . . . . . . . . . . . . 38

2.3 Grupos de (Co)homologia Relativa . . . . . . . . . . . . . . . . . 38

3 Dualidade de Poincaré $\quad 47$

3.1 Grupos e Pares de Dualidade . . . . . . . . . . . . . . . 47

$4 \mathrm{O} \mathbb{Z}_{2} G$-módulo $\mathcal{F}_{T}(G)$ e Pares de Dualidade de Poincaré $\quad 52$

$4.1 \mathcal{F}_{T}(G)$ e Comensurabilidade . . . . . . . . . . . . . . 52

$4.2 \mathcal{F}_{T}(G)$ e $P D^{n}$-pares $(G, \mathcal{S}) \ldots \ldots \ldots \ldots \ldots \ldots$

5 Decomposição de Grupos e Invariantes $\quad 62$

5.1 Decomposição de Grupos . . . . . . . . . . . . . . . . . . . . 62

5.2 Invariantes $e(G)$ e $e(G, S) \ldots \ldots \ldots \ldots \ldots$

6 Obstruções e Invariantes Cohomológicos $\quad 67$

6.1 Obstrução sing . . . . . . . . . . . . . . . . . . . . 68

6.1.1 A Obstrução sing e os Invariantes $\tilde{e}(G, S)$ e $\tilde{E}(G, S) \ldots \ldots$. . . 70

6.2 A Obstrução sing Generalizada . . . . . . . . . . . . . . . . . . 72

6.3 O Invariante $E\left(G, \mathcal{S}, \mathcal{F}_{T}(G)\right) \ldots \ldots \ldots \ldots$ 
Referências Bibliográficas

Índice Remissivo

88 


\section{INTRODUÇÃO}

Um grupo $G$ se decompõe sobre um subgrupo $S$ se $G=H *_{S} K$ (produto livre com subgrupo amalgamado $S$ ) com $H \neq S \neq K$ ou $G=H *_{S, \sigma}$, (HNN-grupo). São exemplos de grupos que se decompõem $\mathbb{Z}=\{1\} *_{\{1\}}$, id, $\mathbb{Z} * \mathbb{Z}=\mathbb{Z} *_{\{1\}} \mathbb{Z}, \mathbb{Z} \oplus \mathbb{Z}=\mathbb{Z} * \mathbb{Z}$, id .

Uma pergunta interessante é: Quando um grupo se decompõe sobre um subgrupo? Em geral é muito difícil decidir se um grupo $G$ se decompõe sobre um subgrupo $S$ dado, ou mesmo se se decompõem sobre qualquer outro subgrupo. Grupos que se decompõem surgem naturalmente, por exemplo, quando calculamos o grupo fundamental de superfícies compactas, e mais geralmente, de certos CW-complexos ([11]). Vários resultados e pesquisas no sentido de obter classes de grupos que se decompõem sobre um subgrupo tem sido obtidos/desenvolvidas. Há uma forte relação entre decomposição de grupos, teoria de grafos e teoria de ends de grupos e pares de grupos (invariantes cohomológicos). O primeiro resultado de decomposição de grupos envolvendo a teoria clássica de ends de um grupo foi dado por Stallings ([26]). É um resultado sobre decomposição de um grupo sobre um subgrupo finito. Um grupo $G$ é um grupo de dualidade de Poincaré $n$-dimensional sobre $\mathbb{Z}_{2}$ ou, simplesmente, um $P D^{n}$ - grupo se $H^{i}(G ; M) \simeq H_{n-i}(G ; M)$, para todo inteiro $i$, e todo $\mathbb{Z}_{2} G$-módulo $M$. Mais geralmente, um par grupo $(G, \mathcal{S})$, isto é, um par onde $G$ é um grupo e $\mathcal{S}=\left(S_{i}\right)_{i \in I}$ é uma família de subgrupos de $G$, é um par de dualidade de Poincaré $n$-dimensional ou um $P D^{n}$-par ( sobre $\left.\mathbb{Z}_{2}\right)$ se $H^{i}(G ; M) \simeq H_{n-i}(G, \mathcal{S} ; M)$ e $H^{i}(G, \mathcal{S} ; M) \simeq H_{n-i}(G ; M)$ para todo inteiro $i \geq 1$, e todo $\mathbb{Z}_{2} G$-módulo $M$. Considerando $G$ um $P D^{n}$-grupo e $S$ um $P D^{(n-1)}$ - subgrupo de $G$, Kropholler e Roller em [16] analisaram quando $G$ se decompõe sobre um subgrupo $L$ que é comensurável com $S$. Para tanto um $\mathbb{Z}_{2} G$-módulo foi bastante importante, o $\mathbb{Z}_{2} G$-módulo $\mathcal{F}_{S}(G)$ dos subconjuntos $S$-finitos de $G$, mais precisamente, $\mathcal{F}_{S}(G)=\{B \subseteq G$; existe um subconjunto finito $F$ de $G$ com $B \subseteq F . S\}$. De fato, supondo $G$ e $S$ finitamente gerados e que o grupo de cohomologia $H^{1}\left(G ; \mathcal{F}_{S}(G)\right) \simeq \mathbb{Z}_{2}$, os autores provaram que a existência de uma decomposição de $G$, nas hipóteses mencionadas, depende do anulamento de um elemento (obstrução) 
denotado por $\operatorname{sing}_{G}(S)$, ou seja, que uma condição necessária e (sob algumas hipóteses) suficiente, para que $G$ admita uma decomposição sobre um subgrupo comensurável com $S$ é que a imagem do único elemento não nulo de $H^{1}\left(G ; \mathcal{F}_{S} G\right)$ pela aplicação restrição $\operatorname{res}_{S}^{G}: H^{1}\left(G ; \mathcal{F}_{S}(G)\right) \rightarrow H^{1}\left(S ; \mathcal{F}_{S}(G)\right)$ (denotada por $\left.\operatorname{sing}_{G}(S)\right)$ seja nula.

Baseados nos resultados de Kropholler e Roller [16], particularmente relacionados à obstrução $\operatorname{sing}_{G}(S)$ definida pelos autores, Andrade e Fanti obtiveram alguns resultados sobre decomposição de grupos e o invariante $\tilde{E}(G, S)$, quando $G$ e $S$ satisfazem certas condições de dualidade ([1]). De fato, sob as mesmas hipóteses de dualidade em [16], a existência de decomposição é equivalente a $\tilde{E}(G, S)=2$. Trabalhando com par grupo $(G, \mathcal{S})$, onde $\mathcal{S}=\left(S_{i}\right)_{i \in I}$ é uma família de subgrupos de $G$, e hipóteses de dualidade, Kropholler e Roller em [17] apresentaram alguns resultados sobre decomposição de grupos para pares de grupos $(G, \mathcal{S})$, dentre eles, um que estende o resultado anteriormente citado. Para tanto usaram uma obstrução " $\operatorname{sing}_{(G, \mathcal{S})}(T)$ ", que é uma extensão da obstrução $\operatorname{sing}_{G}(S)$ (se consideramos a família vazia $\mathcal{S}=\emptyset$, de subgrupos de $G$ ). Mais precisamente, provaram o seguinte resultado:

([17] Teorema A) Sejam $(G, \mathcal{S})$ um $P D^{n}$-par, $T$ um subgrupo de $G$ que é um $P D^{(n-1)_{-}}$ grupo, e $T$ um subgrupo de $G$ que não é comensurável com nenhum conjugado de $S$, para todo $S$ em $\mathcal{S}$. Então $G$ admite uma decomposição "adaptada" a $\mathcal{S}$, sobre um subgrupo comensurável com $T$ se, e somente se, $\operatorname{sing}_{(G, \mathcal{S})}(T)=0$.

O objetivo de nosso trabalho é estudar alguns dos resultados de Kropholler-Roller, relativos a decomposição de grupos de dualidade de Poincaré. Em especial definir a obstrução "sing generalizada" para pares (uma das principais ferramentas utilizadas nos resultados de Kropholler-Roller), e apresentar alguns resultados de [17] relacionados a tais obstruções. Finalizamos com um resultado para o invariante $E\left(G, \mathcal{S}, \mathcal{F}_{T}(G)\right)$, definido de uma forma mais geral em [2].

Vale destacar que no estudo da sing é importante estudar melhor o homomorfismo "restrição" $\operatorname{res}_{G}^{(G, \mathcal{S})}: H^{1}\left(G, \mathcal{S}, \mathcal{F}_{T}(G)\right) \rightarrow H^{1}\left(G, \mathcal{F}_{T}(G)\right)$ e consequentemente a sequência exata longa em cohomologia para o $\operatorname{par}(G, \mathcal{S})$ e o homomorfismo conexão.

Descrevemos a seguir, de maneira breve, os tópicos principais de cada capítulo.

No capítulo 1, apresentamos alguns conceitos e resultados básicos de Álgebra Homológica. Para maiores detalhes vide [15] e [22].

No capítulo 2 definimos (co)homologia absoluta e relativa. Para (co)homologia absoluta utilizamos [11]. Enquanto que para (co)homologia relativa, a principal referência é [10]. Um importante resultado demonstrado nesse capítulo, e que foi utilizado na prova de resultados apresentados posteriormente, é o da sequência exata longa em cohomologia (Teorema 2.3.1).

No capítulo 3 definimos grupos e pares de dualidade ( $n$-dimensional), em especial grupos e pares de dualidade de Poincaré, também referidos como $P D^{n}$-grupos e $P D^{n_{-}}$ pares, respectivamente. Pares de dualidade de Poincaré de dimensão 2 podem ser obtidos, 
por exemplo, se consideramos $G=\pi_{1}(X)$, onde $X$ é uma superfície fechada orientada de genus $\geq 1$ das quais um número finito $\mathrm{m}$ de discos abertos são removidos e $S=\pi_{1}(\partial X)$.

Como já observamos, no estudo das obstruções sing o $\mathbb{Z}_{2} G$-módulo $\mathcal{F}_{T}(G)$ desempenha um papel fundamental assim, no capítulo 4, várias propriedades envolvendo tal módulo são apresentadas, dentre elas, que $\mathcal{F}_{T}(G)$ é um módulo induzido (Lema 4.1.1), que independe da classe de comensurabilidade de $S$, mais precisamente, que para dois subgrupos $S$ e $T$ de $G, \mathcal{F}_{T}(G)=\mathcal{F}_{S}(G)$ se, e somente se $S$ e $T$ são subgrupos comensuráveis (Proposição 4.1.3). Finalizamos o capítulo com uma interessante caracterização de $P D^{n}$-pares (Teorema 4.2.2).

No capítulo seguinte apresentamos o conceito de decomposição de grupos, alguns exemplos, e resultados envolvendo os invariantes $e(G)$ e $e(G, S)$ estudados em [25] e [24].

Finalmente, no capítulo 6, definimos as obstruções sing utilizadas em [16] e [17] no estudo de decomposição de grupos e/ou pares de dualidade de Poincaré. Um dos principais resultados demonstrados é a Proposição 6.2.3 que é utilizada pelos autores na prova do Teorema A sobre decomposição de um grupo $G$ adaptada a uma família $\mathcal{S}$. Além disso, os invariantes $\widetilde{E}(G, S)$ e $E\left(G, \mathcal{S}, \mathcal{F}_{S}(G)\right)$ bem como alguns resultados envolvendo tais invariantes, decomposição de grupos e/ou dualidade são apresentados ([1], [6]). 


\section{CAPÍTULO 1}

\section{PRELIMINARES}

Apresentamos aqui alguns tópicos de Álgebra Homológica que são úteis na definição de (co)homologia de grupos e verificação de certas propriedades/resultados. Em particular, apresentamos o anel grupo $R G$ e o $\mathbb{Z}_{2} G$-módulo $\mathcal{P}(G)$ do conjunto das partes de $G$, com $G$ um grupo qualquer. Para maiores detalhes sugerimos [15] e [22]. Observamos que em [15], o tratamento apresentado é sobre $R$-módulo com $R$ anel "comutativo", e o anel $R=R G$ não é comutativo se $G$ não é um grupo abeliano (comutativo). No entanto, em geral resultado similares também são válidos quando $R$ é não comutativo. Já [22] trabalha com $R$-módulo para $R$ anel não necessariamente comutativo.

\subsection{Complexos de Cadeias e Cocadeias}

Definição 1.1.1. Seja $R$ um anel com unidade (não necessariamente comutativo). Uma sequência finita ou infinita

$$
\cdots \rightarrow X \stackrel{f}{\rightarrow} Y \stackrel{g}{\rightarrow} Z \rightarrow \cdots
$$

de homomorfismos de R-módulos é dita semi-exata se, e somente se, em cada módulo (exceto nos extremos), a imagem do homomorfismo de entrada está contida no kernel do homomorfismo de saída, e é dita exata se, e somente se, em cada módulo (exceto nos extremos), a imagem do homomorfismo de entrada for igual a o kernel do homomorfismo de saída. 
Observação 1.1.1. 1. Uma tal sequência é semi-exata se, e somente se, a composição $g \circ f$ de quaisquer dois homomorfismos $f$ e g na sequência é o homomorfismo trivial, isto é, $g \circ f=0$.

2. Todo sequência exata de homomorfismos de $R$-módulos é semi-exata, mas nem toda sequência semi-exata é exata.

Os módulos de uma sequência semi-exata C são usualmente indexados por inteiros na ordem decrescente ou por inteiros na ordem crescente.

Se são usados inteiros na ordem decrescente como índices, a sequência semi-exata C é chamada complexo de cadeias (ou sequências baixas) e os homomorfismos em C são todos denotados pelo mesmo símbolo $\partial$. Deste modo, um complexo de cadeias $\mathrm{C}$ é da seguinte forma:

$$
C: \cdots \stackrel{\partial_{n+2}}{\longrightarrow} C_{n+1} \stackrel{\partial_{n+1}}{\longrightarrow} C_{n} \stackrel{\partial_{n}}{\longrightarrow} C_{n-1} \stackrel{\partial_{n-1}}{\longrightarrow} \cdots
$$

com $\partial_{n} \circ \partial_{n+1}=0$.

Neste caso, os elementos $C_{n}$ são chamados cadeias n-dimensionais de C e os homomorfismos $\partial$ são chamados operadores bordo.

O kernel de $\partial_{n}$ é denotado por $Z_{n}(C)$ e é chamado módulo n-dimensional dos ciclos de C. A imagem de $\partial_{n+1}$ é denotada por $B_{n}(C)$ e é chamada módulo n-dimensional dos bordos de C.

O módulo quociente $\frac{Z_{n}(C)}{B_{n}(C)}=\frac{\operatorname{Ker}\left(\partial_{n}\right)}{\operatorname{Im}\left(\partial_{n+1}\right)}$, denotado por $H_{n}(C)$, é chamado módulo de homologia n-dimensional de $\mathrm{C}$ e a coleção $H_{*}(C)=\left\{H_{n}(C)\right\}$ é chamada homologia do complexo de cadeias C.

Quando inteiros na ordem crescente são usados como índices, a sequência semi-exata é chamada complexo de cocadeias (ou sequência superior) e os homomorfismos em $\mathrm{C}$ são usualmente denotados pelo símbolo $\delta$. Deste modo, um complexo de cocadeias $\mathrm{C}$ é da seguinte forma:

$$
C: \cdots \stackrel{\delta^{n-2}}{\longrightarrow} C^{n-1} \stackrel{\delta^{n-1}}{\longrightarrow} C^{n} \stackrel{\delta^{n}}{\longrightarrow} C^{n+1} \stackrel{\delta^{n+1}}{\longrightarrow} \cdots
$$

$\operatorname{com} \delta^{n} \circ \delta^{n-1}=0$.

Neste caso, os termos cocadeia, cociclo e cobordo são usados no lugar de cadeia, ciclo e bordo dos complexos de cadeias. E também, sobrescritos são usados ao invés de subscritos. Finalmente, o módulo quociente $\frac{Z^{n}(C)}{B^{n}(C)}=\frac{\operatorname{Ker}\left(\delta^{n}\right)}{\operatorname{Im}\left(\delta^{n-1}\right)}$, denotado por $H^{n}(C)$, é chamado módulo de cohomologia n-dimensional de $\mathrm{C}$ e a coleção $H^{*}(C)=\left\{H^{n}(C)\right\}$ é chamada cohomologia do complexo de cocadeias $\mathrm{C}$.

Nos dedicaremos aqui aos complexos de cocadeias pois esses são objetos de maior interesse em nossos estudos. 
Observação 1.1.2. Chamamos de complexo de cocadeias trivial o complexo de cocadeias 0 tal que, para todo $n \in \mathbb{Z}, 0^{n}=\{0\} \equiv 0$ (módulo trivial). Todo complexo de cocadeias trivial é exato e temos que $H^{n}(0)=\{0\}$, para todo $n \in \mathbb{Z}$.

Consideremos quaisquer dois complexos de cocadeias de $R$-módulos:

$$
\begin{aligned}
& C: \cdots \stackrel{\delta^{n-2}}{\longrightarrow} C^{n-1} \stackrel{\delta^{n-1}}{\longrightarrow} C^{n} \stackrel{\delta^{n}}{\longrightarrow} C^{n+1} \stackrel{\delta^{n+1}}{\longrightarrow} \cdots \\
& D: \cdots \stackrel{\tilde{\delta}^{n-2}}{\longrightarrow} D^{n-1} \stackrel{\tilde{\delta}^{n-1}}{\longrightarrow} D^{n} \stackrel{\tilde{\delta}^{n}}{\longrightarrow} D^{n+1} \stackrel{\tilde{\delta}^{n+1}}{\longrightarrow} \cdots
\end{aligned}
$$

Por simplicidade denotaremos, em geral, tanto as aplicações de $C^{k}$ em $C^{k+1}$, bem como as aplicações de $D^{k}$ em $D^{k+1}$, simplesmente por $\delta^{k}$.

Definição 1.1.2. Uma aplicação de cocadeias (ou homomorfismos entre complexos de cocadeias) $f: C \rightarrow D$ é uma família de homomorfismos $f=\left\{f^{n}: C^{n} \rightarrow D^{n} ; n \in \mathbb{Z}\right\}$ de $R$-módulos, indexada por inteiros $n \in \mathbb{Z}$, tais que a relação de comutatividade $\delta^{n} \circ f^{n}=$ $f^{n+1} \circ \delta^{n}$ (que às vezes referimos simplesmente por dizer que $\delta \circ f^{n}=f^{n+1} \circ \delta$ ) é verdadeira no retângulo I abaixo:

$$
\begin{gathered}
\cdots \stackrel{\delta^{n-1}}{\longrightarrow} C^{n} \stackrel{\delta^{n}}{\longrightarrow} C^{n+1} \stackrel{\delta^{n+1}}{\longrightarrow} \cdots \\
\downarrow_{f^{n}} I \quad \downarrow_{f^{n+1}} \\
\cdots \stackrel{\delta^{n-1}}{\longrightarrow} D^{n} \stackrel{\delta^{n}}{\longrightarrow} D^{n+1} \stackrel{\delta^{n+1}}{\longrightarrow} \cdots
\end{gathered}
$$

para todo inteiro $n \in \mathbb{Z}$.

Proposição 1.1.1. Seja uma aplicação de cocadeias arbitrária $f: C \rightarrow D$. O homomorfismo $f^{n}: C^{n} \rightarrow D^{n}$ leva $Z^{n}(C)$ em $Z^{n}(D)$ e $B^{n}(C)$ em $B^{n}(D)$.

Demonstração: Provemos que $f^{n}\left(Z^{n}(C)\right) \subset Z^{n}(D)$ e $f^{n}\left(B^{n}(C)\right) \subset B^{n}(D)$.

Seja $f^{n}(x) \in f^{n}\left(Z^{n}(C)\right) \operatorname{com} x \in Z^{n}(C)=\operatorname{Ker}\left(\delta^{n}\right)$, ou seja, $\delta^{n}(x)=0$. Daí, $\delta^{n}\left(f^{n}(x)\right)=f^{n+1}\left(\delta^{n}(x)\right)=f^{n+1}(0)=0$. Portanto, $f^{n}(x) \in \operatorname{Ker}\left(\delta^{n}\right)=Z^{n}(D)$.

Agora, sejam $B^{n}(D)=\left\{\delta^{n-1}(a) ; a \in D^{n-1}\right\}, B^{n}(C)=\left\{\delta^{n-1}(b) ; b \in C^{n-1}\right\}$ e $u \in$ $f^{n}\left(B^{n}(C)\right)$, Então, $u=f^{n}(v)$ tal que $v \in B^{n}(C)$, isto é $v=\delta^{n-1}(b)$, com $b \in C^{n-1}$. Logo, $u=f^{n}(v)=f^{n}\left(\delta^{n-1}(b)\right)=\left(f^{n} \circ \delta^{n-1}\right)(b)=\left(\delta^{n-1} \circ f^{n-1}\right)(b)=\delta^{n-1}\left(f^{n-1}(b)\right) \in \operatorname{Im}\left(\delta^{n-1}\right)$. Portanto, $u \in B^{n}(D)$.

Definição 1.1.3. (Homomorfismo induzido em cohomologia) Seja $f: C \rightarrow D$ uma aplicação de cocadeias. Segue da proposição anterior que, para cada $n \in \mathbb{Z}, f^{n}$ induz uma aplicação bem definida $H^{n}(f): H^{n}(C) \rightarrow H^{n}(D)$ tal que a cada $\bar{x}=x+B^{n}(C)$ 
associa $H^{n}(f)(\bar{x}):=f^{n}(x)+B^{n}(D)$ que é um homomorfismo. Este homomorfismo $H^{n}(f)$ será referido como homomorfismo induzido n-dimensional de f e é também denotado, às vezes, por $f^{*, n}$ ou simplesmente $f^{*}$ sem especificar o nivel $n$.

Observação 1.1.3. Um elemento $x+B^{n}(C) \in H^{n}(D)$ poderá também ser denotado por $[x]$.

Definição 1.1.4. O homomorfismo identidade $i d: C \rightarrow C$ de um complexo de cocadeias $C$,

$$
C: \cdots \stackrel{\delta^{n-2}}{\longrightarrow} C^{n-1} \stackrel{\delta^{n-1}}{\longrightarrow} C^{n} \stackrel{\delta^{n}}{\longrightarrow} C^{n+1} \stackrel{\delta^{n+1}}{\longrightarrow} \cdots
$$

é a família $i d=\left\{i d^{n}: C^{n} \rightarrow C^{n}, n \in \mathbb{Z}\right\}$ de homomorfismos identidade id ${ }^{n}$ dos módulos $C^{n}$, isto é, $i d^{n}(x)=x$. Observe que id é uma aplicação de cocadeias, pois $\delta \circ i d^{n}=i d^{n+1} \circ \delta$.

Definição 1.1.5. Um homomorfismo trivial de um complexo de cocadeias $C$ em um complexo de cocadeias D é o homomorfismo $h: C \rightarrow D$ tal que $h^{n}=0$ é o homomorfismo trivial do módulo $C^{n}$ no módulo $D^{n}$ para todo $n \in \mathbb{Z}$. (Note que claramente $h$ é aplicação de cocadeias pois $\left.\delta \circ 0^{n}=0^{n+1} \circ \delta\right)$. Denotaremos $h=0$ o homomorfismo trivial.

Com isso temos que são válidos os seguintes fatos:

1. Se $i d: C \rightarrow C$ então $H^{n}(i d)=i d_{H^{n}(C)}: H^{n}(C) \rightarrow H^{n}(C)$, para todo $n \in \mathbb{Z}$.

2. Se $f: C \rightarrow D$ e $g: D \rightarrow E$ são homomorfismos de complexos de cocadeias, então $H^{n}(g \circ f)=H^{n}(g) \circ H^{n}(f): H^{n}(C) \rightarrow H^{n}(E)$, para todo $n \in \mathbb{Z}$.

3. Se $h=0: C \rightarrow D$ é o homomorfismo trivial de um complexo de cocadeias $\mathrm{C}$ em um complexo de cocadeias D, então $0=H^{n}(h): H^{n}(C) \rightarrow H^{n}(D)$ é o homomorfismo trivial para todo $n \in \mathbb{Z}$.

Consideremos agora uma sequência exata curta $\mathrm{S}$ de complexos de cocadeias

$$
0 \rightarrow C \stackrel{f}{\rightarrow} D \stackrel{g}{\rightarrow} E \rightarrow 0
$$

isto é, $f: C \rightarrow D$ e $g: D \rightarrow E$ são homomorfismos tais que, para todo $n \in \mathbb{Z}$,

$$
0 \rightarrow C^{n} \stackrel{f^{n}}{\rightarrow} D^{n} \stackrel{g^{n}}{\rightarrow} E^{n} \rightarrow 0
$$

é uma sequência exata curta de $R$-módulos.

A partir de $(\mathrm{S})$ queremos construir uma sequência exata longa envolvendo os módulos de cohomologia $H^{k}(C), H^{k}(D)$ e $H^{k}(E)$ : 


$$
\cdots \rightarrow H^{n}(C) \stackrel{H^{n}(f)}{\rightarrow} H^{n}(D) \stackrel{H^{n}(g)}{\rightarrow} H^{n}(E) \stackrel{\rho^{n}}{\rightarrow} H^{n+1}(C) \stackrel{H^{n+1}(f)}{\rightarrow} \cdots
$$

Os homomorfismos $H^{n}(f)$ e $H^{n}(g)$ já estão definidos, falta exibir os homomorfismos $\rho^{n}$, o que será feito mais adiante, e consequentemente provar que (1) é exata quando (S) é exata. Para este fim, temos o seguinte resultado:

Lema 1.1.1. Se a sequência de complexos (e aplicações) de cocadeias

$$
0 \rightarrow C \stackrel{f}{\rightarrow} D \stackrel{g}{\rightarrow} E \rightarrow 0
$$

é exata, então para todo inteiro n, a sequência

$$
H^{n}(C) \stackrel{H^{n}(f)}{\rightarrow} H^{n}(D) \stackrel{H^{n}(g)}{\rightarrow} H^{n}(E)
$$

de R-módulos é exata.

Demonstração: Como $g \circ f=0$ segue que $H^{n}(g) \circ H^{n}(f)=H^{n}(g \circ f)=0$, para todo $n \in \mathbb{Z}$ e portanto $\operatorname{Im}\left(H^{n}(f)\right) \subset \operatorname{Ker}\left(H^{n}(g)\right)$.

Resta mostrarmos que $\operatorname{Ker}\left(H^{n}(g)\right) \subset \operatorname{Im}\left(H^{n}(f)\right)$, para todo $n \in \mathbb{Z}$.

Seja $\alpha \in \operatorname{Ker}\left(H^{n}(g)\right) \subset H^{n}(D)$. Escolha um cociclo z tal que $\alpha=z+B^{n}(D)$. Como $0=\left[H^{n}(g)\right](\alpha)=g^{n}(z)+B^{n}(E)$, temos que $g^{n}(z) \in B^{n}(E)=\operatorname{Im}\left(\delta^{n-1}\right)$. Daí, existe $y \in E^{n-1}$ tal que $\delta^{n-1}(y)=g^{n}(z)$, onde $\delta^{n-1}$ denota o operador cobordo $\delta^{n-1}: E^{n-1} \rightarrow$ $E^{n}$. Sabemos que $g^{n-1}: D^{n-1} \rightarrow E^{n-1}$ é um epimorfismo, logo existe $x \in D^{n-1}$ tal que $g^{n-1}(x)=y$. Então $g^{n}\left(z-\delta^{n-1}(x)\right)=g^{n}(z)-g^{n}\left(\delta^{n-1}(x)\right)=g^{n}(z)-\delta^{n-1}\left(g^{n-1}(x)\right)=$ $g^{n}(z)-\delta^{n-1}(y)=0$. Isto implica que $z-\delta^{n-1}(x) \in \operatorname{Ker}\left(g^{n}\right)=\operatorname{Im}\left(f^{n}\right)$. Daí, existe $w \in C^{n} \operatorname{com} f^{n}(w)=z-\delta^{n-1}(x)$. Então, $f^{n+1}\left(\delta^{n}(w)\right)=\delta^{n}\left(f^{n}(w)\right)=\delta^{n}\left(z-\delta^{n-1}(x)\right)=$ $\delta^{n}(z)-\left(\delta^{n} \circ \delta^{n-1}\right)(x)=0$, pois z é um cociclo e $\delta^{n} \circ \delta^{n-1}=0$.

Como $f^{n+1}$ é um monomorfismo, segue que $\delta^{n}(w)=0$ e assim, $w \in Z^{n}(C)$. Seja $\beta=w+B^{n}(C) \in H^{n}(C)$. Desde que $z \in Z^{n}(D)$ e $\delta^{n-1}(x) \in B^{n}(D)$, temos que $f^{n}(w)+B^{n}(D)=\left(z-\delta^{n-1}(x)\right)+B^{n}(D)=z+B^{n}(D)$. Logo, $\left(H^{n}(f)\right)(\beta)=f^{n}(w)+$ $B^{n}(D)=z+B^{n}(D)=\alpha$ e assim, $\operatorname{Ker}\left(H^{n}(g)\right) \subset \operatorname{Im}\left(H^{n}(f)\right)$.

Portanto, a sequência é exata.

Agora, vamos construir o homomorfismo conexão $\rho^{n}$ já citado anteriormente.

Construção de $\rho^{n}: H^{n}(E) \rightarrow H^{n+1}(C)$ (homomorfismo conexão):

Seja $\bar{z} \in H^{n}(E), z \in Z^{n}(E) \subset E^{n}$ e considere a seguinte representação: 


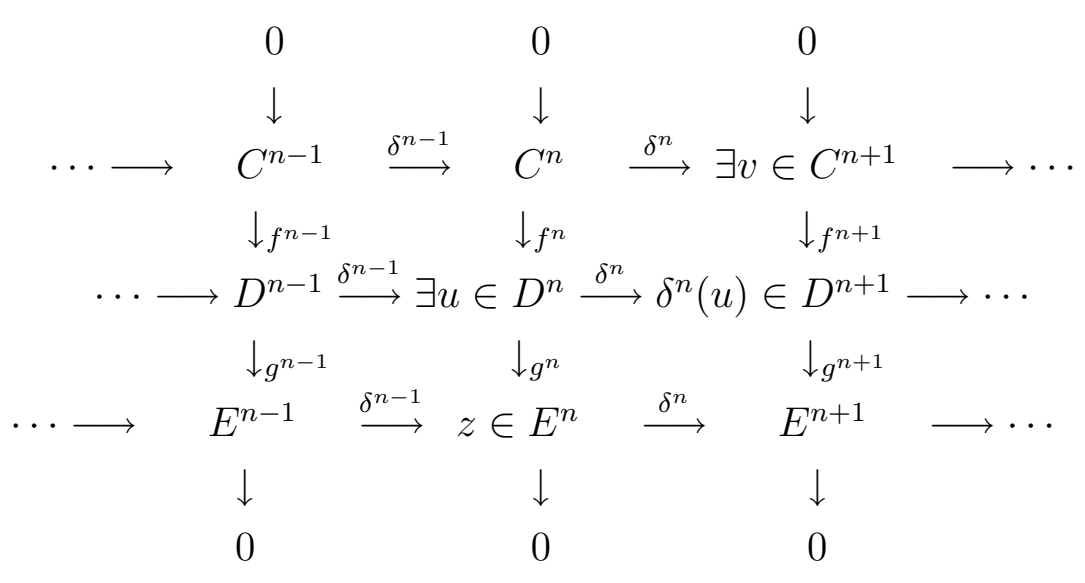

Passos:

1. Define-se uma aplicação $\phi: Z^{n}(E) \rightarrow H^{n+1}(C)$.

Dado $[z] \in H^{n}(E), z \in Z^{n}(E) \subset E^{n}$, como $g^{n}$ é sobrejetora, existe $\underline{u \in D^{n}}$ tal que $g^{n}(u)=z$.

- Temos que $\delta^{n}(u) \in \operatorname{Ker}\left(g^{n+1}\right)=\operatorname{Im}\left(f^{n+1}\right)$, pois $g^{n+1}\left[\delta^{n}(u)\right]=\delta^{n}\left[g^{n}(u)\right]=$ $\delta^{n}(z)=0$.

- Agora, como $\delta^{n}(u) \in \operatorname{Im}\left(f^{n+1}\right)$ e $f^{n+1}$ é injetora, então existe um único $\underline{v \in C^{n+1}}$ tal que $\underline{f^{n+1}(v)=\delta^{n}(u)}$.

- Afirmação: se $g^{n}(u)=z$ e $g^{n}\left(u^{\prime}\right)=z, f^{n+1}(v)=\delta^{n}(u)$ e $f^{n+1}\left(v^{\prime}\right)=\delta^{n}\left(u^{\prime}\right)$, então $v-v^{\prime} \in B^{n+1}(C)$, e portanto $v+B^{n+1}(C)=v^{\prime}+B^{n+1}(C)$.

De fato, consideremos $u, u^{\prime}$ e $v, v^{\prime}$ como acima. Como $g^{n}\left(u-u^{\prime}\right)=g^{n}(u)-$ $g^{n}\left(u^{\prime}\right)=z-z=0$, temos que $u-u^{\prime} \in \operatorname{Ker}\left(g^{n}\right)=\operatorname{Im}\left(f^{n}\right)$. Daí, existe $y \in C^{n}$ $\operatorname{com} f^{n}(y)=u-u^{\prime}$. Então, $f^{n+1}\left(v-v^{\prime}-\delta^{n}(y)\right)=f^{n+1}(v)-f^{n+1}\left(v^{\prime}\right)-$ $f^{n+1}\left(\delta^{n}(y)\right)=\delta^{n}(u)-\delta^{n}\left(u^{\prime}\right)-\delta^{n}\left(f^{n}(y)\right)=\delta^{n}(u)-\delta^{n}\left(u^{\prime}\right)-\delta^{n}\left(u-u^{\prime}\right)=0$. Visto que $f^{n+1}$ é um monomorfismo, segue que $v-v^{\prime}=\delta^{n}(y) \in B^{n+1}(C)$. Logo, $v+B^{n+1}(C)=v^{\prime}+B^{n+1}(C)$.

- Assim, fica bem definida uma aplicação $\phi: Z^{n}(E) \rightarrow H^{n+1}(C)$, que associa $z \in Z^{n}(E)$ a $v+B^{n+1}(C)=\bar{v}$ e $\phi$ independe do $u$ inicialmente escolhido.

2. Mostra-se que $\phi$ é homomorfismo.

Sejam $z, z^{\prime} \in Z^{n}(E)$ e $\alpha, \alpha^{\prime} \in A$. Escolha $u, u^{\prime} \in D^{n}$ e $v, v^{\prime} \in C^{n+1}$ satisfazendo $g^{n}(u)=z, g^{n}\left(u^{\prime}\right)=z^{\prime}$ e $f^{n+1}(v)=\delta^{n}(u), f^{n+1}\left(v^{\prime}\right)=\delta^{n}\left(u^{\prime}\right)$. Então, $g^{n}\left(\alpha u+\alpha^{\prime} u^{\prime}\right)=$ $\alpha z+\alpha^{\prime} z^{\prime}, f^{n+1}\left(\alpha v+\alpha^{\prime} v^{\prime}\right)=\delta^{n}\left(\alpha u+\alpha^{\prime} u^{\prime}\right)$. Isto implica que $\phi\left(\alpha z+\alpha^{\prime} z^{\prime}\right)=(\alpha v+$ $\left.\alpha^{\prime} v^{\prime}\right)+B^{n+1}(C)=\alpha v+B^{n+1}(C)+\alpha^{\prime} v^{\prime}+B^{n+1}(C)=\alpha \bar{v}+\alpha^{\prime} \bar{v}^{\prime}=\alpha \phi(z)+\alpha^{\prime} \phi\left(z^{\prime}\right)$.

Portanto, $\phi$ é um homomorfismo de módulos. 
3. Mostra-se que $B^{n}(E) \subset K \operatorname{Ker}(\phi)$.

Seja $z \in B^{n}(E)$. Por definição de $B^{n}(E)$, existe $y \in E^{n-1} \operatorname{com} \delta^{n-1}(y)=z$. Como $g^{n-1}$ é epimorfismo, existe $w \in D^{n-1}$ satisfazendo $g^{n-1}(w)=y$.

Seja $u=\delta^{n-1}(w)$. Então, $g^{n}(u)=g^{n}\left(\delta^{n-1}(w)\right)=\delta^{n-1}\left(g^{n-1}(w)\right)=\delta^{n-1}(y)=z$.

Por outro lado, $\delta^{n}(u)=\left(\delta^{n} \circ \delta^{n-1}\right)(w)=0$. Assim, podemos escolher $v=0 \in C^{n+1}$ de modo que satisfaça $f^{n+1}(v)=f^{n+1}(0)=0=\delta^{n}(u)$.

Pela definição de $\phi$, obtemos $\phi(z)=v+B^{n+1}(C)=0+B^{n+1}(C)=\overline{0}$.

Portanto, $z \in \operatorname{Ker}(\phi)$.

4. Finalmente, de 1., 2. e 3., fica bem definido $\rho^{n}: H^{n}(E) \rightarrow H^{n+1}(C)$, que associa a cada $[z] \in H^{n}(E)$ a $\phi([z])=[v] \in H^{n+1}(C)$.

Pois, se $[z]=\left[z^{\prime}\right]$ então $z-z^{\prime} \in B^{n}(E)$. Assim, $\phi\left(z-z^{\prime}\right)=0$ e portanto, $\phi(z)=\phi\left(z^{\prime}\right)$.

Definição 1.1.6. O homomorfismo $\rho^{n}$ acima construído, isto é, $\rho^{n}: H^{n}(E) \rightarrow H^{n+1}(C)$; $[z]=z+B^{n}(E) \mapsto \rho^{n}([z])=[v]=v+B^{n+1}(C)$ (onde $v$ é um elemento determinado de modo que $f^{n+1}(v)=\delta^{n}(u)$ e $\left.g^{n}(u)=z\right)$, é denominado homomorfismo conexão ou homomorfismo conectante.

Agora, de posse desses resultados, estamos aptos a demonstrar o seguinte Teorema.

Teorema 1.1.1. Se a sequência

$$
0 \rightarrow C \stackrel{f}{\rightarrow} D \stackrel{g}{\rightarrow} E \rightarrow 0
$$

é uma sequência exata curta de complexos de cocadeias, então a sequência longa em cohomologia

$$
\cdots \rightarrow H^{n}(C) \stackrel{H^{n}(f)}{\rightarrow} H^{n}(D) \stackrel{H^{n}(g)}{\rightarrow} H^{n}(E) \stackrel{\rho^{n}}{\rightarrow} H^{n+1}(C) \stackrel{H^{n+1}(f)}{\rightarrow} \cdots
$$

é exata.

Demonstração: Pelo Lema 1.1.1, temos que $\operatorname{Im}\left(H^{n}(f)\right)=\operatorname{Ker}\left(H^{n}(g)\right)$, logo, resta provar as seguintes igualdades:

1. $\operatorname{Im}\left(H^{n}(g)\right)=\operatorname{Ker}\left(\rho^{n}\right)$.

2. $\operatorname{Im}\left(\rho^{n}\right)=\operatorname{Ker}\left(H^{n+1}(f)\right)$.

Verifiquemos 1. Estamos interessados na seguinte parte da sequência longa de cohomologia: 


$$
H^{n}(D) \stackrel{H^{n}(g)}{\rightarrow} H^{n}(E) \stackrel{\rho^{n}}{\rightarrow} H^{n+1}(C)
$$

Seja $\alpha \in \operatorname{Im}\left(H^{n}(g)\right) \subset H^{n}(E)$. Então existe $y \in Z^{n}(D)$ tal que $\alpha=g^{n}(y)+B^{n}(E)$. Seja $z=g^{n}(y)$ com $y \in Z^{n}(D)$, isto é, $\delta^{n}(y)=0$.

Na definição de $\rho^{n}$, podemos escolher $u=y$ e $v=0 \in C^{n+1}$ satisfazendo $f^{n+1}(v)=$ $0=\delta^{n}(u)$. Segue da definição de $\rho^{n}: H^{n}(E) \rightarrow H^{n+1}(C)$ que $\rho^{n}(\alpha)=0+B^{n+1}(C)=[0]$ e daí, $\alpha \in \operatorname{Ker}\left(\rho^{n}\right)$. Logo, $\operatorname{Im}\left(H^{n}(g)\right) \subset \operatorname{Ker}\left(\rho^{n}\right)$.

Agora, seja $\alpha=z+B^{n}(E) \in \operatorname{Ker}\left(\rho^{n}\right) \subset H^{n}(E)$. Pela definição de $\rho^{n}(\alpha)$, existem $u \in D^{n}$ e $v \in C^{n+1}$ satisfazendo $g^{n}(u)=z$ e $f^{n+1}(v)=\delta^{n}(u)$ tais que $\rho^{n}(\alpha)=v+B^{n+1}(C)$. Como $\rho^{n}(\alpha)=0 \in H^{n+1}(C)$, segue que $v \in B^{n+1}(C)$. Daí, existe $w \in C^{n} \operatorname{com} \delta^{n}(w)=v$.

Seja $y=u-f^{n}(w) \in D^{n}$. Então, $\delta^{n}(y)=\delta^{n}\left(u-f^{n}(w)\right)=\delta^{n}(u)-\delta^{n}\left(f^{n}(w)\right)=$ $\delta^{n}(u)-f^{n+1}\left(\delta^{n}(w)\right)=\delta^{n}(u)-f^{n+1}(v)=0$. Isto implica que $y \in Z^{n}(D)$.

Considere $\beta=y+B^{n}(D)$. Como $g^{n}(y)=g^{n}\left(u-f^{n}(w)\right)=g^{n}(u)-g^{n}\left(f^{n}(w)\right)=$ $z-0=z$, temos que $\left(H^{n}(g)\right)(\beta)=g^{n}\left(y+B^{n}(D)\right)=g^{n}(y)+B^{n}(E)=z+B^{n}(E)=\alpha$ e daí, $\alpha \in \operatorname{Im}\left(H^{n}(g)\right)$. Logo, $\operatorname{Ker}\left(\rho^{n}\right) \subset \operatorname{Im}\left(H^{n}(g)\right)$.

Portanto, $\operatorname{Im}\left(H^{n}(g)\right)=\operatorname{Ker}\left(\rho^{n}\right)$.

Verifiquemos agora a segunda igualdade. Nesse caso estamos interessados na seguinte parte da sequência longa de cohomologia

$$
H^{n}(E) \stackrel{\rho^{n}}{\rightarrow} H^{n+1}(C) \stackrel{H^{n+1}(f)}{\rightarrow} H^{n+1}(D) .
$$

Seja $\alpha \in \operatorname{Im}\left(\rho^{n}\right) \subset H^{n+1}(C)$. Então

existe $\beta \in H^{n}(E)$ com $\rho^{n}(\beta)=\alpha$. Escolha um cociclo $z \in Z^{n}(E)$ tal que $\beta=$ $z+B^{n}(E)$. Por definição de $\rho^{n}$ segue que existem $u \in D^{n}$ e $v \in C^{n+1}$ satisfazendo $g^{n}(u)=z$ e $f^{n+1}(v)=\delta^{n}(u) \in B^{n+1}(D)$ e $\alpha=\rho^{n}(\beta)=\rho^{n}\left(z+B^{n}(E)\right)=v+B^{n+1}(C)$. Daí, $\left(H^{n+1}(f)\right)(\alpha)=f^{n+1}(v)+B^{n+1}(D)=[0]$, pois $f^{n+1}(v) \in B^{n+1}(D)$ e assim, $\alpha \in$ $\operatorname{Ker}\left(H^{n+1}(f)\right)$. Logo, $\operatorname{Im}\left(\rho^{n}\right) \subset \operatorname{Ker}\left(H^{n+1}(f)\right)$.

Seja agora $\alpha \in \operatorname{Ker}\left(H^{n+1}(f)\right) \subset H^{n+1}(C)$. Escolha um cociclo $z \in Z^{n+1}(C)$ tal que $\alpha=z+B^{n+1}(C)$. Como $0=\left(H^{n+1}(f)\right)(\alpha)=f^{n+1}(z)+B^{n+1}(D)$ segue que $f^{n+1}(z) \in$ $B^{n+1}(D)$ e daí existe $u \in D^{n}$ satisfazendo $\delta^{n}(u)=f^{n+1}(z)$.

Seja $y=g^{n}(u) \in E^{n}$. Então temos que $\delta^{n}(y)=\delta^{n}\left(g^{n}(u)\right)=g^{n+1}\left(\delta^{n}(u)\right)=g^{n+1}\left(f^{n+1}(z)\right)=$ 0 , pois $g^{n+1} \circ f^{n+1}=0$, visto que a sequência de complexos é exata. Isto implica que $y \in Z^{n}(E)$.

Seja $\beta=y+B^{n}(E) \in H^{n}(E)$. Como $g^{n}(u)=y$ e $f^{n+1}(z)=\delta^{n}(u)$, segue da definição de $\rho^{n}$ que $\rho^{n}(\beta)=\rho^{n}\left(y+B^{n}(E)\right)=z+B^{n+1}(C)=\alpha$ e daí, $\alpha \in \operatorname{Im}\left(\rho^{n}\right)$. Logo, $\operatorname{Ker}\left(H^{n+1}(f)\right) \subset \operatorname{Im}\left(\rho^{n}\right)$.

Portanto, $\operatorname{Im}\left(\rho^{n}\right)=\operatorname{Ker}\left(H^{n+1}(f)\right)$.

Corolário 1.1.1. Se dois dos três complexos de cocadeias $C, D$ e E na sequência exata curta 


$$
0 \rightarrow C \stackrel{f}{\rightarrow} D \stackrel{g}{\rightarrow} E \rightarrow 0
$$

são exatos, então o complexo de cocadeias remanescente também o é.

Corolário 1.1.2. Se no seguinte diagrama comutativo de homomorfismos de R-módulos todas as três linhas são exatas e todas as três colunas são semi-exatas, então a exatidão de quaisquer duas colunas implica a exatidão da coluna restante.

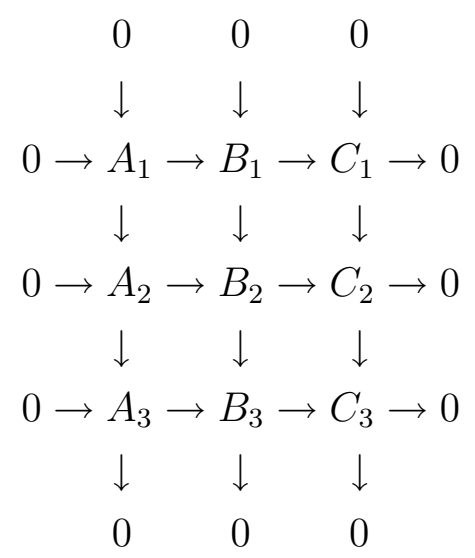

Observação 1.1.4. Similarmente define-se aplicação de cadeias e prova-se a existência de uma sequência exata longa em homologia associada a uma sequência exata curta

$$
0 \rightarrow C \stackrel{f}{\rightarrow} D \stackrel{g}{\rightarrow} E \rightarrow 0
$$

de complexos de cadeias.

Definição 1.1.7. Dois homomorfismos (ou aplicações de cocadeias) $f, g: C \rightarrow D$ são ditos homotópicos se, e somente se, existe uma familia de homomorfismos ("de grau $(-1) ") h=\left\{h^{n}: C^{n} \rightarrow D^{n-1}, \forall n \in \mathbb{Z}\right\}$ tal que, para todo $n \in \mathbb{Z}, \delta^{n-1} \circ h^{n}+h^{n+1} \circ \delta^{n}=$ $f^{n}-g^{n}: C^{n} \rightarrow D^{n}$ conforme indica o seguinte diagrama:

$$
\begin{aligned}
\cdots & \stackrel{\delta^{n-2}}{\longrightarrow} C^{n-1} \stackrel{\delta^{n-1}}{\longrightarrow} C^{n} \stackrel{\delta^{n}}{\longrightarrow} C^{n+1} \stackrel{\delta^{n+1}}{\longrightarrow} \cdots \\
& \stackrel{f^{n-1} \downarrow h^{n} \swarrow f^{n} \downarrow_{g^{n}} \swarrow h^{n+1} \downarrow_{f^{n+1}}}{\longrightarrow} \stackrel{\delta^{n-2}}{\longrightarrow} D^{n-1} \stackrel{\delta^{n-1}}{\longrightarrow} D^{n} \stackrel{\delta^{n}}{\longrightarrow} D^{n+1} \stackrel{\delta^{n+1}}{\longrightarrow} \cdots
\end{aligned}
$$

Definição 1.1.8. A familia $h$, descrita acima, é chamada uma homotopia (ou uma homotopia de cocadeias) entre a aplicação de cocadeias $f$ e a aplicação g. Em símbolos, $h: f \simeq g: C \rightarrow D$. Dizemos também que a aplicação de cocadeias $f$ é homotópica a $g$ (ou f e g são homotópicas) e escrevemos simplesmente $f \simeq g$ se existe um homotopia de f para $g$. 
Proposição 1.1.2. Se dois homomorfismos $f, g: C \rightarrow D$ de complexos de cocadeias são homotópicos, então $H^{n}(f)=H^{n}(g): H^{n}(C) \rightarrow H^{n}(D)$, para todo inteiro $n \in \mathbb{Z}$.

Demonstração: Por hipótese, existe $h: f \simeq g: C \rightarrow D$, isto é, uma família de homomorfismos $h=\left\{h^{i}: C^{i+1} \rightarrow D^{i}\right\}_{i}$ tal que $\delta^{n-1} \circ h^{n}+h^{n+1} \circ \delta^{n}=f^{n}-g^{n}$.

Provemos que $H^{n}(f)=H^{n}(g)$.

Seja $u \in H^{n}(C)$. Escolha $z \in Z^{n}(C) \subset C^{n}$ tal que $u=[z]=z+B^{n}(C)$. Então $f^{n}(z)-g^{n}(z)=\delta^{n-1}\left(h^{n}(z)\right)+h^{n+1}\left(\delta^{n}(z)\right)=\delta^{n-1}\left(h^{n}(z)\right)$, uma vez que $\delta^{n}(z)=0$. Como $\delta^{n-1}\left(h^{n}(z)\right) \in B^{n}(D)$, temos que $f^{n}(z)+B^{n}(D)=g^{n}(z)+B^{n}(D), \operatorname{assim}\left(H^{n}(f)\right)([z])=$ $f^{n}(z)+B^{n}(D)=g^{n}(z)+B^{n}(D)=\left(H^{n}(g)\right)([z])$ e portanto, $H^{n}(f)=H^{n}(g)$.

Definição 1.1.9. (Equivalência de homotopia e equivalência fraca)

(i) Uma aplicação de cocadeias $f: C \rightarrow D$ é chamada uma equivalência de homotopia se existe um aplicação de cocadeias $g: D \rightarrow C$ tal que $g \circ f \simeq i d_{C}$ e $f \circ g \simeq i d_{D}$. Dizemos que dois complexos de cocadeias $C$ e D são homotopicamente equivalentes se existe uma equivalência de homotopia $f: C \rightarrow D$.

(ii) Uma aplicação de cocadeias $f: C \rightarrow D$ é chamada uma equivalência fraca se $H(f): H(C) \rightarrow H(D)$ é um isomorfismo, isto é, $H^{n}(f): H^{n}(C) \rightarrow H^{n}(D)$ é um isomorfismo, para todo $n \in \mathbb{Z}$.

Proposição 1.1.3. Toda equivalência de homotopia é uma equivalência fraca. Em outras palavras, se $C$ e $D$ são complexos de cocadeias homotopicamente equivalentes então $H^{n}(C) \simeq H^{n}(D)$, para todo $n \in \mathbb{Z}$.

Demonstração: Se $f: C \rightarrow D$ é uma equivalência de homotopia e $g: D \rightarrow C$ é tal que $g \circ f \simeq i d_{C}$ e $f \circ g \simeq i d_{D}$ então dos resultados anteriores, obtemos que $H^{n}(g) \circ H^{n}(f)=$ $H^{n}(g \circ f)=H^{n}\left(i d_{C}\right)=i d_{H^{n}(C)}$ e $H^{n}(f) \circ H^{n}(g)=H^{n}(f \circ g)=H^{n}\left(i d_{D}\right)=i d_{H^{n}(D)}$. Assim, $H^{n}(f)$ é um isomorfismo, para todo $n \in \mathbb{Z}$ e $\left(H^{n}(f)\right)^{-1}=H^{n}(g)$.

Observação 1.1.5. De maneira similar define-se homotopia entre aplicação de cadeias, equivalência de homotopia, equivalência fraca (para aplicações de cadeia) e complexos de cadeia homotopicamente equivalentes.

\subsection{Anel grupo e $\mathcal{R} G$-módulos}

Definição 1.2.1. Sejam $\mathcal{R}$ um anel comutativo com unidade $1, G$ um grupo, denotado multiplicativamente, com elemento neutro $1_{G}$. Seja $\mathcal{R} G$ o $\mathcal{R}$-módulo livre gerado pelos 
elementos de $G$. Deste modo um elemento de $\mathcal{R} G$ é expresso por somas $\sum_{g \in G} r_{g} g$, onde $r_{g} \in \mathcal{R}$ e $r_{g}=0$ para quase todo $g \in G$, exceto num número finito.

Definindo em $\mathcal{R} G$ a multiplicação

$$
\left(\sum_{g \in G} r_{g} g\right) \cdot\left(\sum_{h \in G} s_{h} h\right)=\left(\sum_{g, h \in G} r_{g} s_{h} g h\right)
$$

e a soma

$$
\left(\sum_{g \in G} r_{g} g\right)+\left(\sum_{h \in G} s_{g} g\right)=\sum_{g \in G}\left(r_{g}+s_{g}\right) g .
$$

teremos uma estrutura de anel, o qual tem unidade $1_{\mathcal{R} G}=1.1_{G}$ e é chamado anel grupo de $G$ sobre $\mathcal{R}$.

Observação 1.2.1. 1. Se $\mathcal{R}=\mathbb{Z}_{2}$ então um elemento $x \in \mathbb{Z}_{2} G$ é da seguinte forma

$$
x=\overline{1} g_{1}+\cdots+\overline{1} g_{k},
$$

onde $\overline{1} \in \mathbb{Z}_{2}$ e $g_{i} \in G, i=1, \ldots, k$.

Às vezes tal elemento é denotado simplesmente por

$$
x=1 g_{1}+\cdots+1 g_{k} \text {, ou ainda, } x=g_{1}+\cdots+g_{k} .
$$

2. $O$ anel $\mathcal{R} G$ pode não ser comutativo. Isso acontece sempre que $G$ é um grupo não comutativo.

Definição 1.2.2. Para qualquer grupo $G$ o homomorfismo $\epsilon: \mathcal{R} G \rightarrow \mathcal{R}$ tal que $\epsilon(g)=1$, para todo $g \in G$ e estendido por linearidade, ou seja, $\epsilon\left(\sum_{g \in G} r_{g} g\right)=\sum_{g \in G} r_{g} \epsilon(g)=\sum_{g \in G} r_{g}$, é denominado aplicação aumentação. O Kernel de $\epsilon$ é chamado de ideal aumentação de $\mathcal{R} G$. Quando $\mathcal{R}=\mathbb{Z}_{2}$ e $\epsilon: \mathbb{Z}_{2} G \rightarrow \mathcal{R}$, denotaremos $K \operatorname{Ker}(\epsilon)$ por $\mathcal{G}$ (é também comum denotar $\operatorname{Ker}(\epsilon)$ por $\Delta$, porém aqui usaremos $\Delta$ para indicar o núcleo de uma outra "aplicação aumentação" que definiremos posteriormente).

Definição 1.2.3. Sejam G um grupo denotado multiplicativamente e $M$ um conjunto não vazio. Uma ação (à esquerda) de $G$ sobre $M$ (ou uma $G$-ação sobre $M$ ) é uma aplicação

$$
\begin{aligned}
\mu: G \times M & \rightarrow M \\
(g, m) & \mapsto \mu(g, m) \stackrel{\text { not. }}{=} g . m
\end{aligned}
$$

satisfazendo: 
(a) $1 . m=m$, para todo $m \in M$,

(b) $\left(g_{1} g_{2}\right) m=g_{1}\left(g_{2} m\right)$, para todos $g_{1}, g_{2} \in G$ e $m \in M$.

Se na definição acima $M$ tiver uma estrutura de grupo aditivo, para que esta estrutura seja preservada pela $G$-ação, além das condições $(a)$ e $(b)$ exige-se ainda a seguinte condição:

(c) $g\left(m_{1}+m_{2}\right)=g m_{1}+g m_{2}$, para $g \in G$ e $m_{1}, m_{2} \in M$.

Definição 1.2.4. Quando um grupo $G$ está atuando em um conjunto $M$ dizemos que o conjunto $M$ é um G-conjunto.

Observação 1.2.2. 1. Podemos definir, de forma equivalente, uma G-ação (à esquerda) sobre $M$ como sendo um homomorfismo

$$
\begin{aligned}
\mu: G & \rightarrow B_{i j}(M) \\
g & \mapsto \mu(g)=\mu_{g} ; \mu_{g}(m)=g \cdot m,
\end{aligned}
$$

onde $B_{i j}(M)=\{f: M \rightarrow M ; f$ é uma bijeção $\}$ é o grupo das bijeções, com relação a operação composição.

2. Analogamente, define-se $G$-ação à direita sobre $M$.

3. Dada uma $G$-ação à esquerda sobre $M$, sempre podemos definir uma $G$-ação à direita sobre $M$ por $m * g:=g^{-1} m$. Assim, todo $\mathbb{Z}_{2} G$-módulo à esquerda $M$ é também um $\mathbb{Z}_{2} G$-módulo à direita. Similarmente, todo $\mathbb{Z}_{2} G$-módulo à direita é um $\mathbb{Z}_{2} G$-módulo $\grave{a}$ esquerda.

Definição 1.2.5. Dizemos que uma ação de $G$ em $M$ é livre se a seguinte condição for verdadeira:

$$
g m=m, \text { para algum } m \in M \text { se, e somente se, } g=1 \text {. }
$$

Definição 1.2.6. Dizemos que uma $G$-ação sobre $M$ é trivial se:

$$
\text { g.m } m \text { m, para todo } m \in M \text { e todo } g \in G \text {. }
$$


Proposição 1.2.1. Seja $G$ um grupo e $M$ um conjunto não vazio. Então, $M$ é um $\mathcal{R} G$ módulo (à esquerda) se, e somente se, $M$ é um $\mathcal{R}$-módulo (à esquerda) munido de uma ação sobre $M$ (à esquerda) de $G$ sobre o grupo aditivo $(M,+)$.

Demonstração: Se $M$ é um $\mathcal{R} G$-módulo então $M$ é um $\mathcal{R}$-módulo considerando $r m:=\left(r 1_{G}\right) m$, e pode-se definir uma $G$-ação por $g \cdot m:=\left(1_{\mathcal{R}} \cdot g\right) m$.

Reciprocamente, se $M$ é um $\mathcal{R}$-módulo e existe uma ação de $G$ sobre $M$ então podemos dar a $M$ uma estrutura de $\mathcal{R} G$-módulo da seguinte maneira $\left(\sum_{g \in G} r_{g} \cdot g\right) m:=\sum_{g \in G} r_{g} \cdot(g \cdot m)$.

Corolário 1.2.1. $M$ é um $\mathbb{Z} G$-módulo se, e somente se, $M$ é um grupo abeliano munido de uma G-ação.

Corolário 1.2.2. $M$ é um $\mathbb{Z}_{2} G$-módulo se, e somente se, $M$ é um $\mathbb{Z}_{2}$-módulo (equivalentemente, $M$ é um grupo abeliano em que todo elemento tem ordem 2) munido de uma $G$-ação.

Segue dos Corolários anteriores que todo $\mathbb{Z}_{2} G$-módulo é um $\mathbb{Z} G$-módulo, mas a recíproca claramente não é verdadeira.

Exemplo 1.2.1. Seja $G$ um grupo. Então $\mathcal{P}(G)$, o conjunto das partes de $G$, é um $\mathbb{Z}_{2} G$-módulo, onde consideraremos $\mathcal{P}(G)$ um grupo abeliano com a operação diferença simétrica, $A+B:=A \triangle B=(A-B) \cup(B-A)=(A \cup B)-(A \cap B)$, e a $G$-ação natural é dada por

$$
\begin{aligned}
G \times \mathcal{P}(G) & \rightarrow \mathcal{P}(G) \\
& (g, H) \mapsto g . H=\{g . x ; x \in H\} .
\end{aligned}
$$

Note que todo elemento de $\mathcal{P}(G)$ tem ordem 2 pois $A+A=\emptyset$.

Observação 1.2.3. Todo $\mathcal{R}$-módulo $M$ pode ser visto como um $\mathcal{R} G$-módulo com a $G$ ação trivial. Neste caso, dizemos que $M$ é um $\mathcal{R} G$-módulo trivial. Para nós, $M=\mathcal{R}$ será, em geral, considerado um $\mathcal{R} G$-módulo trivial e assim,

$\left(\sum_{g \in G} r_{g} \cdot g\right) r:=\sum_{g \in G} r_{g}(g \cdot r)=\sum_{g \in G} r_{g} \cdot r=\epsilon\left(\sum_{g \in G} r_{g} \cdot g\right) r$.

Quando $\mathcal{R}=\mathbb{Z}_{2}$ esta é a única estrutura de $\mathbb{Z}_{2} G$-módulo possivel, pois Aut $\left(\mathbb{Z}_{2}\right)=\{i d\}$.

Definição 1.2.7. Sejam $M$ um $G$-conjunto e $m \in M$.

(i) A G-órbita de $m$, denotada por $G(m)$, é o subconjunto de $M$ dado por: 


$$
G(m):=\{g . m ; g \in G\}
$$

(ii) O estabilizador de $m$ (ou subgrupo de isotropia de $G$ em $m$ ) denotado por $G_{m}$, é o seguinte subgrupo de $G$ :

$$
G_{m}=\{g \in G ; g m=m\}
$$

Pode-se verificar o seguinte resultado:

Proposição 1.2.2. Se $M$ é um $G$-conjunto, então as órbitas de $M$ formam uma partição de $M$. Assim, $M=\bigcup_{m_{i} \in E} G\left(m_{i}\right)$, onde E é um conjunto de representantes para as órbitas distintas.

Considerando $X$ um $G$-conjunto e $\mathcal{R} X$ o $\mathcal{R}$-módulo livre gerado pelos elementos de $X$, podemos estender a $G$-ação sobre $X$ a uma $G$-ação sobre $\mathcal{R} X$ da seguinte maneira: $g .\left(\sum r_{x} x\right):=\sum r_{x}(g \cdot x)$, com $g \in G, r_{x} \in \mathcal{R}$ e $x \in X$. Assim, temos o seguinte resultado:

Proposição 1.2.3. Sejam $X$ um $G$-conjunto livre e $E$ um conjunto de representantes para as G-órbitas em $X$. Então $\mathcal{R} X$ é um $\mathcal{R} G$-módulo livre com base $E$.

Demonstração: Seja $E=\left\{x_{\lambda}: \lambda \in \Lambda\right\}$ um conjunto de representantes para as $G$-órbitas em $X$. Então $X=\bigcup_{x_{\lambda \in E}} G\left(x_{\lambda}\right)$ e assim, $\mathcal{R} X=\mathcal{R}\left(\bigcup_{x_{\lambda \in E}} G\left(x_{\lambda}\right)\right)=\bigoplus_{x_{\lambda} \in E} \mathcal{R}\left(G\left(x_{\lambda}\right)\right)$ (vide [11], p.13).

Agora, como a $G$-ação é livre temos, para cada $x_{\lambda}$, que a aplicação

$$
\begin{aligned}
f_{\lambda}: G & \rightarrow G\left(x_{\lambda}\right) \\
g & \mapsto f_{\lambda}(g)=g \cdot x_{\lambda}
\end{aligned}
$$

é uma bijeção. De fato, $f_{\lambda}$ é claramente sobrejetora, e se tivermos $g_{1}, g_{2} \in G$ tais que $f_{\lambda}\left(g_{1}\right)=f_{\lambda}\left(g_{2}\right)$ então, $g_{1} x_{\lambda}=g_{2} x_{\lambda}$, ou seja, $x_{\lambda}=g_{1}{ }^{-1} g_{2} x_{\lambda}$ e como a G-ação é livre, $g_{1}{ }^{-1} g_{2}=1$ o que leva a $g_{1}=g_{2}$. Logo, $f_{\lambda}$ é injetora.

Daí, $\mathcal{R}\left(G\left(x_{\lambda}\right)\right) \simeq \mathcal{R} G$. Portanto, $\mathcal{R} X=\bigoplus_{x_{\lambda} \in E}(\mathcal{R} G)_{x_{\lambda}}$.

Corolário 1.2.3. Se $H$ é um subgrupo de $G$ então $\mathcal{R} G$ é um $\mathcal{R} H$-módulo livre com base num conjunto $E$ de representantes para as $H$-órbitas em $G$ (que são as classes laterais à esquerda de $H$ em $G)$, isto é, $\mathcal{R} G=\bigoplus_{g \in E}(\mathcal{R} H)_{g}$. 
Demonstração: Temos que $G$ é um $H$-conjunto com a ação dada pela multiplicação dos elementos de $H$ por elementos de $G$ (isto é, $h . g:=h g$ ), esta ação é livre (pois $h . g=g \Leftrightarrow h=1$ ), e as $H$-órbitas em $G$ são $\{h . g ; h \in H\}=H . g$, para todo $g \in G$. Portanto, o resultado segue da proposição anterior.

Corolário 1.2.4. (a) Sejam X um G-conjunto livre e E um conjunto de representantes para as G-órbitas em $X$. Então $\mathcal{R} X$ é um $\mathcal{R} G$-módulo projetivo com base $E$.

(b) Se $H$ é um subgrupo de $G$ então $\mathcal{R} G$ é um $\mathcal{R} H$-módulo projetivo com base num conjunto $E$ de representantes para as $H$-órbitas em $G$.

\subsection{Resoluções Livres e Projetivas}

Definição 1.3.1. Seja $M$ um $R$-módulo arbitrário ( $R$ anel com unidade). Uma resolução de $M$ sobre $R$, ou uma $R$-resolução de $M$ é uma sequência exata de $R$-módulos

$$
F: \cdots \rightarrow F_{n+1} \stackrel{\partial_{n+1}}{\longrightarrow} F_{n} \stackrel{\partial_{n}}{\longrightarrow} F_{n-1} \stackrel{\partial_{n-1}}{\longrightarrow} \cdots
$$

a qual satisfaz as seguintes condições:

(i) $F_{-1}=M$

(ii) $F_{n}=0, \forall n<-1$.

Equivalentemente, uma resolução de $M$ sobre $R$ ou uma $R$-resolução de $M$ é uma sequência exata de $R$-módulos

$$
F: \cdots \rightarrow F_{2} \stackrel{\partial_{2}}{\longrightarrow} F_{1} \stackrel{\partial_{1}}{\longrightarrow} F_{0} \stackrel{\epsilon}{\longrightarrow} M \longrightarrow 0
$$

Definição 1.3.2. A aplicação $\epsilon: F_{0} \rightarrow M$ é chamada de aplicação aumentação. Se cada $F_{i}$ é livre, dizemos que a resolução é uma resolução livre. Se cada $C_{i}$ é projetivo, dizemos que a resolução é uma resolução projetiva.

Notação: $\epsilon: F \rightarrow M$ denotará uma resolução de $M$.

Observação 1.3.1. 1. Se considerarmos o complexos de cadeias

$$
F: \cdots \rightarrow F_{2} \stackrel{\partial_{2}}{\longrightarrow} F_{1} \stackrel{\partial_{1}}{\longrightarrow} F_{0} \longrightarrow 0 \longrightarrow 0 \longrightarrow \cdots
$$


podemos ver $\epsilon: F \rightarrow M$ como uma aplicação de cadeias, onde $M$ é identificado com o complexo de cadeias

$$
C: \cdots \rightarrow 0 \rightarrow C_{0}=M \rightarrow 0 \rightarrow \cdots
$$

2. Toda resolução livre é projetiva, pois todo módulo livre é projetivo ([11], Lema 7.2, p.22).

3. Se existir um inteiro $n$ tal que $F_{i}=0$, para $i>n$, dizemos que a resolução tem comprimento no máximo n. Neste caso, escrevemos simplesmente

$$
0 \rightarrow F_{n} \rightarrow \cdots \rightarrow F_{0} \rightarrow M \rightarrow 0
$$

4. Todo R-módulo $M$ possui uma resolução livre (projetiva) (ver [11], I, § 1 e 5, p.10 e 18).

Exemplo 1.3.1. Sejam $\mathcal{R}$ um anel comutativo com unidade e $G$ é cíclico infinito, $G=<t>\simeq \mathbb{Z}$. Considere $M=\mathcal{R}$ visto como $\mathcal{R} G$-módulo trivial. Então a sequência

$$
0 \rightarrow \mathcal{R} G \stackrel{\partial}{\rightarrow} \mathcal{R} G \stackrel{\epsilon}{\rightarrow} \mathcal{R} \rightarrow 0
$$

tal que d é a multiplicação por $(t-1)$ e e é a aplicação aumentação, ou seja,

$\partial\left(\sum_{i} r_{i} t^{i}\right)=(t-1)\left(\sum_{i} r_{i} t^{i}\right)=\sum_{i}\left(r_{i} t^{i+1}-r_{i} t^{i}\right)=\sum_{i}\left(r_{i-1}-r_{i}\right) t^{i}$

$\epsilon\left(\sum_{i} r_{i} t^{i}\right)=\sum_{i} r_{i}$

com $r_{i} \in R$ e $r_{i}=0$, exceto para um número finito de índices $i \in \mathbb{Z}$, é uma resolução livre de $M=\mathcal{R}$ sobre $R=\mathcal{R} G$.

De fato, sabemos que $\mathcal{R} G$ é $\mathcal{R} G$-módulo livre e temos que

(I) $\partial$ é monomorfismo, pois:

$\sum_{i} r_{i} t^{i} \in \operatorname{Ker}(\partial) \Rightarrow \partial\left(\sum_{i} r_{i} t^{i}\right)=0 \Rightarrow \sum_{i}\left(r_{i-1}-r_{i}\right) t^{i}=0 \Rightarrow r_{i-1}=r_{i} \Rightarrow r_{j}=$ $r_{i}, \forall i, j$.

Como $r_{i}=0$, exceto para um número finito de índices, então $r_{i}=0$, para todo i e dai, $\sum_{i} r_{i} t^{i}=0$. 
(II) $\operatorname{Im}(\partial)=\operatorname{Ker}(\epsilon)$ :

Temos que $(\epsilon \circ \partial)\left(\sum_{i} r_{i} t^{i}\right)=\epsilon\left(\sum_{i}\left(r_{i-1}-r_{i}\right) t^{i}\right)=\sum_{i} r_{i-1}-r_{i}=\sum_{i} r_{i-1}-$ $\sum_{i} r_{i}=0$ e daí, $\operatorname{Im}(\partial) \subset \operatorname{Ker}(\epsilon)$.

Agora, seja $y \in \operatorname{Ker}(\epsilon)$. Então $y=r_{0} t^{k}+r_{1} t^{k+1}+\cdots+r_{n} t^{k+n}, k \in \mathbb{Z}, n \in$ $\mathbb{N}, r_{0} \neq 0$ e $r_{n} \neq 0$ (aqui podemos ter $r_{i}=0$ se $\left.1 \leq i \leq n-1\right)$ e $\epsilon(y)=0$. Assim, $r_{0}+r_{1}+\cdots+r_{n}=0$, o que nos leva a $r_{n}=-r_{0}-r_{1}-\cdots-r_{n-1}$. Daí, existe $x=\left(-r_{0}\right) t^{k}+\left(-r_{0}-r_{1}\right) t^{k+1}+\cdots+\left(-r_{0}-r_{1}-\cdots-r_{n-1}\right) t^{k+(n-1)} \in \mathcal{R} G$ com $\partial x=(t-1) x=t x-x=\left(-r_{0}\right) t^{k+1}+\left(-r_{0}-r_{1}\right) t^{k+2}+\cdots+\left(-r_{0}-r_{1}-\right.$ $\left.\cdots-r_{n-1}\right) t^{k+n}+r_{0} t^{k}+\left(r_{0}+r_{1}\right) t^{k+1}+\cdots+\left(r_{0}+r_{1}+\cdots+r_{n-1}\right) t^{k+(n-1)}=$ $r_{0} t^{k}+r_{1} t^{k+1}+\cdots+r_{n} t^{k+n}=y . \operatorname{Logo}, \operatorname{Ker}(\epsilon) \subset \operatorname{Im}(\partial)$.

(III) $\epsilon$ é claramente um epimorfismo, pois $\epsilon$ é a aplicação aumentação.

$D e(\mathrm{I}),(\mathrm{II}) e(\mathrm{III})$

concluímos que a sequência dada inicialmente é exata.

Logo,

$$
0 \rightarrow \mathcal{R} G \stackrel{\partial}{\rightarrow} \mathcal{R} G \stackrel{\epsilon}{\rightarrow} \mathcal{R} \rightarrow 0
$$

é uma resolução livre de $\mathcal{R}$ sobre $\mathcal{R} G$.

Nosso objetivo agora é mostrar a unicidade de resoluções projetivas (para um módulo $M)$ a menos de equivalência de homotopia.

Lema 1.3.1. Consideremos o seguinte diagrama de homomorfismos de R-módulos

$$
\begin{gathered}
X \stackrel{d}{\rightarrow} Y \stackrel{c}{\rightarrow} Z \\
\exists h \downarrow \quad \downarrow_{j} \quad \downarrow_{k} \\
B \stackrel{f}{\rightarrow} C \stackrel{g}{\rightarrow} D
\end{gathered}
$$

onde X é projetivo, o quadrado YZCD é comutativo, a sequência de cima é semi-exata e a sequência de baixo é exata. Então existe um homomorfismo $h: X \rightarrow B$ satisfazendo $f \circ h=j \circ d$. 
Demonstração: Primeiramente, mostremos que $\operatorname{Im}(j \circ d) \subset \operatorname{Im}(f)$. Para isto, seja $x \in \operatorname{Im}(j \circ d)$. Deste modo, temos que existe $y \in X$ tal que $(j \circ d)(y)=x$. Como a sequência de cima é semi-exata, temos que $\operatorname{Im}(d) \subset \operatorname{Ker}(c)$. Assim, $d(y) \in \operatorname{Ker}(c)$ e daí, $c(d(y))=0$. Logo, $[k \circ c](d(y))=k[c(d(y))]=0$. Pela comutatividade do quadrado YZCD, temos que $k \circ c=g \circ j$. Logo, $(g \circ j)(d(y))=0$ e então $g[(j \circ d)(y)]=0$. Como $x=(j \circ d)(y)$, temos que $g(x)=0$ e portanto, $x \in \operatorname{Ker}(g)=\operatorname{Im}(f)$, pois a sequência de baixo é exata, o que implica que $x \in \operatorname{Im}(f)$.

Portanto, $\operatorname{Im}(j \circ d) \subset \operatorname{Im}(f)$.

Considerando então o diagrama abaixo e usando que X é projetivo existira $h: X \rightarrow B$ tal que $j \circ d=f \circ h$ e assim obtém-se o resultado.

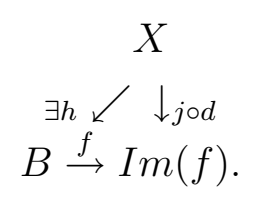

Proposição 1.3.1. Consideremos $X$ e $Y R$-módulos, $h: X \rightarrow Y$ um R-homomorfismo,

$$
\begin{aligned}
& C: \cdots \longrightarrow C_{n+1} \stackrel{\partial_{n+1}}{\longrightarrow} C_{n} \stackrel{\partial_{n}}{\longrightarrow} C_{n-1} \stackrel{\partial_{n-1}}{\longrightarrow} \cdots \longrightarrow C_{0} \longrightarrow X \longrightarrow 0 \quad e \\
& D: \cdots \longrightarrow D_{n+1} \stackrel{\partial^{\prime}{ }_{n+1}}{\longrightarrow} D_{n} \stackrel{\partial^{\prime}{ }_{n}}{\longrightarrow} D_{n-1} \stackrel{\partial^{\prime}{ }_{n-1}}{\longrightarrow} \cdots \longrightarrow D_{0} \longrightarrow Y \longrightarrow 0,
\end{aligned}
$$

resoluções projetivas de $X$ e $Y$, respectivamente.

Então existe um homomorfismo (aplicação de cadeias) $f=\left\{f_{n}: C_{n} \rightarrow D_{n} ; n \in \mathbb{Z}\right\}$, da sequência $C$ na sequência $D$ tal que $f_{-1}=h$.

Demonstração: Primeiro define-se $f_{-1}=h$. Como, para $n<-1, C_{n}=0$, tem-se que $f_{n}$ é unicamente definido, ou seja, $f_{n}=0$.

Para o caso $n=0$, considera-se o seguinte diagrama:

$$
\begin{array}{r}
C_{0} \stackrel{\partial_{0}}{\rightarrow} X \rightarrow 0 \\
\exists f_{0} \downarrow \quad \downarrow_{h=f_{-1}} \\
D_{0} \stackrel{\partial_{0}^{\prime}}{\rightarrow} Y \rightarrow 0
\end{array}
$$

Como $C_{0}$ é projetivo e $\partial_{0}^{\prime}: D_{0} \rightarrow Y$ é um epimorfismo, segue diretamente da definição de módulo projetivo que existe um homomorfismo $f_{0}: C_{0} \rightarrow D_{0}$ satisfazendo a seguinte relação de comutatividade $\partial_{0}^{\prime} \circ f_{0}=h \circ \partial_{0}=f_{-1} \circ \partial_{0}$.

Considere agora $n>0$ e suponha que $f_{m}: C_{m} \rightarrow D_{m}$ já tenha sido construído, para qualquer $m<n$, de tal maneira que o seguinte retângulo

$$
\begin{gathered}
C_{m} \stackrel{\partial_{m}}{\rightarrow} C_{m-1} \\
f_{m} \downarrow \\
D_{m} \stackrel{\downarrow^{\prime} m}{\rightarrow} D_{f_{m-1}}
\end{gathered}
$$


seja comutativo, para todo inteiro $m<n$. Considere o diagrama de homomorfismos:

$$
\begin{gathered}
C_{n} \stackrel{\partial_{n}}{\rightarrow} C_{n-1} \stackrel{\partial_{n-1}}{\longrightarrow} C_{n-2} \\
\exists f_{n} \downarrow \\
D_{n} \stackrel{{ }^{\prime}{ }_{f_{n-1}}}{\longrightarrow} D_{n-1} \stackrel{\partial_{n-1}^{\prime}}{\longrightarrow} D_{f_{n-2}} \\
\quad
\end{gathered}
$$

De acordo com o lema anterior, existe um homomorfismo $f_{n}: C_{n} \rightarrow D_{n}$ satisfazendo $\partial_{n}^{\prime} \circ f_{n}=f_{n-1} \circ \partial_{n}$. Isto completa a construção indutiva da aplicação de cadeia $f: C \rightarrow D$, o que conclui a demonstração.

Lema 1.3.2. Consideremos o seguinte diagrama de homomorfismos de $R$-módulos.

$$
\begin{gathered}
X \\
\exists k \swarrow \downarrow_{h} \\
B \stackrel{f}{\rightarrow} C \stackrel{g}{\rightarrow} D
\end{gathered}
$$

onde $X$ é projetivo, $g \circ h=0$ e a sequência $B \stackrel{f}{\rightarrow} C \stackrel{g}{\rightarrow} D$ é exata. Então existe um homomorfismo $k: X \rightarrow B$ satisfazendo $f \circ k=h$.

Demonstração: Tem-se por hipótese, que a sequência $B \stackrel{f}{\rightarrow} C \stackrel{g}{\rightarrow} D$ é exata. Logo, $\operatorname{Im}(f)=\operatorname{Ker}(g)$. Como $g \circ h=0$, temos que $\operatorname{Im}(h) \subset \operatorname{Ker}(g)=\operatorname{Im}(f)$.

Portanto, $\operatorname{Im}(h) \subset \operatorname{Im}(f)$, e podemos considerar o diagrama

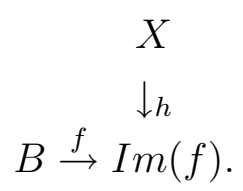

A existência do homomorfismo $k: X \rightarrow B$ é garantida pelo fato do módulo $\mathrm{X}$ ser projetivo. Além disso, $f \circ k=h$.

Proposição 1.3.2. Sejam $X, Y, h, C$ e D como na proposição anterior. Então quaisquer dois homomorfismos (aplicações de cadeias)

$$
\begin{aligned}
& f=\left\{f_{n}: C_{n} \rightarrow D_{n} ; n \in \mathbb{Z}\right\}, \\
& g=\left\{g_{n}: C_{n} \rightarrow D_{n} ; n \in \mathbb{Z}\right\},
\end{aligned}
$$

da sequência $C$ na sequência $D$, satisfazendo $f_{-1}=h=g_{-1}$, são homotópicos.

Demonstração: Devemos construir uma homotopia (homotopia de cadeias) $k: f \simeq$ $g: C \rightarrow D$. Desta forma, temos que construir, para todo n, um homomorfismo $k=\left\{k_{n}\right.$ : $\left.C_{n} \rightarrow D_{n+1}, \forall n \in \mathbb{Z}\right\}$ tal que a relação $\partial_{n+1}^{\prime} \circ k_{n}+k_{n-1} \circ \partial_{n}=f_{n}-g_{n}$ é verdadeira no seguinte diagrama: 


$$
\begin{aligned}
C_{n} \stackrel{\partial_{n}}{\longrightarrow} C_{n-1} \\
k_{n} \stackrel{\swarrow f_{n} \downarrow \downarrow_{g_{n}} \quad \swarrow k_{n-1}}{D_{n+1} \stackrel{\partial_{n+1}^{\prime}}{\longrightarrow} D_{n}}
\end{aligned}
$$

para qualquer $n \in \mathbb{Z}$.

Para todo $n<-1, k_{n}=0$ é o único homomorfismo de $C_{n}$ em $D_{n+1}$, já que $C_{n}=0$.

Para $n=-1$, temos $k_{-2}=0$ e $f_{n}-g_{n}=f_{-1}-g_{-1}=h-h=0$. Logo, neste caso, devemos construir um homomorfismo $k_{-1}: C_{-1}=X \rightarrow D_{0}$ satisfazendo $\partial_{0}^{\prime} \circ k_{-1}=0$. A existência de $k_{-1}$ é imediata, de fato, podemos tomar $k_{-1}=0$.

Agora, consideremos $n \geq 0$ e suponhamos já termos construído $k_{m}: C_{m} \rightarrow D_{m+1}$, para todo inteiro $m<n$, e que a relação $\partial_{m+1}^{\prime} \circ k_{m}+k_{m-1} \circ \partial_{m}=f_{m}-g_{m}$ é válida para todo inteiro $m<n$.

Consideremos o seguinte diagrama:

$$
\begin{aligned}
& C_{n} \quad \stackrel{\partial_{n}}{\longrightarrow} C_{n-1} \quad \stackrel{\partial_{n-1}}{\longrightarrow} C_{n-2}
\end{aligned}
$$

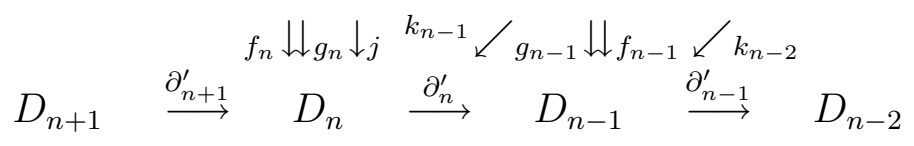

onde $j$ é o homomorfismo $j=f_{n}-g_{n}-k_{n-1} \circ \partial_{n}$.

Compondo $j$ com $\partial_{n}^{\prime}: D_{n} \rightarrow D_{n-1}$, obtemos $\partial_{n}^{\prime} \circ j: C_{n} \rightarrow D_{n-1}$ e $\partial_{n}^{\prime} \circ j=$ $\partial_{n}^{\prime} \circ f_{n}-\partial_{n}^{\prime} \circ g_{n}-\partial_{n}^{\prime} \circ k_{n-1} \circ \partial_{n}=f_{n-1} \circ \partial_{n}-g_{n-1} \circ \partial_{n}-\left(f_{n-1}-g_{n-1}-k_{n-2} \circ \partial_{n-1}\right) \circ \partial_{n}=$ $k_{n-2} \circ \partial_{n-1} \circ \partial_{n}=0$.

Como $C_{n}$ é projetivo e a sequência é exata, segue do lema anterior que existe um homomorfismo $k_{n}: C_{n} \rightarrow D_{n+1}$ satisfazendo a relação $\partial_{n+1}^{\prime} \circ k_{n}=j=f_{n}-g_{n}-k_{n-1} \circ \partial_{n}$. Isto implica que $\partial_{n+1}^{\prime} \circ k_{n}+k_{n-1} \circ \partial_{n}=f_{n}-g_{n}$, o que completa a construção indutiva da homotopia $k: f \simeq g: C \rightarrow D$.

Portanto, os homomorfismos (aplicações de cadeias) f e g são homotópicos.

Para o próximo resultado, vamos aplicar a Proposição 1.3.1 ao caso especial em que $X=Y=M$ e $h=i d_{M}$ é o homomorfismo idêntico. Neste caso, C e D são duas resoluções projetivas do mesmo módulo $\mathrm{M}$.

Teorema 1.3.1. Duas resoluções projetivas do mesmo módulo M são homotopicamente equivalentes.

Demonstração: Considere agora C e D duas resoluções projetivas do mesmo módulo M. Pela Proposição 1.3.1, existe um homomorfismo $f=\left\{f_{n}: C_{n} \rightarrow D_{n} ; n \in \mathbb{Z}\right\}$, da sequência $\mathrm{C}$ em $\mathrm{D}$, tal que $f_{-1}=h=i d_{M}$.

Similarmente, existe um homomorfismo $g=\left\{g_{n}: D_{n} \rightarrow C_{n} ; n \in \mathbb{Z}\right\}$ da sequência D em C, tal que $g_{-1}=h=i d_{M}$ e podemos construir o seguinte diagrama: 


$$
\begin{aligned}
& C: \cdots \longrightarrow C_{n+1} \stackrel{\partial_{n+1}}{\longrightarrow} C_{n} \stackrel{\partial_{n}}{\longrightarrow} C_{n-1} \stackrel{\partial_{n-1}}{\longrightarrow} \cdots \longrightarrow C_{0} \stackrel{\epsilon}{\longrightarrow} M \longrightarrow 0 \\
& f_{n+1} \downarrow \uparrow \uparrow_{n+1} \quad f_{n} \downarrow \uparrow g_{n} \quad f_{n-1} \downarrow \uparrow \uparrow_{g_{n-1}} \quad f_{0} \downarrow \uparrow_{g_{0}} \quad i d_{M} \downarrow \uparrow_{i d_{M}} \\
& D: \cdots \longrightarrow D_{n+1} \stackrel{\partial_{n+1}^{\prime}}{\longrightarrow} D_{n} \stackrel{\partial_{n}^{\prime}}{\longrightarrow} D_{n-1} \stackrel{\partial_{n-1}^{\prime}}{\longrightarrow} \cdots \longrightarrow D_{0} \stackrel{\epsilon^{\prime}}{\longrightarrow} M \longrightarrow 0
\end{aligned}
$$

Considere os homomorfismos de $\mathrm{C}$ em $\mathrm{C}$ dados pela composição $\tilde{g}=g \circ f=$ $\left\{g_{n} \circ f_{n}: C_{n} \rightarrow C_{n} ; n \in \mathbb{Z}\right\}$ e $i d_{C}=\left\{i d_{C_{n}}: C_{n} \rightarrow C_{n}, n \in \mathbb{Z}\right\}$. Como $\tilde{g}_{-1}=g_{-1} \circ f_{-1}=$ $i d_{M}=i d_{C_{-1}}$, segue da Proposição 1.3.2 (caso particular em que as resoluções são iguais) que $g \circ f \simeq i d_{C}$. Analogamente, considerando os homomorfismos de $\mathrm{D}$ em $\mathrm{D}, f \circ g$ e $i d_{D}$, concluímos que $f \circ g \simeq i d_{D}$. Assim, f e g são equivalências de homotopia. Portanto, $\mathrm{C}$ e D são homotopicamente equivalentes.

Observação 1.3.2. O teorema anterior nos diz que: "Duas resoluções projetivas de um mesmo módulo $M$ são 'iguais', a menos de uma equivalência de homotopia”. Note que a equivalência de homotopia $f: C \rightarrow D$, dada na demonstração do teorema, é uma aplicação que preserva aumentação, isto é, satisfaz $\epsilon^{\prime} \circ f_{0}=\epsilon$.

\subsection{Módulo de Homomorfismos}

Definição 1.4.1. Sejam $R$ um anel comutativo com unidade, $M$ e $N$ R-módulos arbitrários e considere o conjunto $\operatorname{Hom}_{R}(M, N):=\{f: M \rightarrow N ; f$ é homomorfismo $\}$. Definindo em $\operatorname{Hom}_{R}(M, N)$ uma adição, + , que a cada $f, g \in \operatorname{Hom}_{R}(M, N)$ associa o elemento $f+g$, com $(f+g)(m)=f(m)+g(m)$, para todo $m \in M$ e uma multiplicação,, tal que $: R \times \operatorname{Hom}_{R}(M, N) \rightarrow \operatorname{Hom}_{R}(M, N)$ e associa a cada elemento $(r, f) \in R \times \operatorname{Hom}_{R}(M, N)$ ao elemento $r f \in \operatorname{Hom}_{R}(M, N)$, com $(r f)(m)=r f(m)$ para todo $m \in M$, temos em $\operatorname{Hom}_{R}(M, N)$ uma estrutura de R-módulo, o qual é chamado módulo de homomorfismos do módulo $M$ no módulo $N$.

Observação 1.4.1. Quando $R$ não é comutativo, $r f$ não necessariamente pertence a $H_{\text {om }}(M, N)$ para $r \in R$. Nesse caso $H_{o m}(M, N)$ é visto apenas como um grupo abeliano, ou $\mathbb{Z}$-módulo, e resultados similares aos dados a $\operatorname{Hom}_{R}(M, N)$ quando $\operatorname{Hom}_{R}(M, N)$ for um $R$-módulo ( $R$ comutativo) são válidos para $\operatorname{Hom}_{R}(M, N)$ visto apenas como $\mathbb{Z}$ módulo.

Proposição 1.4.1. Para qualquer $R$-módulo $M$, temos $\operatorname{Hom}_{R}(R, M) \stackrel{\varrho}{\simeq} M$. 
Demonstração: Defina a aplicação

$$
\begin{aligned}
\varrho: \operatorname{Hom}_{R}(R, M) & \rightarrow M \\
f & \mapsto \varphi(f)=f(1) .
\end{aligned}
$$

Usando as operações definidas em $\operatorname{Hom}_{R}(R, M)$ verifica-se que $\varrho$ é um homomorfismo (de $R$-módulos ou simplesmente de $\mathbb{Z}$-módulo se $R$ é não comutativo). Verifiquemos que $\varrho$ é bijetor. De fato, tomando $m \in M$, do fato de $R$ ser um módulo livre gerado por $\{1\}$, segue que existe um único homomorfismo $f \in \operatorname{Hom}_{R}(R, M)$, tal que $\varrho(f)=f(1)=m$. Logo, $\varrho$ é um epimorfismo, da unicidade de $f$ segue que $\varrho$ é um monomorfismo e portanto $\varrho$ é isomorfismo.

Observação 1.4.2. Observemos que

$$
\begin{aligned}
\varphi: M & \rightarrow \operatorname{Hom}_{R}(R, M) \\
m & \mapsto \varphi_{m} ; \varphi_{m}(1)=m
\end{aligned}
$$

é o homomorfismo inverso de $\varrho$.

Definição 1.4.2. Sejam $f: M^{\prime} \rightarrow M$ e $g: N \rightarrow N^{\prime}$ homomorfismos de R-módulos e consideremos os módulos (ou grupos abelianos) $\operatorname{Hom}_{R}(M, N)$ e $\operatorname{Hom}_{R}\left(M^{\prime}, N^{\prime}\right)$.

Defina uma aplicação $v: \operatorname{Hom}_{R}(M, N) \rightarrow \operatorname{Hom}_{R}\left(M^{\prime}, N^{\prime}\right)$ tal que $v(h):=g \circ h \circ f$, para todo $h \in \operatorname{Hom}_{R}(M, N)$.

Essa aplicação é um homomorfismo de $\operatorname{Hom}_{R}(M, N)$ em $\operatorname{Hom}_{R}\left(M^{\prime}, N^{\prime}\right)$, o qual será denotado por Hom $(f, g)$.

Observação 1.4.3. 1. Se $f$ e g, forem os homomorfismos idênticos de $M$ e $N$, respectivamente, isto é, $f=i d_{M}: M \rightarrow M$ e $g=i d_{N}: N \rightarrow N$, então Hom $(f, g)=$ $i d_{H o m_{R}(M, N)}: \operatorname{Hom}_{R}(M, N) \rightarrow \operatorname{Hom}_{R}(M, N)$.

2. Se $f: M^{\prime} \rightarrow M, f^{\prime}: M^{\prime \prime} \rightarrow M^{\prime}, g: N \rightarrow N^{\prime}$ e $g^{\prime}: N^{\prime} \rightarrow N^{\prime \prime}$ são homomorfismos então Hom $\left(f \circ f^{\prime}, g^{\prime} \circ g\right)=\operatorname{Hom}\left(f^{\prime}, g^{\prime}\right) \circ \operatorname{Hom}(f, g)$.

3. Como uma consequência de 1. e 2. temos que se $f: M^{\prime} \rightarrow M$ e $g: N \rightarrow N^{\prime}$ são isomorfismos então Hom $(f, g)$ é um isomorfismo. 
Teorema 1.4.1. ([15], Teorema 8.4, p.70 ) Se $M=\sum_{\mu \in I} M_{\mu}$ e $N=\prod_{\nu \in J} N_{\nu}$ são R-módulos para todos $\mu \in I$ e $\nu \in J$, então $\operatorname{Hom}_{R}(M, N) \simeq \prod_{(\mu, \nu) \in I \times J} \operatorname{Hom}_{R}\left(M_{\mu}, N_{\nu}\right)$.

Observação 1.4.4. Em particular temos

1. Se $M=\bigoplus_{\mu \in I} M_{\mu}$ e $N$ são $R$-módulos para todo $\mu \in I$, então

$$
\operatorname{Hom}_{R}(M, N) \simeq \prod_{\mu \in I} \operatorname{Hom}_{R}\left(M_{\mu}, N\right)
$$

2. Se $N=\prod_{\nu \in I} M_{\mu}$ e $M$ são $R$-módulos para todo $\nu \in J$, então

$$
\operatorname{Hom}_{R}(M, N) \simeq \prod_{\nu \in I} \operatorname{Hom}_{R}\left(M, N_{\nu}\right)
$$

Teorema 1.4.2. ([15], Teorema 8.7, p.72, ou [22] Teorema 2.9, p.35) Seja M um Rmódulo arbitrário e

$$
X \stackrel{f}{\longrightarrow} Y \stackrel{g}{\longrightarrow} Z \longrightarrow 0
$$

uma sequência exata de $R$-módulos, então a sequência

$$
0 \longrightarrow \operatorname{Hom}_{R}(Z, M) \stackrel{g^{*}}{\longrightarrow} \operatorname{Hom}_{R}(Y, M) \stackrel{f^{*}}{\longrightarrow} \operatorname{Hom}_{R}(X, M),
$$

onde $f^{*}=\operatorname{Hom}(f, i)$ e $g^{*}=\operatorname{Hom}(g, i)$, com $i=i d_{M}$, é também exata.

Definição 1.4.3. Dizemos que uma sequência exata curta de $R$-módulos

$$
0 \longrightarrow X \stackrel{f}{\longrightarrow} Y \stackrel{g}{\longrightarrow} Z \longrightarrow 0
$$

cinde se existir uma aplicação $h: Z \rightarrow Y$ tal que $g \circ h=i d_{Z}$, ou equivalentemente $A=\operatorname{Im}(f)=\operatorname{Ker}(g)$ é um somando direto de $Y$ ([22], p.33).

Teorema 1.4.3. ([15], Teorema 8.8, p.74 ) Seja M um R-módulo arbitrário e

$$
0 \longrightarrow X \stackrel{f}{\longrightarrow} Y \stackrel{g}{\longrightarrow} Z \longrightarrow 0
$$

uma sequência exata curta de $R$-módulos que cinde, então a sequência

$$
0 \longrightarrow \operatorname{Hom}_{R}(Z, M) \stackrel{g^{*}}{\longrightarrow} \operatorname{Hom}_{R}(Y, M) \stackrel{f^{*}}{\longrightarrow} \operatorname{Hom}_{R}(X, M) \longrightarrow 0,
$$

onde $f^{*}=\operatorname{Hom}(f, i)$ e $g^{*}=\operatorname{Hom}(g, i)$, com $i=i d_{M}: M \rightarrow M$ o automorfismo idêntico de $M$, é também uma sequência exata curta que cinde.

Teorema 1.4.4. ([15], Teorema 8.9, p.74, ou [22], Teorema 2.9, p.35) Seja M um Rmódulo arbitrário e

$$
0 \longrightarrow X \stackrel{f}{\longrightarrow} Y \stackrel{g}{\longrightarrow} Z
$$


uma sequência exata de $R$-módulos, então a sequência

$$
0 \longrightarrow \operatorname{Hom}_{R}(M, X) \stackrel{g^{*}}{\longrightarrow} \operatorname{Hom}_{R}(M, Y) \stackrel{f^{*}}{\longrightarrow} \operatorname{Hom}_{R}(M, Z),
$$

onde $f_{*}=\operatorname{Hom}(i, f)$ e $g_{*}=\operatorname{Hom}(i, g)$, com $i=i d_{M}$, é também exata.

Teorema 1.4.5. ([15], Teorema 8.10, p.75) Seja $M$ um R-módulo arbitrário e

$$
0 \longrightarrow X \stackrel{f}{\longrightarrow} Y \stackrel{g}{\longrightarrow} Z \longrightarrow 0
$$

uma sequência exata curta de $R$-módulos que cinde, então a sequência

$$
0 \longrightarrow \operatorname{Hom}_{R}(M, X) \stackrel{f_{*}}{\longrightarrow} \operatorname{Hom}_{R}(M, Y) \stackrel{g_{*}}{\longrightarrow} \operatorname{Hom}_{R}(M, Z) \longrightarrow 0,
$$

onde $f_{*}=\operatorname{Hom}(i, f)$ e $g_{*}=\operatorname{Hom}(i, g)$, com $i=i d_{M}: M \rightarrow M$, é também uma sequência exata curta que cinde.

Teorema 1.4.6. ([15], Teorema 9.4, p.79) Para um $R$-módulo arbitrário X e seu endomorfismo identidade $i: X \rightarrow X$, as seguintes afirmações são equivalententes:

(a) X é projetivo.

(b) Toda sequência exata curta

$$
0 \longrightarrow U \stackrel{f}{\longrightarrow} V \stackrel{g}{\longrightarrow} X \longrightarrow 0
$$

de R-módulos cinde.

(c) X é isomorfo a um somando direto de um $R$-módulo livre.

(d) Para todo epimorfismo $g: A \rightarrow B$,

$$
g_{*}=\operatorname{Hom}(i, g): \operatorname{Hom}(X, A) \rightarrow \operatorname{Hom}(X, B)
$$

é também um epimorfismo.

(e) Para toda sequência exata curta

$$
0 \longrightarrow A \stackrel{f}{\longrightarrow} B \stackrel{g}{\longrightarrow} C \longrightarrow 0,
$$

de R-módulos, a sequência

$$
0 \longrightarrow \operatorname{Hom}_{R}(X, A) \stackrel{f_{*}}{\longrightarrow} \operatorname{Hom}_{R}(X, B) \stackrel{g_{*}}{\longrightarrow} \operatorname{Hom}_{R}(X, C) \longrightarrow 0,
$$

$\operatorname{com} f_{*}=\operatorname{Hom}(i, f)$ e $g_{*}=\operatorname{Hom}(i, g)$ é também uma sequência exata curta. 


\subsection{Produto Tensorial}

Definição 1.5.1. Sejam $R$ um anel, $M$ um $R$-módulo à direita, $N$ um $R$-módulo à esquerda e $G$ um grupo abeliano. Uma aplicação biaditiva do produto cartesiano $M \times N$ em $G$ é uma aplicação $\varphi: M \times N \longrightarrow G$, tal que para quaisquer, $m, m^{\prime} \in M, n, n^{\prime} \in N$ e $r \in R$ satisfaz

1. $\varphi\left(m+m^{\prime}, n\right)=\varphi(m, n)+\varphi\left(m^{\prime}, n\right)$.

2. $\varphi\left(m, n+n^{\prime}\right)=\varphi(m, n)+\varphi\left(m, n^{\prime}\right)$.

3. $\varphi(m r, n)=\varphi(m, r n)$.

Definição 1.5.2. Sejam $M, N$ e $R$ como na definição anterior. O produto tensorial de $M$ e $N$ sobre $R$, o qual é denotado por $M \otimes_{R} N$, é um grupo abeliano junto com uma aplicação biaditiva, $\otimes: M \times N \longrightarrow M \otimes_{R} N$, que possui a seguinte propriedade universal: Para qualquer grupo abeliano $G$ e toda aplicação bilinear, $f: M \times N \longrightarrow G$, existe um único homomorfismo de grupos, $\tilde{f}: M \otimes_{R} N \longrightarrow G$, tal que $\tilde{f} \circ \otimes=f$.

Para cada $m \in M$ e $n \in N$, o elemento $\otimes(m, n)$ será denotado por $m \otimes n$, e devido a linearidade de $\otimes$ temos, para $m_{1}, m_{2} \in M, n_{1}, n_{2} \in N$ e $r \in R$ :

1. $\left(m_{1}+m_{2}\right) \otimes n=m_{1} \otimes n+m_{2} \otimes n$.

2. $m \otimes\left(n_{1}+n_{2}\right)=m \otimes n_{1}+m \otimes n_{2}$.

3. $m r \otimes n=m \otimes r n$.

Em particular, são válidas as seguintes propriedades:

1. $0 \otimes n=0=m \otimes 0$.

2. $(-m) \otimes n=-(m \otimes n)=m \otimes(-n)$.

Para todo $m \in M$ e $n \in N$.

Observação 1.5.1. 1. Seja $f: R \rightarrow S$ um homomorfismo de anéis e $M$ um $R$-módulo à esquerda. Então identificando $s r:=s f(r)$ temos que $S$ é um $R$-módulo à direita. Portanto podemos produzir o produto tensorial $S \otimes_{R} M$, o qual é um $S$-módulo, quando identificamos $a(b \otimes m):=(a b) \otimes m$, para $a, b \in S$.

2. Se $R$ é um anel comutativo e $M, N R$-módulos arbitrários então $M \otimes_{R} N$ é um R-módulo. 
3. A estrutura de módulos sobre $S \otimes_{R} M$ e $M \otimes_{R} N$ em 1. e 2. respectivamente, são casos especiais do seguinte: Sejam $M$ um $S-R$-bimódulo, ou seja, um módulo que tem estrutura de $S$-módulo e R-módulo, com $(s m) r=s(m r)$. Então $M \otimes_{R} N$ é um $S$-módulo identificando $s(m \otimes n):=(s m) \otimes n$. 


\section{CAPÍTULO 2}

\section{GRUPOS DE (CO)HOMOLOGIA}

\section{ABSOLUTA E RELATIVA}

Neste capítulo veremos os conceitos de (co)homologia absoluta e relativa e alguns outros conceitos e resultados importantes para o desenvolvimento desse trabalho, dentre eles a aplicação restrição, o Lema de Shapiro, par grupo $(G, \mathcal{S})$ e sequência exata longa em cohomologia para um par grupo. A referência principal para o estudo de (co)homologia absoluta de grupos é [11]. Para (co)homologia relativa a referência é [10]. Embora a (co)homologia de grupos possa ser definida considerando $\mathcal{R} G$-módulos, com $\mathcal{R}$ um anel qualquer comutativo com unidade, trabalharemos apenas com o anel $\mathcal{R} G$ para $\mathcal{R}=\mathbb{Z}$ (o anel dos inteiros), ou $\mathcal{R}=\mathbb{Z}_{2}$ (o corpo com dois elementos), que denotaremos simplesmente por $R G$. Assim, sempre que aparecer $R G, R$ estará indicando $\mathbb{Z}$ ou $\mathbb{Z}_{2}$ (observe no entanto, que $R$ também foi usado no capítulo anterior para indicar um anel qualquer com unidade).

\subsection{Grupos de (Co)homologia Absoluta}

Sejam $G$ um grupo, $M$ um $R G$-módulo à esquerda e

$$
\cdots \stackrel{\partial_{n-1}}{\longrightarrow} F_{n} \stackrel{\partial n}{\longrightarrow} \cdots \stackrel{\partial_{2}}{\longrightarrow} F_{1} \stackrel{\partial_{1}}{\longrightarrow} F_{0} \stackrel{\epsilon}{\longrightarrow} R \longrightarrow 0
$$

uma resolução projetiva de $R$ sobre $R G$, que denotaremos simplesmente por $\epsilon: F \rightarrow R$.

Definição 2.1.1. O n-ésimo grupo de homologia de $G$ com coeficientes em $M, H_{n}(G ; M)$, é definido por $H_{n}\left(F \otimes_{R G} M\right)$, onde $F \otimes_{R G} M$ é a sequência (complexo de cadeias)

$$
\cdots \stackrel{\bar{\partial}_{n+1}}{\longrightarrow} F_{n} \otimes_{R G} M \stackrel{\bar{\partial}_{n}}{\longrightarrow} F_{n-1} \otimes_{R G} M \stackrel{\bar{\partial}_{n-1}}{\longrightarrow} \cdots \stackrel{\bar{\partial}_{1}}{\longrightarrow} F_{0} \otimes_{R G} M \longrightarrow 0
$$

$\operatorname{com} \bar{\partial}_{n}=\partial_{n} \otimes i d_{M}, n \geq 1$ e $\bar{\partial}_{0}=0$. 
O n-ésimo grupo de cohomologia de $G$ com coeficientes em $M, H^{n}(G ; M)$, é definido por $H^{n}\left(\operatorname{Hom}_{R G}(F, M)\right)$, onde $\operatorname{Hom}_{R G}(F, M)$ é a sequência (complexo de cocadeias) $0 \longrightarrow \operatorname{Hom}_{R G}\left(F_{0}, M\right) \stackrel{\delta^{0}}{\longrightarrow} \operatorname{Hom}_{R G}\left(F_{1}, M\right) \stackrel{\delta^{1}}{\longrightarrow} \cdots \stackrel{\delta^{n-1}}{\longrightarrow} \operatorname{Hom}_{R G}\left(F_{n}, M\right) \stackrel{\delta^{n}}{\longrightarrow} \cdots$ com $\delta^{n}(f)=f \circ \partial_{n+1}$ para todo $f \in \operatorname{Hom}_{R G}\left(F_{n}, M\right)$.

Observação 2.1.1. Sabemos que todo $\mathbb{Z}_{2} G$-módulo é um $\mathbb{Z} G$-módulo, e pode-se mostrar que se $M$ é um $\mathbb{Z}_{2} G$-módulo então os grupos de (co)homologia de $G$ (sobre $\mathbb{Z}_{2}$ ) com $M$ visto como um $\mathbb{Z}_{2} G$-módulo e os grupos de (co)homologia de $G$ (sobre $\mathbb{Z}$ ) com $M$ visto como $\mathbb{Z} G$-módulo são isomorfos. Isto segue do fato que, se $\epsilon: F \rightarrow \mathbb{Z}$ é uma resolução projetiva de $\mathbb{Z}$ sobre $\mathbb{Z} G$ então $\epsilon^{\prime}=\epsilon \otimes i d: F^{\prime}=F \otimes_{\mathbb{Z}} \mathbb{Z}_{2} \rightarrow \mathbb{Z} \otimes_{\mathbb{Z}} \mathbb{Z}_{2} \simeq \mathbb{Z}_{2}$ é uma resolução projetiva de $\mathbb{Z}_{2}$ sobre $\mathbb{Z}_{2} G, \gamma: \operatorname{Hom}_{\mathbb{Z} G}(F, M) \rightarrow \operatorname{Hom}_{\mathbb{Z}_{2} G}\left(F^{\prime}, M\right) ; f \mapsto \gamma(f)$ tal que $\gamma(f)(x \otimes 1):=f(x)$, e $\chi: F \otimes_{\mathbb{Z}_{2} G} M \rightarrow F^{\prime} \otimes_{\mathbb{Z}_{2} G} M ; x \otimes m \mapsto(x \otimes 1) \otimes m$, são isomorfismos.

Observação 2.1.2. Como $T_{1}=\operatorname{Hom}_{R G}(-, M)$ e $T_{2}=\left(-\otimes_{R G} M\right)$ são funtores aditivos (contravariante e covariante, respectivamente), eles preservam aplicação de cadeias e segue do Teorema 1.3.1 e da Proposição 1.1.3 que os grupos de cohomologia e homologia independem da resolução projetiva escolhida. Mais precisamente, se $\epsilon: F \rightarrow R$ e $\epsilon^{\prime}: F^{\prime} \rightarrow R$ são resoluções projetivas de $R$ sobre $R G$, então os complexos de (co)cadeias $T_{i}(F)$ e $T_{i}\left(F^{\prime}\right), i \in\{1,2\}$ são homotopicamente equivalentes e assim, $H^{n}\left(T_{1}(F)\right) \simeq H^{n}\left(T_{1}\left(F^{\prime}\right)\right)$ e $H_{n}\left(T_{2}(F)\right) \simeq H_{n}\left(T_{2}\left(F^{\prime}\right)\right)$, donde segue o resultado.

Observação 2.1.3. $H^{n}(G ; M)=0$ e $H_{n}(G ; M)=0$, se $n<0$.

Definição 2.1.2. Sejam G um grupo e $M$ um $R G$-módulo (à esquerda). O grupo de invariantes de $M$, denotado por $M^{G}$, é definido por:

$$
M^{G}=\{m \in M ; g \cdot m=m, \forall g \in G\}
$$

Observação 2.1.4. 1. Se a ação de $G$ em $M$ é trivial, isto é, g.m $=m$, para qualquer $m \in M$, então $M^{G}=M$.

2. É claro que para todo $R G$-módulo $M$, a G-ação sobre $M$ induz a G-ação trivial sobre $M^{G}$ e assim, $M^{G}$ é um $R G$-módulo trivial, e ainda $M^{G}$ é o maior submódulo de $M$ no qual $G$ atua trivialmente.

Definição 2.1.3. O grupo de coinvariantes de $M$, denotado por $M_{G}$, é definido por: 


$$
M_{G}=\frac{M}{\langle g \cdot m-m ; g \in G \text { e } m \in M\rangle},
$$

onde $\langle g . m-m ; g \in G$ e $m \in M\rangle$ é o submódulo de $M$ gerado pelos elementos $g . m-m$, com $g \in G$ e $m \in M$.

Observação 2.1.5. Se a ação de $G$ em $M$ é trivial, então $M_{G}=\frac{M}{\{0\}}=M$.

Observação 2.1.6. Claramente se $M$ é um RG-módulo à esquerda então podemos ver $M$ como um RG-módulo à direita por considerar $m . g:=g^{-1} . m$.

Definição 2.1.4. (Ação Diagonal) Sejam $M$ e $N$ RG-módulos (á esquerda). Considerando $M$ e $N$ como $R$-módulos podemos definir sobre $\operatorname{Hom}_{R}(M, N)$ e sobre $M \otimes_{R} N$ $G$-ações (á esquerda), denominadas ações diagonais, de modo a torná-los RG-módulos (á esquerda). Essas ações, as quais são induzidas da ação de $G$ sobre $M$ e $N$, são definidas da seguinte forma:

$$
\begin{aligned}
G \times \operatorname{Hom}_{R}(M, N) & \rightarrow \operatorname{Hom}_{R}(M, N) \\
(g, f) & \mapsto g \cdot f ;(g \cdot f)(m)=g \cdot f\left(g^{-1} \cdot m\right), \forall m \in M, \\
G \times\left(M \otimes_{R} N\right) & \rightarrow M \otimes_{R} N \\
(g, m \otimes n) & \mapsto g \cdot(m \otimes n):=g \cdot m \otimes g \cdot n .
\end{aligned}
$$

Proposição 2.1.1. $\left(\operatorname{Hom}_{R}(M, N)\right)^{G}=\operatorname{Hom}_{R G}(M, N)$.

Demonstração: Sejam $g \in G$ e $f \in \operatorname{Hom}_{R}(M, N)$. Temos que

$$
\begin{aligned}
g . f=f & \Leftrightarrow(g \cdot f)(m)=f(m), \forall m \in M \\
& \Leftrightarrow g \cdot f\left(g^{-1} \cdot m\right)=f(m), \forall m \in M \\
& \Leftrightarrow g \cdot f\left(m^{\prime}\right)=f\left(g \cdot m^{\prime}\right), \forall m^{\prime} \in M\left(m^{\prime}=g^{-1} \cdot m\right) .
\end{aligned}
$$

$\left.\operatorname{Logo}_{(H o m}(M, N)\right)^{G}=\left\{f \in H_{o m}(M, N) ; g \cdot f(m)=f(g \cdot m)\right\}=H_{R o m}(M, N)$.

Corolário 2.1.1. Se $R$ é visto como $R G$-módulo trivial (à esquerda) então $\operatorname{Hom}_{R G}(R, M)=\left(\operatorname{Hom}_{R}(R, M)\right)^{G} \simeq M^{G}$ como grupos (e como RG-módulos triviais).

Proposição 2.1.2. Se $M$ é um $R G$-módulo então $H^{0}(G ; M) \simeq M^{G}$ e $H_{0}(G ; M) \simeq M_{G}$. 


\section{Demonstração: Seja}

$$
\cdots \stackrel{\partial_{n-1}}{\longrightarrow} F_{n} \stackrel{\partial n}{\longrightarrow} \cdots \stackrel{\partial_{2}}{\longrightarrow} F_{1} \stackrel{\partial_{1}}{\longrightarrow} F_{0} \stackrel{\epsilon}{\longrightarrow} R \longrightarrow 0
$$

uma resolução projetiva de $R$ sobre $R G$.

Pelo Teorema 1.4.2 o funtor $\operatorname{Hom}_{R G}(-, M)$ é exato à esquerda, e deste modo segue que a sequência

$$
\begin{gathered}
0 \longrightarrow \operatorname{Hom}_{R G}(R, M) \stackrel{\epsilon^{*}}{\longrightarrow} \operatorname{Hom}_{R G}\left(F_{0}, M\right) \stackrel{\delta^{0}}{\longrightarrow} \operatorname{Hom}_{R G}\left(F_{1}, M\right) \stackrel{\delta^{1}}{\longrightarrow} \cdots \\
0
\end{gathered}
$$

é exata à esquerda, $\log 0 \epsilon^{*}$ é monomorfismo e $\operatorname{Im}\left(\epsilon^{*}\right)=\operatorname{Ker}\left(\delta^{0}\right)$.

Usando que $M \stackrel{\varphi}{\simeq} \operatorname{Hom}_{R}(R, M)$ (ver Proposição 1.4.1) e que $\epsilon^{*}$ é injetora, obtemos $M^{G} \simeq\left(\operatorname{Hom}_{R}(R, M)\right)^{G}=\operatorname{Hom}_{R G}(R, M) \stackrel{\epsilon^{*}}{\simeq} \operatorname{Im}\left(\epsilon^{*}\right)=\operatorname{Ker}\left(\delta^{0}\right)=\frac{\operatorname{Ker}\left(\delta^{0}\right)}{\operatorname{Im}\left(\delta^{-1}\right)}=H^{0}(G ; M)$.

O isomorfismo $M^{G} \stackrel{\alpha}{\simeq} H^{0}(G ; M)$ é dado explicitamente por

$$
\begin{aligned}
\alpha: M^{G} & \rightarrow H^{0}(G ; M) \\
m & \mapsto \epsilon\left(\varphi_{m}\right)=\varphi_{m} \circ \epsilon
\end{aligned}
$$

onde $\varphi_{m}: R \rightarrow M$ é tal que $\varphi_{m}(1)=m$, sendo 1 a unidade do anel $R$.

Seu inverso $\beta$ é dado por

$$
\begin{aligned}
\beta: H^{0}(G ; M)=\operatorname{Im}\left(\epsilon^{*}\right) & \rightarrow M^{G} \\
\epsilon^{*}(f) & \mapsto f(1) .
\end{aligned}
$$

O resultado para homologia é obtido de forma similar usando o fato de o funtor - $\otimes_{R G} M$ ser exato à direita (ver [11], III, Proposição 6.1, p.71).

Corolário 2.1.2. Se $M$ é um $R G$-módulo trivial então $H^{0}(G ; M) \simeq M$ e $H_{0}(G ; M) \simeq M$.

Exemplo 2.1.1. Apresentamos a seguir os grupos de (co)homologia de $G=<t>\simeq \mathbb{Z}$ para alguns módulos particulares.

(i) Se $M$ é um RG-módulo então $H^{0}(G ; M)=M^{G}=H_{1}(G ; M), H_{0}(G ; M)=M_{G}=$ $H^{1}(G ; M)$ e $H^{i}(G ; M)=0=H_{i}(G ; M)$, para $i \geq 2$. Em particular, se $M$ é um 
$R G$-módulo trivial, $H^{0}(G ; M)=H^{1}(G ; M)=H_{0}(G ; M)=H_{1}(G ; M)=M$. Isto é facilmente obtido usando a definição de (co)homologia e a resolução projetiva

$$
0 \rightarrow R G \stackrel{\partial=t-1}{\longrightarrow} R G \stackrel{\epsilon}{\rightarrow} R \rightarrow 0
$$

de $G \simeq \mathbb{Z}$ sobre $R G$ (vide Exemplo 1.3.1).

(ii) Seja $M=\mathbb{Z} G$ visto como $\mathbb{Z} G$-módulo com a $G$-ação natural: $t^{k} \cdot\left(r t^{k^{\prime}}\right):=r t^{k+k^{\prime}}$, para todos $t^{k}, t^{k^{\prime}} \in G$ e $r \in R$.

Então $H^{0}(G ; \mathbb{Z} G) \simeq(\mathbb{Z} G)^{G} \simeq 0\left(\right.$ pois $t^{k} \cdot t^{k^{\prime}} \neq t^{k}$ se $\left.t^{k} \neq 1\right)$.

Sabemos por $(i)$ que $H^{1}(G ; \mathbb{Z} G) \simeq(\mathbb{Z} G)_{G}$. Denotemos por $I=\left\langle t^{k} . z-z ; t^{k} \in\right.$ $G, z \in \mathbb{Z} G\rangle$ e $\bar{x}=x+I \in \frac{\mathbb{Z} G}{I}=(\mathbb{Z} G)_{G}$.

Consideremos $y=t^{k} \in \mathbb{Z} G$ com $k>0$. Como $t^{k}-1=(t-1)\left(t^{k-1}+\cdots+1\right) \in I$, segue que $\overline{t^{k}-1}=\overline{0}$, e assim $\bar{y}=\overline{t^{k}}=\overline{1} \mathrm{em}(\mathbb{Z} G)_{G}$.

Agora, se $y=t^{-k} \in \mathbb{Z} G$, com $k>0$, também $\overline{t^{-k}}=\overline{1}$, pois $t^{-k}=\left(1-t^{k}\right) t^{-k}+1 \in I$ $e \overline{1-t^{k}}=\overline{0}$.

Assim, para todo $x \in \mathbb{Z} G, x=r_{0} t^{k}+r_{1} t^{k+1}+\cdots+r_{n} t^{k+n}$, com $k \in \mathbb{Z}, n \geq 0 e$ $r_{i} \in \mathbb{Z}$, temos $\bar{x}=\overline{\left(r_{0}+r_{1}+\cdots+r_{n}\right)}=r . \overline{1}$, onde $r=r_{0}+r_{1}+\cdots+r_{n} \in \mathbb{Z}$. Daí, $H^{1}(G ; \mathbb{Z} G) \simeq \mathbb{Z}$

Logo, $H^{i}(G ; \mathbb{Z} G) \simeq\left\{\begin{array}{cc}0, & i=0 \\ \mathbb{Z}, & i=1 \\ 0, & i \geq 2\end{array}\right.$.

\subsubsection{Restrição e (Co)extensão de Escalares}

Consideremos $A, B$ anéis e $k: A \rightarrow B$ um homomorfismo de anéis.

Se $M$ é um $B$-módulo (à esquerda) sempre podemos ver $M$ como um $A$-módulo (à esquerda) definindo em $M$ a $A$-multiplicação:

$$
\begin{aligned}
A \times M & \rightarrow M \\
(a, m) & \mapsto a \cdot m:=k(a) . m
\end{aligned}
$$

Neste caso, $M$ é dito ser um $A$-módulo por restrição de escalares (via $k$ ) e é denotado por $\operatorname{Res}_{A}^{B} M$.

Considere agora $M$ um $A$-módulo (à esquerda), através de um homomorfismo $k: A \rightarrow B$. Iremos obter, a partir de $M$, os $B$-módulos (à esquerda) $B \otimes_{A} M$ e $\operatorname{Hom}_{A}(B, M)$. 
1. No primeiro caso, podemos ver $B$ como um $A$-módulo (à direita), por restrição de escalares, através da ação:

$$
\begin{aligned}
\tau: B \times A & \rightarrow B \\
(b, a) & \mapsto b \cdot a=b k(a)
\end{aligned}
$$

onde $b k(a)$ é a multiplicação do anel.

Assim podemos considerar o produto tensorial $B \otimes_{A} M$.

Nosso objetivo é definir em $B \otimes_{A} M$ uma $B$-ação (à esquerda) para que este se torne um $B$-módulo.

Definimos, para isso

$$
\begin{aligned}
\psi: B \times\left(B \otimes_{A} M\right) & \rightarrow B \otimes_{A} M \\
\left(b, b^{\prime} \otimes m\right) & \mapsto b .\left(b^{\prime} \otimes m\right):=b b^{\prime} \otimes m .
\end{aligned}
$$

Pode-se provar que $\psi$ está bem definida e é uma $B$-ação.

O $B$-módulo (à esquerda) $B \otimes_{A} M$ é dito ser obtido de $M$ por extensão de escalares de $A$ para $B$ (via $k$ ).

2. Agora, se $M$ é um $A$-módulo (à esquerda), podemos obter um $B$-módulo (à esquerda), $\operatorname{Hom}_{A}(B, M)$, bastante relacionado $\operatorname{com} M$, da seguinte maneira:

Por restrição, podemos ver $B$ como um $A$-módulo (à esquerda)

$$
\begin{aligned}
A \times B & \rightarrow B \\
(a, b) & \mapsto a . b:=k(a) . b
\end{aligned}
$$

e assim, faz sentido considerar $\operatorname{Hom}_{A}(B, M)$.

Pode-se mostrar que a aplicação

$$
\begin{aligned}
A \times \operatorname{Hom}_{A}(B, M) & \rightarrow \operatorname{Hom}_{A}(B, M) \\
(b, f) & \mapsto b f ;(b f)\left(b^{\prime}\right):=f\left(b^{\prime} b\right)
\end{aligned}
$$

está bem definida e é uma $B$-multiplicação.

O $B$-módulo $\operatorname{Hom}_{A}(B, M)$ é dito ser obtido de $M$ por coextensão de escalares de $A$ para $B($ via $k)$.

Proposição 2.1.3. ([11], III, §3, p.64) Sejam $k: A \rightarrow B$ um homomorfismo de anéis, 
$N$ um B-módulo (à esquerda) e $M$ um A-módulo (à esquerda) então $\operatorname{Hom}_{A}(N, M) \simeq$ $\operatorname{Hom}_{B}\left(N, H_{A}(B, M)\right)$ e $\operatorname{Hom}_{A}(M, N) \simeq \operatorname{Hom}_{B}\left(B \otimes_{A} M, N\right)$, como grupos.

\subsubsection{Módulos Induzidos e Módulos Coinduzidos}

Considere agora um caso particular da construção anterior, tomando $A=R H$ e $B=R G$, com $G$ um grupo, $H$ um subgrupo de $G$, e o homomorfismo de anéis $\tilde{\alpha}: R H \rightarrow R G$ induzido da inclusão $\alpha: H \rightarrow G$.

Se $M$ é um $R G$-módulo, podemos ver $M$ como um $R H$-módulo por restrição de escalares (via $\tilde{\alpha}$ ). O $R H$-módulo $M$ assim obtido é usualmente denotado por $\operatorname{Res}_{H}^{G} M$, ou simplesmente, por $M$ caso não haja confusão.

Seja $M$ um $R H$-módulo. O $R G$-módulo obtido de $M$ por extensão de escalares, $R G \otimes_{R H} M$, será denotado por $I n d_{H}^{G} M$, e neste caso, a extensão de escalares é chamada indução, e a ação é dada por:

$$
\begin{aligned}
G \times \operatorname{Ind}_{H}^{G} M & \rightarrow \operatorname{Ind}_{H}^{G} M \\
\left(g, g^{\prime} \otimes m\right) & \mapsto\left(g g^{\prime}\right) \otimes m .
\end{aligned}
$$

Da mesma forma, considerando $M$ um $R H$-módulo, o $R G$-módulo obtido de $M$ por coextensão de escalares, $\operatorname{Hom}_{R H}(R G, M)$, será denotado por $\operatorname{Coind}_{H}^{G} M$.

A coextensão de escalares, nesse caso, é chamada coindução.

Aqui a $G$-ação é dada por:

$$
\begin{aligned}
G \times \operatorname{Coind}_{H}^{G} M & \rightarrow \operatorname{Coind}_{H}^{G} M \\
(g, f) & \mapsto g \cdot f ;(g \cdot f)\left(g^{\prime}\right)=f\left(g^{\prime} \cdot g\right)
\end{aligned}
$$

Observação 2.1.7. Se $H=\{1\}$, temos que $R H \simeq R$. Deste modo $\operatorname{Ind}_{\{1\}}^{G} M=R G \otimes_{R} M$ e $\operatorname{Coind}_{\{1\}}^{G} M=\operatorname{Hom}_{R}(R G, M)$. Estes RG-módulos são chamados módulo induzido e módulo coinduzido, respectivamente.

Proposição 2.1.4. ([11], p. 70) Sejam $G$ um grupo, $H$ um subgrupo de $G, M$ um RHmódulo e E um conjunto de representantes para as classes laterais à esquerda de $H$ em G. Então:

1. O RG-módulo Ind ${ }_{H}^{G} M$ contém $M$ como $R H$-submódulo e é a soma direta de transformadas $g M$, onde g varia em $E$, ou seja,

$$
\operatorname{Ind}_{H}^{G} M=\bigoplus_{g \in E} g M
$$


2. O RG-módulo Coind ${ }_{H}^{G} M$ admite uma decomposição em produto direto, ou seja, existe uma família de sobrejeções $\pi_{g}: \operatorname{Coind}_{H}^{G} M \rightarrow g M, g \in E$, tal que a aplicação correspondente $u:$ Coind $_{H}^{G} M \rightarrow \prod_{g \in E} g M$ é um isomorfismo.

Proposição 2.1.5. Seja $M$ um RG-módulo. Então temos os seguintes isomorfismos de $R G$-módulos:

1. $\operatorname{Ind}_{H}^{G} M \simeq R(G / H) \otimes_{R} M$.

2. $\operatorname{Coind}_{H}^{G} M \simeq \operatorname{Hom}_{R}(R(G / H), M)$,

onde ação de G no produto tensorial e no Hom é a diagonal, e no primeiro membro $M$ é visto como um RH-módulo por restrição de escalares.

\section{Demonstração:}

1. Definimos os seguintes homomorfismos de grupos:

$$
\begin{aligned}
\varphi: R G \otimes_{R H} M & \rightarrow R(G / H) \otimes_{R} M \\
g \otimes m & \mapsto \bar{g} \otimes g m \\
\psi: R(G / H) \otimes_{R} M & \rightarrow R G \otimes_{R H} M \\
\bar{g} \otimes m & \mapsto g \otimes g^{-1} m,
\end{aligned}
$$

onde $\bar{g}$ indica a classe gH.

A partir disso, mostra-se que $\varphi$ é o inverso de $\psi$, e além disso, que $g \cdot \psi(\bar{g} \otimes m)=$ $\psi(g \cdot(\bar{g} \otimes m))$.

2. Nesse caso, são definidos os homomorfismos:

$$
\begin{aligned}
\phi: \operatorname{Hom}_{R H}(R G, M) & \rightarrow \operatorname{Hom}_{R}(R(G / H), M) \\
f & \mapsto \phi(f) ; \phi(f)(g H):=g f\left(g^{-1}\right), \\
\gamma: \operatorname{Hom}_{R}(R(G / H), M) & \rightarrow H_{R H}(R G, M) \\
f & \mapsto \gamma(f) ; \gamma(f)(g)=g f\left(g^{-1} H\right) .
\end{aligned}
$$

Mostra-se que $\phi$ é um homomorfismo de $R G$-módulos e $\gamma$ seu inverso.

Corolário 2.1.3. $\operatorname{Ind}_{H}^{G} R \simeq R(G / H)$ como $R G$-módulos. 
Proposição 2.1.6. ([11], III.5, Proposição 5.9, p.70) Se $[G: H]<\infty$ então $\operatorname{Ind}_{H}^{G} M \simeq$ $\operatorname{Coind}_{H}^{G} M$, como RG-módulos.

Proposição 2.1.7. ([11], Exercício 4, p.71) Se $[G: H]=\infty$ então $\left(\operatorname{Ind}_{H}^{G}\right)^{G}=0$, para qualquer $R H$-módulo $M$.

\section{$2.2 \quad H^{*}$ como funtor de duas variáveis}

Os tópicos desenvolvidos aqui são válidos também para $\mathbb{Z} G$-módulos. De fato, poderíamos considerar $R G$-módulos, onde $R$ é um anel comutativo com unidade. No entanto, nos restringiremos apenas ao caso de maior interesse, a saber, $\mathbb{Z}_{2} G$-módulos.

Vamos considerar pares $(G, M)$ e $\left(G^{\prime}, M^{\prime}\right)$ com $G, G^{\prime}$ grupos, $M$ um $\mathbb{Z}_{2} G$-módulo e $M^{\prime}$ um $\mathbb{Z}_{2} G^{\prime}$-módulo.

Uma aplicação de $(G, M)$ em $\left(G^{\prime}, M^{\prime}\right)$ é um par $(\alpha, \varphi)$ satisfazendo:

(i) $\alpha: G \rightarrow G^{\prime}$ um homomorfismo de grupos.

(ii) $\varphi: M^{\prime} \rightarrow M$ um homomorfismo de grupos abelianos tal que $\varphi\left(\alpha(g) \cdot m^{\prime}\right)=g \cdot \varphi\left(m^{\prime}\right), \forall g \in$ $G$ e $\forall m^{\prime} \in M^{\prime}$, isto é, $\varphi$ é uma aplicação de $\mathbb{Z}_{2} G$-módulos considerando $M^{\prime}$ como $\mathbb{Z}_{2} G$-módulo por restrição de escalares via $\alpha: g * m^{\prime}=\alpha(g) . m^{\prime}, \forall m^{\prime} \in M^{\prime}$.

Se $\alpha$ e $\varphi$ são isomorfismos, dizemos que $(G, M)$ e $\left(G^{\prime}, M^{\prime}\right)$ são isomorfos e denotamos $(G, M) \simeq\left(G^{\prime}, M^{\prime}\right)$.

\section{Aplicação Induzida em Cohomologia e $H^{*}(-;-)$ como funtor}

Seja $C$ a categoria cujos os objetos são pares $(G, M)$, com $G$ um grupo e $M$ um $\mathbb{Z}_{2} G$ módulo, e os morfismos são aplicações $(\alpha, \varphi)$ de $(G, M)$ em $\left(G^{\prime}, M^{\prime}\right)$ como acima. Dada uma aplicação $(\alpha, \varphi):(G, M) \rightarrow\left(G^{\prime}, M^{\prime}\right)$, consideremos $\epsilon: F \rightarrow \mathbb{Z}_{2}$ uma resolução projetiva de $\mathbb{Z}_{2}$ sobre $\mathbb{Z}_{2} G$ e $\epsilon^{\prime}: F^{\prime} \rightarrow \mathbb{Z}_{2}$ uma resolução projetiva de $\mathbb{Z}_{2}$ sobre $\mathbb{Z}_{2} G^{\prime}$.

Temos que $\epsilon^{\prime}: F^{\prime} \rightarrow \mathbb{Z}_{2}$ também é uma resolução projetiva de $\mathbb{Z}_{2}$ sobre $\mathbb{Z}_{2} G$ (via $\alpha$ ), pois cada $F_{n}^{\prime}$ pode ser considerado um $\mathbb{Z}_{2} G$-módulo com a $G$-ação

$$
\begin{aligned}
*: G \times F_{n}^{\prime} & \rightarrow F_{n}^{\prime} \\
(g, y) & \mapsto g * y:=\alpha(g) . y
\end{aligned}
$$

Isto é, $F_{n}^{\prime}$ é visto como $\mathbb{Z}_{2} G$-módulo por restrição de escalares considerando o homomorfismo (que também denotaremos por $\alpha$ ) $\alpha: \mathbb{Z}_{2} G \rightarrow \mathbb{Z}_{2} G^{\prime}$ tal que associa a cada $g \in \mathbb{Z}_{2} G$ 
o elemento $\alpha(g)$ e estendido linearmente. Daí, pela Proposição 1.3.1 existe uma aplicação $\tau: F \rightarrow F^{\prime}$, única a menos de homotopia. Temos que, $\tau(g \cdot x)=g \cdot \tau(x), \forall g \in G$ e $\forall x \in F$ (pois $\tau$ é uma aplicação de $\mathbb{Z}_{2} G$-módulos).

Assim, podemos considerar a aplicação

$$
\begin{aligned}
\operatorname{Hom}(\tau, \varphi): \operatorname{Hom}_{\mathbb{Z}_{2} G^{\prime}}\left(F^{\prime}, M^{\prime}\right) & \rightarrow \operatorname{Hom}_{\mathbb{Z}_{2} G}(F, M) \\
f & \mapsto \varphi \circ f \circ \tau
\end{aligned}
$$

a qual é uma aplicação de cadeias que induz uma aplicação bem definida, que vamos denotar por $(\alpha, \varphi)^{*}$,

$$
\begin{aligned}
(\alpha, \varphi)^{*}=(\operatorname{Hom}(\tau, \varphi))^{*}: H^{*}\left(G^{\prime} ; M^{\prime}\right) & \rightarrow H^{*}(G ; M) \\
{[f] } & \mapsto[\varphi \circ f \circ \tau]
\end{aligned}
$$

Em particular, considerando $G=G^{\prime}, \alpha=i d_{G}: G \rightarrow G$ e $\varphi: M^{\prime} \rightarrow M$ obtemos

$$
\begin{aligned}
\left(i d_{G}, \varphi\right)^{*}: H^{*}\left(G ; M^{\prime}\right) & \rightarrow H^{*}(G ; M) \\
{[f] } & \mapsto[\varphi \circ f] .
\end{aligned}
$$

Ainda se considerarmos $\alpha: G \rightarrow G^{\prime}$ e $\varphi=i d_{M}: M \rightarrow M$, temos

$$
\begin{aligned}
\left(\alpha, i d_{M}\right)^{*}: H^{*}\left(G^{\prime} ; M\right) & \rightarrow H^{*}(G ; M) \\
{[f] } & \mapsto[f \circ \tau] .
\end{aligned}
$$

Por simplicidade de notação, estamos usando o mesmo símbolo [ ] para representar classes em grupos diferentes.

A aplicação induzida em cohomologia $(\alpha, \varphi)^{*}$ satisfaz as seguintes propriedades:

(a) Se $\alpha=i d_{G}: G \rightarrow G$ e $\varphi=i d_{M}: M \rightarrow M$ então $(\alpha, \varphi)^{*}: i d_{H^{*}(G, M)}$

(b) Dadas duas aplicações de pares

$$
\begin{aligned}
& (\alpha, \varphi):(G, M) \rightarrow\left(G^{\prime}, M^{\prime}\right) \text { e }\left(\alpha^{\prime}, \varphi^{\prime}\right):\left(G^{\prime}, M^{\prime}\right) \rightarrow\left(G^{\prime \prime}, M^{\prime \prime}\right), \operatorname{com} G \stackrel{\alpha}{\rightarrow} G^{\prime} \stackrel{\alpha^{\prime}}{\rightarrow} G^{\prime \prime} \mathrm{e} \\
& M^{\prime \prime} \stackrel{\varphi^{\prime}}{\rightarrow} M^{\prime} \stackrel{\varphi}{\rightarrow} M, \text { tem-se: } \\
& (\alpha, \varphi)^{*} \circ\left(\alpha^{\prime}, \varphi^{\prime}\right)^{*}=\left(\alpha^{\prime} \circ \alpha, \varphi \circ \varphi^{\prime}\right)^{*} \stackrel{\text { not. }}{=}\left(\left(\alpha^{\prime}, \varphi^{\prime}\right) \circ(\alpha, \varphi)\right)^{*} .
\end{aligned}
$$

Deste modo $H^{*}(-;-)$ é um funtor contravariante na categoria $C, H^{*}(G ;-)$ é um funtor covariante do módulo coeficiente e $H^{*}(-; M)$ é um funtor contravariante.

Notações especiais:

1. $\alpha^{*}=\left(\alpha, i d_{M}\right)^{*}$. 
2. $\left(i d_{G}, \varphi\right)^{*} \stackrel{\text { not. }}{=} H^{*}(G ; \varphi)$ ou simplesmente $\varphi^{*}$ (quando não houver confusão).

Proposição 2.2.1. Se os pares $(G, M)$ e $\left(G^{\prime}, M^{\prime}\right)$ são isomorfos, isto é, $(G, M) \simeq$ $\left(G^{\prime}, M^{\prime}\right)$ então $H^{*}(G ; M) \simeq H^{*}\left(G^{\prime} ; M^{\prime}\right)$.

Demonstração: Por hipótese, $(G, M) \simeq\left(G^{\prime}, M^{\prime}\right)$, ou seja, existem isomorfismos $\alpha: G \rightarrow G^{\prime}$ e $\varphi: M^{\prime} \rightarrow M$. Sejam $\alpha^{-1}: G^{\prime} \rightarrow G$ e $\varphi^{-1}: M \rightarrow M^{\prime}$ os homomorfismos inversos de $\alpha$ e $\varphi$, respectivamente.

Consideremos $(\alpha, \varphi)^{*}: H^{*}\left(G^{\prime} ; M^{\prime}\right) \rightarrow H^{*}(G ; M)$. Como $(\alpha, \varphi)^{*} \circ\left(\alpha^{-1}, \varphi^{-1}\right)^{*}=$ $\left(\alpha^{-1} \circ \alpha, \varphi \circ \varphi^{-1}\right)^{*}=\left(i d_{G}, i d_{M}\right)^{*}=i d_{H^{*}(G ; M)}$ e $\left(\alpha^{-1}, \varphi^{-1}\right)^{*} \circ(\alpha, \varphi)^{*}=\left(\alpha \circ \beta^{-1}, \varphi^{-1} \circ \varphi\right)^{*}=$ $\left(i d_{G^{\prime}}, i d_{M^{\prime}}\right)^{*}=i d_{H^{*}\left(G^{\prime} ; M^{\prime}\right)}$ segue que $\left(\alpha^{-1}, \varphi^{-1}\right)^{*}$ é a aplicação inversa de $(\alpha, \varphi)^{*}$.

Logo, $H^{*}(G ; M) \simeq H^{*}\left(G^{\prime} ; M^{\prime}\right)$.

Corolário 2.2.1. Se $M$ e $M^{\prime}$ são isomorfos como $\mathbb{Z}_{2} G$-módulos então $H^{*}(G ; M) \simeq$ $H^{*}\left(G ; M^{\prime}\right)$.

Demonstração: Segue da proposição anterior, usando o fato de que $i d_{G}: G \rightarrow G$ é um isomorfismo.

Uma sequência exata curta de $\mathbb{Z}_{2} G$-módulos sempre induz uma sequência exata longa em homologia e cohomologia. Enunciaremos aqui apenas o resultado de interesse para nós, que é para cohomologia (ver [11], p.71).

Proposição 2.2.2. ([11], III, Proposição 6.1 (ii) p.71) Seja $0 \rightarrow M^{\prime} \stackrel{\varphi^{\prime}}{\rightarrow} M \stackrel{\varphi^{\prime \prime}}{\rightarrow} M^{\prime \prime} \rightarrow 0$ uma seqüência exata de $\mathbb{Z}_{2} G$-módulos. Então, $\forall n \in \mathbb{Z}$ existe uma aplicação natural $\delta^{n}: H^{n}\left(G, M^{\prime \prime}\right) \rightarrow H^{n+1}\left(G, M^{\prime}\right)$ tal que a seqüência $0 \longrightarrow H^{0}\left(G, M^{\prime}\right) \stackrel{\left(\varphi^{\prime}\right)^{0}}{\longrightarrow} H^{0}(G, M) \stackrel{\left(\varphi^{\prime \prime}\right)^{0}}{\longrightarrow} H^{0}\left(G, M^{\prime \prime}\right) \stackrel{\delta^{0}}{\longrightarrow} H^{1}\left(G, M^{\prime}\right) \stackrel{\left(\varphi^{\prime}\right)^{1}}{\longrightarrow} H^{1}(G, M) \longrightarrow \cdots$ é exata com as aplicações $\left(\varphi^{\prime}\right)^{n}$ e $\left(\varphi^{\prime \prime}\right)^{n}$ as induzidas em cohomologia, ou seja, $\left(\varphi^{\prime}\right)^{n}=$ $H^{n}\left(G, \varphi^{\prime}\right)$ e $\left(\varphi^{\prime \prime}\right)^{n}=H^{n}\left(G, \varphi^{\prime \prime}\right)$. A naturalidade do homomorfismo conexão significa que para qualquer diagrama comutativo

com linhas exatas, o quadrado
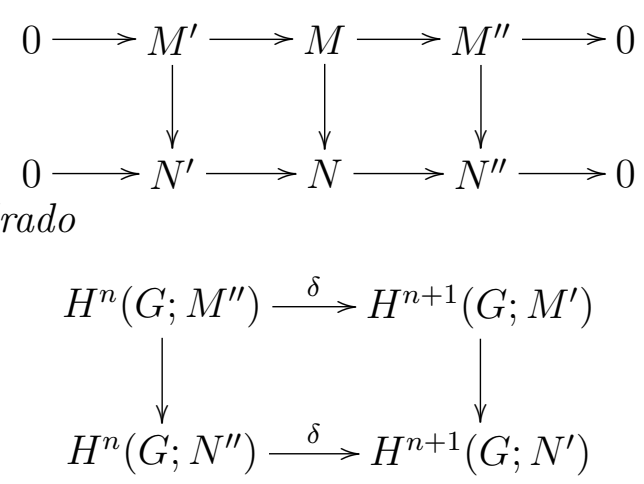

é comutativo. 


\subsubsection{Aplicação Restrição}

Sejam $G$ um grupo, $H$ um subgrupo de $G$ e $M$ um $\mathbb{Z}_{2} G$-módulo. Vimos que podemos ver $M$ como um $\mathbb{Z}_{2} H$-módulo por restrição de escalares que denotamos por $\operatorname{res}_{H}^{G} M$.

Sejam $\epsilon: F \rightarrow R$ e $\epsilon^{\prime}: F^{\prime} \rightarrow R$ resoluções projetivas de $\mathbb{Z}_{2} H$ e $\mathbb{Z} G$, respectivamente. Considerando os pares $\left(H, r e s_{H}^{G} M\right)$ e $(G, M)$, e a aplicação $\left(\alpha, i d_{M}\right)$, onde $\alpha: H \rightarrow G$ e $i d_{M}: M \rightarrow r e s_{H}^{G} M=M$ (como conjunto) é a aplicação identidade, obtemos a seguinte aplicação induzida em cohomologia:

$$
\begin{aligned}
r e s_{H, M}^{G} \stackrel{\text { not. }}{=}\left(\alpha, i d_{M}\right)^{*}: H^{*}(G ; M) & \rightarrow H^{*}(H ; M)=H^{*}\left(H, \operatorname{Res}_{H}^{G} M\right) \\
{[f] } & \mapsto[i d \circ f \circ \tau]
\end{aligned}
$$

Obviamente, $\epsilon^{\prime}: F^{\prime} \rightarrow R$ também é uma resolução projetiva de $R$ sobre $R H$ e como os grupos de cohomologia independem da resolução, podemos tomar $F=F^{\prime}$ e consequentemente, $\tau: F \mapsto F^{\prime}$ como sendo a identidade. Logo teremos que

$$
\operatorname{res}_{H, M}^{G}([f])=[i d \circ f \circ \tau]=[f]
$$

com $f$ visto no primeiro membro como uma aplicação de $\mathbb{Z}_{2} G$-módulos e no segundo membro como uma aplicação de $\mathbb{Z}_{2} H$-módulos. Tal aplicação é denominada aplicação restrição em geral e a denotaremos simplesmente por $r e s_{H}^{G}$ (sem mencionar o módulo $M)$.

Observação 2.2.1. Similarmente, define-se "aplicação induzida em homologia" e resultados análogos aos apresentados para cohomologia são obtido para homologia.

Proposição 2.2.3. (Lema de Shapiro, [11], Proposição 6.2, p.73 ) Sejam G um grupo, $H$ um subgrupo de $G$ e $M$ um $\mathbb{Z}_{2} H$-módulo. Temos os seguintes isomorfismos:

$$
\begin{aligned}
& \text { 1. } H_{*}(H ; M) \simeq H_{*}\left(G ; \operatorname{Ind}_{H}^{G} M\right) . \\
& \text { 2. } H^{*}(H ; M) \simeq H^{*}\left(G ; \operatorname{Coind}_{H}^{G} M\right) \text {. }
\end{aligned}
$$

Corolário 2.2.2. Se $[G: H]<\infty$ ent $\tilde{a} o H^{*}\left(H ; \mathbb{Z}_{2} H\right) \simeq H^{*}\left(G ; \mathbb{Z}_{2} G\right)$.

Demonstração: $H^{*}\left(H ; \mathbb{Z}_{2} H\right) \stackrel{\text { Shapiro }}{\simeq} H^{*}\left(G ; \operatorname{Coind}_{H}^{G} \mathbb{Z}_{2} H\right) \stackrel{[G: H]<\infty}{\simeq} H^{*}\left(G ; \operatorname{Ind}_{H}^{G} \mathbb{Z}_{2} H\right) \simeq$ $H^{*}\left(G ; \mathbb{Z}_{2} G \otimes_{\mathbb{Z}_{2} H} \mathbb{Z}_{2} H\right) \simeq H^{*}\left(G ; \mathbb{Z}_{2} G\right)$.

\subsection{Grupos de (Co)homologia Relativa}

Nesta seção apresentamos o conceito de (co)homologia relativa para um grupo $G$ e uma família $\mathcal{S}$ de subgrupos de $G$. Tal conceito foi introduzido por Bieri e Eckmann em [10], 
considerando o anel $\mathbb{Z}$ com coeficientes em um $\mathbb{Z} G$-módulo $M$, mas aqui definiremos sobre o corpo $\mathbb{Z}_{2}$ com coeficientes em um $\mathbb{Z}_{2} G$-módulo $M$ (como Kropholler e Roller em [17]). O principal resultado desta seção é o Teorema da Sequência Exata Longa em cohomologia (para par), e cuja a prova apresentamos em detalhes.

Considere um par grupo $(G, \mathcal{S})$ consistindo de um grupo $G$ e uma família $\mathcal{S}=\left(S_{i}\right)_{i \in I}$ de subgrupos de $G$ (não necessariamente distintos). Seja $\mathbb{Z}_{2}(G / \mathcal{S})$ o $\mathbb{Z}_{2}$-módulo livre gerado pelas classes $g S_{i}$, sobre o qual $G$ atua por multiplicação à esquerda e considere a aplicação aumentação usual $\varepsilon$, a que leva cada gerador $g S_{i}$ em $1 \in \mathbb{Z}_{2}$, e denote por $\Delta$ seu núcleo.

\section{Observação 2.3.1.}

1. Note que $\mathbb{Z}_{2}(G / \mathcal{S})=\bigoplus_{i \in I} \mathbb{Z}_{2}\left(G / S_{i}\right)=\bigoplus_{i \in I} \operatorname{Ind}_{S_{i}}^{G} \mathbb{Z}_{2}=\bigoplus_{i \in I}\left(\mathbb{Z}_{2} G \otimes_{\mathbb{Z}_{2} S_{i}} \mathbb{Z}_{2}\right)$.

2. Observe que $\varepsilon$ e $\epsilon$ indicam homomorfismos distintos (aplicações aumentação), mais precisamente $\varepsilon: \mathbb{Z}_{2}(G / \mathcal{S}) \rightarrow \mathbb{Z}_{2}$ e $\epsilon: \mathbb{Z}_{2} G \rightarrow \mathbb{Z}_{2}$ são homomorfismos com dominios diferentes.

Com isso temos a seguinte definição:

Definição 2.3.1. Os grupos de (co)homologia relativa para o par grupo $(G, \mathcal{S})$ com coeficientes em um $\mathbb{Z}_{2} G$-módulo à esquerda $M$ são definidos, para todo inteiro $i \geq 1$, por:

$$
\begin{aligned}
H^{i}(G, \mathcal{S} ; M) & =H^{i-1}\left(G ; \operatorname{Hom}_{\mathbb{Z}_{2}}(\Delta, M)\right) e \\
H_{i}(G, \mathcal{S} ; M) & =H_{i-1}\left(G ; \Delta \otimes_{\mathbb{Z}_{2}} M\right) .
\end{aligned}
$$

\section{Observação 2.3.2.}

1. Segue da definição que $H^{i}(G, \mathcal{S} ; M)=H_{i}(G, \mathcal{S} ; M)=0$, para $i \leq 0$.

2. Por convenção, para $\mathcal{S}=\emptyset$, considera-se $H^{i}(G, \emptyset ; M):=H^{i}(G ; M)$ e $H_{i}(G, \emptyset ; M):=$ $H_{i}(G ; M)$.

3. Se a família $\mathcal{S}$ tem apenas um subgrupo, ou seja $\mathcal{S}=\{S\}$, denota-se $H^{n}(G, \mathcal{S} ; M)=$ $H^{n}(G, S ; M)$ e $H_{n}(G, \mathcal{S} ; M)=H_{n}(G, S ; M)$.

4. Também é usual denotar, para cada família $\mathcal{S}=\left(S_{j}\right)_{j \in J}$ de subgrupos de $G$,

$$
H_{i}(\mathcal{S} ; M):=\bigoplus_{j \in J} H_{i}\left(S_{j} ; M\right) \text { e } H^{i}(\mathcal{S} ; M):=\prod_{j \in J} H^{i}\left(S_{j} ; M\right) .
$$


5. Como para (co)homologia absoluta pode-se verificar que $H^{*}(G, \mathcal{S} ; M)$ é um funtor das duas variáveis $((G, \mathcal{S}), M)$. Mais precisamente, se $\mathcal{C}$ é a categoria cujos objetos são pares $((G, \mathcal{S}), M)$, onde $(G, \mathcal{S})$ é um par grupo $\left(\mathcal{S}=\left\{S_{i}, i \in I\right\}\right.$, uma família de subgrupos de $G$ ) e $M$ é um $\mathbb{Z}_{2} G$-módulo, e cujos morfismos são as aplicações $\psi:\left(\left(G, \mathcal{S}=\left\{S_{i}, i \in I\right\}\right), M\right) \rightarrow\left(\left(L, \mathcal{R}=\left\{R_{j}, j \in J\right\}\right), N\right)$ consistindo de

(a) um homomorfismo $\alpha: G \rightarrow L$,

(b) uma aplicação $\pi: I \rightarrow J$ tal que $\alpha\left(S_{i}\right) \subset R_{\pi(i)}$,

(c) uma aplicação $\phi: N \rightarrow M$ tal que $\phi(\alpha(g) . n)=g . \phi(n)$, i.e., $\phi$ é um homomorfismo de $\mathbb{Z}_{2} G$-módulos via $\alpha$.

então existe (vide [4] Teorema 2.1), um homomorfismo $\psi^{*}: H^{*}(L, \mathcal{R} ; N) \rightarrow$ $H^{*}(G, \mathcal{S} ; M)$ (induzido por $\left.\psi\right)$, satisfazendo as seguintes condições:

- se $\psi \quad: \quad((G, \mathcal{S}), M) \rightarrow((G, \mathcal{S}), M)$ é a aplicação identidade, então $\psi^{*}: H^{*}(G, \mathcal{S} ; M) \rightarrow H^{*}(G, \mathcal{S} ; M)$ é o homomorfismo identidade.

- Se $\psi:((G, \mathcal{S}), M) \rightarrow((L, \mathcal{R}), N)$ e $\gamma:((L, \mathcal{R}), N) \rightarrow((K, \mathcal{O}), P)$ são homomorfismos, então $(\gamma \circ \psi)^{*}=\psi^{*} \circ \gamma^{*}$, de modo que $H^{*}((-,-) ;-)$ é um funtor contravariante em $\mathcal{C}$.

Um resultado similar vale para homologia relativa.

Teorema 2.3.1. ([10], Proposição 1.1, p.281 ou [12], Proposição 2.5.6) $\operatorname{Sejam~}(G, \mathcal{S})$ um par grupo com $\mathcal{S}=\left(S_{i}\right)_{i \in I}$ e $M$ um $\mathbb{Z}_{2} G$-módulo. Então temos a seguinte sequência exata longa

$\left.0 \quad \rightarrow \quad H^{0}(G ; M) \quad \rightarrow \quad \prod_{i \in I} H^{0}\left(S_{i} ; M\right)\right) \quad \stackrel{\delta}{\rightarrow} H^{1}(G, \mathcal{S} ; M) \quad \stackrel{J}{\rightarrow} H^{1}(G ; M) \stackrel{\text { res }}{\rightarrow}$ $\stackrel{r^{e s} G}{\rightarrow} \prod_{i \in I} H^{1}\left(S_{i} ; M\right) \rightarrow \cdots$,

que é natural no $\mathbb{Z}_{2} G$-módulo $M$ e no par grupo $(G, \mathcal{S})$.

Demonstração: Considere a seguinte sequência exata curta de $\mathbb{Z}_{2} G$-módulos

$$
0 \longrightarrow \Delta \stackrel{i}{\longrightarrow} \mathbb{Z}_{2}(G / \mathcal{S}) \stackrel{\varepsilon}{\longrightarrow} \mathbb{Z}_{2} \longrightarrow 0
$$

onde $i$ é a aplicação inclusão, $\varepsilon$ a aplicação aumentação usual já definida. 
Note que $\mathbb{Z}_{2}$ é $\mathbb{Z}_{2}$-livre, logo a sequência (6.3) cinde ([22], Exercício 3.7), e como uma consequência desse fato temos que a seguinte sequência,

$$
0 \rightarrow \operatorname{Hom}_{\mathbb{Z}_{2}}\left(\mathbb{Z}_{2}, M\right) \stackrel{\varepsilon^{\#}}{\rightarrow} \operatorname{Hom}_{\mathbb{Z}_{2}}\left(\mathbb{Z}_{2}(G / \mathcal{S}), M\right) \stackrel{i \#}{\rightarrow} \operatorname{Hom}_{\mathbb{Z}_{2}}(\Delta, M) \rightarrow 0
$$

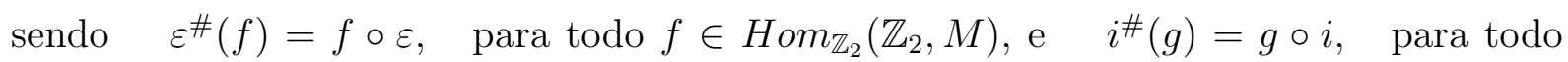
$g \in \operatorname{Hom}_{\mathbb{Z}_{2}}\left(\mathbb{Z}_{2}(G / \mathcal{S}), M\right)$, é também exata e cinde. Notemos que $i^{\#}(g)=\left.g\right|_{\Delta}: \Delta \rightarrow M$ (a aplicação restrição).

Considere agora uma resolução livre de $\mathbb{Z}_{2}$ sobre $\mathbb{Z}_{2} G$

$$
F: \quad \cdots \longrightarrow F_{n} \stackrel{\partial_{n}}{\longrightarrow} \cdots \stackrel{\partial_{2}}{\longrightarrow} F_{1} \stackrel{\partial_{1}}{\longrightarrow} F_{0}=\mathbb{Z}_{2} G \stackrel{\epsilon}{\longrightarrow} \mathbb{Z}_{2} \longrightarrow 0
$$

Como cada $\mathbb{Z}_{2} G$-módulo $F_{n}$ é livre, logo projetivo, pelo Teorema 1.4.6, temos que a sequência

$$
\begin{aligned}
0 \rightarrow \operatorname{Hom}_{\mathbb{Z}_{2} G}\left(F_{n}, \operatorname{Hom}_{\mathbb{Z}_{2}}\left(\mathbb{Z}_{2}, M\right)\right) \stackrel{\tilde{\varepsilon}_{n}^{\#}}{\rightarrow} \operatorname{Hom}_{\mathbb{Z}_{2} G}\left(F_{n}, \operatorname{Hom}_{\mathbb{Z}_{2}}\left(\mathbb{Z}_{2}(G / \mathcal{S}), M\right)\right) \stackrel{\widetilde{i}_{n}^{\#}}{\rightarrow} \operatorname{Hom}_{\mathbb{Z}_{2} G}\left(F_{n}, \operatorname{Hom}_{\mathbb{Z}_{2}}(\Delta, M)\right) \rightarrow 0,
\end{aligned}
$$

é uma sequência exata curta de $\mathbb{Z}_{2} G$-módulos, para todo $n$, onde os homomorfismos induzidos $\widetilde{\epsilon_{n}}{ }^{\#}$ e ${\widetilde{i_{n}}}^{\#}$ são tais que,

$$
\begin{aligned}
& {\widetilde{\varepsilon_{n}}}^{\#}(f)=\varepsilon^{\#} \circ f, \forall f \in \operatorname{Hom}_{\mathbb{Z}_{2} G}\left(F_{n}, H^{\# o m} \mathbb{Z}_{2}\left(\mathbb{Z}_{2}, M\right)\right), \\
& \widetilde{i_{n}}(g)=i^{\#} \circ g, \forall g \in \operatorname{Hom}_{\mathbb{Z}_{2} G}\left(F_{n}, H_{\mathbb{Z}_{2}}\left(\mathbb{Z}_{2}(G / \mathcal{S}), M\right) .\right.
\end{aligned}
$$

Obtemos então a sequência exata curta de complexos de cocadeias,

$$
\begin{aligned}
& 0 \rightarrow \operatorname{Hom}_{\mathbb{Z}_{2} G}\left(F, H_{0 m}\left(\mathbb{Z}_{2}, M\right)\right) \stackrel{\tilde{\varepsilon}_{n}^{\#}}{\longrightarrow} H_{\mathbb{Z}_{2}} \mathbb{Z}_{2} G\left(F, H_{\mathbb{Z}_{2}}\left(\mathbb{Z}_{2}(G / \mathcal{S}), M\right)\right) \stackrel{\tilde{i}_{n}^{\#}}{\longrightarrow} \\
& \stackrel{\widetilde{i}_{n}^{\#}}{\rightarrow} H_{o m} \mathbb{Z}_{2} G\left(F, H_{0 m} \mathbb{Z}_{2}(\Delta, M)\right) \rightarrow 0,
\end{aligned}
$$

mais precisamente, denotando, para todo $n \in \mathbb{Z}, n \geq 0$,

$$
\begin{aligned}
& C^{n}=\operatorname{Hom}_{\mathbb{Z}_{2} G}\left(F_{n}, \operatorname{Hom}_{\mathbb{Z}_{2}}\left(\mathbb{Z}_{2}, M\right)\right), \\
& D^{n}=\operatorname{Hom}_{\mathbb{Z}_{2} G}\left(F_{n}, \operatorname{Hom}_{\mathbb{Z}_{2}}\left(\mathbb{Z}_{2}(G / \mathcal{S}), M\right)\right) \mathrm{e} \\
& E^{n}=\operatorname{Hom}_{\mathbb{Z}_{2} G}\left(F_{n}, \operatorname{Hom}_{\mathbb{Z}_{2}}(\Delta, M)\right),
\end{aligned}
$$


obtemos o seguinte diagrama:

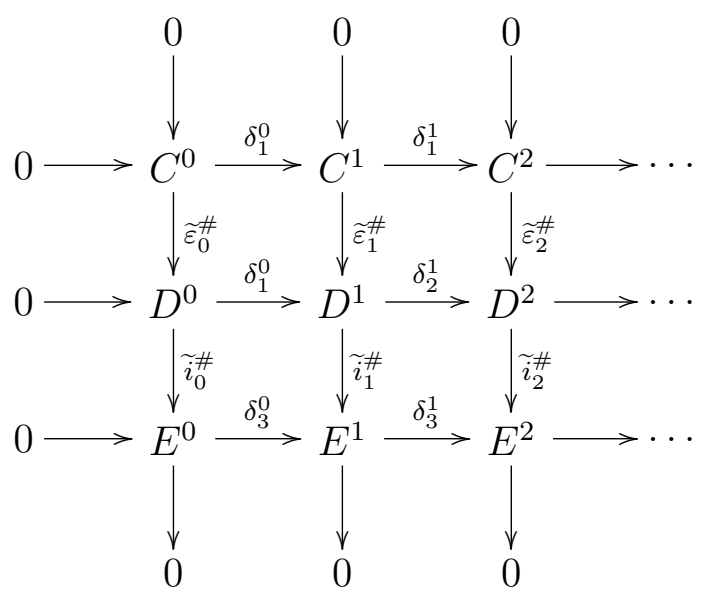

Assim a sequência exata curta de complexos de cocadeias

$$
0 \longrightarrow C \stackrel{\widetilde{\varepsilon}^{\#}}{\longrightarrow} D \stackrel{\widetilde{i}^{\#}}{\longrightarrow} E \longrightarrow 0
$$

onde $C=\left(C^{n}\right)=\left(H_{\mathrm{O}_{2} G}\left(F_{n}, H_{\mathbb{Z}_{2}} \mathbb{Z}_{2}\left(\mathbb{Z}_{2}, M\right)\right)\right)$,

$D=\left(D^{n}\right)=\left(H_{o m} \mathbb{Z}_{2} G\left(F_{n}, H_{o m} \mathbb{Z}_{2}\left(\mathbb{Z}_{2}(G / \mathcal{S}), M\right)\right)\right) \mathrm{e}$

$E=\left(E^{n}\right)=\left(\operatorname{Hom}_{\mathbb{Z}_{2} G}\left(F_{n}, H_{o m} \mathbb{Z}_{2}(\Delta, M)\right)\right)$

induz, pelo Teorema 1.1.1, a seguinte sequência exata longa em cohomologia :

$$
\begin{aligned}
& 0 \rightarrow H^{0}\left(H o m_{\mathbb{Z}_{2} G}\left(F, H_{o m} \mathbb{Z}_{2}\left(\mathbb{Z}_{2}, M\right)\right)\right) \stackrel{\varepsilon_{0}^{*}}{\rightarrow} H^{0}\left(\operatorname{Hom}_{\mathbb{Z}_{2} G}\left(F, H o m_{\mathbb{Z}_{2}}\left(\mathbb{Z}_{2}(G / \mathcal{S}), M\right)\right)\right) \stackrel{i_{0}^{*}}{\rightarrow} \\
& \stackrel{i_{0}^{*}}{\rightarrow} H^{0}\left(\operatorname{Hom}_{\mathbb{Z}_{2} G}\left(F, H_{\mathbb{Z}_{2}}(\Delta, M)\right)\right) \stackrel{\rho^{0}}{\rightarrow} H^{1}\left(\operatorname{Hom}_{\mathbb{Z}_{2} G}\left(F, H_{\mathbb{Z}_{2}}\left(\mathbb{Z}_{2}, M\right)\right)\right) \rightarrow \cdots,
\end{aligned}
$$

onde $\varepsilon_{n}^{*}([f])=\left[\widetilde{\varepsilon}_{n}^{\#}(f)\right]=\left[\varepsilon^{\#} \circ f\right], f \in H^{n}\left(\operatorname{Hom}_{\mathbb{Z}_{2} G}\left(F, H_{o m} m_{\mathbb{Z}_{2} G}\left(\mathbb{Z}_{2}, M\right)\right)\right), \quad i_{n}^{*}([g])=$ $\left.\widetilde{i_{n}^{\#}}(g)\right]=\left[i^{\#} \circ g\right], g \in H^{n}\left(\operatorname{Hom}_{\mathbb{Z}_{2} G}\left(F, H_{o m} \mathbb{Z}_{2}\left(\mathbb{Z}_{2}(G / \mathcal{S}), M\right)\right)\right)$ e $\quad \rho^{n}$ é o homomorfismo conectante.

Observemos agora que

- $H^{n}\left(H o m_{\mathbb{Z}_{2} G}\left(F, H o m_{\mathbb{Z}_{2}}\left(\mathbb{Z}_{2}, M\right)\right)\right):=H^{n}\left(G ; H o m_{\mathbb{Z}_{2}}\left(\mathbb{Z}_{2}, M\right)\right)$,

- $H^{n}\left(H_{o m} \mathbb{Z}_{2} G\left(F, H o m_{\mathbb{Z}_{2}}\left(\mathbb{Z}_{2}(G / \mathcal{S}), M\right)\right)\right):=H^{n}\left(G ; H o m_{\mathbb{Z}_{2}}\left(\mathbb{Z}_{2}(G / \mathcal{S}), M\right)\right)$ e

- $H^{n}\left(\operatorname{Hom}_{\mathbb{Z}_{2} G}\left(F, H_{o m} \mathbb{Z}_{2}(\Delta, M)\right)\right):=H^{n}\left(G ; \operatorname{Hom}_{\mathbb{Z}_{2}}(\Delta, M)\right)$.

Deste modo, a sequência (2.4) equivale a seguinte sequência exata (de fato essa é a menos de isomorfismo a sequência desejada): 


$$
\begin{aligned}
& 0 \rightarrow H^{0}\left(G ; H o m_{\mathbb{Z}_{2}}\left(\mathbb{Z}_{2}, M\right)\right) \stackrel{\varepsilon_{0}^{*}}{\rightarrow} H^{0}\left(G ; H o m_{\mathbb{Z}_{2}}\left(\mathbb{Z}_{2}(G / \mathcal{S}), M\right)\right) \stackrel{i_{0}^{*}}{\rightarrow} H^{0}\left(G ; H o m_{\mathbb{Z}_{2}}(\Delta, M)\right) \stackrel{\rho^{0}}{\rightarrow} \\
& \stackrel{\rho^{0}}{\rightarrow} H^{1}\left(G ; H o m_{\mathbb{Z}_{2}}\left(\mathbb{Z}_{2}, M\right)\right) \rightarrow \cdots(2.5)
\end{aligned}
$$

Temos ainda que

1. $H^{n}\left(G ; H_{o m} \mathbb{Z}_{2}\left(\mathbb{Z}_{2}, M\right)\right) \stackrel{\varphi *}{\simeq} H^{n}(G ; M)$, onde $M \stackrel{\varphi}{\simeq} H_{o m} \mathbb{Z}_{2}\left(\mathbb{Z}_{2}, M\right)$ (vide $1.4 .1 \mathrm{e}$ Corolário 2.2.1), sendo $\varphi^{*}$ o homomorfismo induzido de $\varphi$.

2. $H^{n}\left(G ; H o m_{\mathbb{Z}_{2}}(\Delta, M)\right):=H^{n+1}(G, \mathcal{S} ; M)$

3. $H^{n}\left(G ; H_{\text {om }_{2}}\left(\mathbb{Z}_{2}(G / \mathcal{S}), M\right)\right) \stackrel{\eta}{\simeq} \prod_{i \in I} H^{n}\left(S_{i} ; M\right)$. Vamos exibir esse isomorfismo $\eta$. Temos

$H \operatorname{Hom}_{\mathbb{Z}_{2}}\left(\mathbb{Z}_{2}(G / \mathcal{S}), M\right)=H o m_{\mathbb{Z}_{2}}\left(\bigoplus_{i \in I} \mathbb{Z}_{2}\left(G / S_{i}\right), M\right) \stackrel{\phi}{\simeq} \prod_{i \in I} H o_{\mathbb{Z}_{2}}\left(\mathbb{Z}_{2}\left(G / S_{i}\right), M\right)$, com

$$
\begin{aligned}
\phi: \operatorname{Hom}_{\mathbb{Z}_{2}}\left(\bigoplus_{i \in I} \mathbb{Z}_{2}\left(G / S_{i}\right), M\right) & \rightarrow \prod_{k \in I} \text { Hom }_{\mathbb{Z}_{2}}\left(\mathbb{Z}_{2}\left(G / S_{k}\right), M\right) \\
f & \mapsto\left(f \circ j_{k}\right)_{k \in I}
\end{aligned}
$$

sendo $j_{k}: \mathbb{Z}_{2}\left(G / S_{k}\right) \rightarrow \bigoplus_{i \in I} \mathbb{Z}_{2}\left(G / S_{i}\right)$ a inclusão. Assim temos:

- $H^{n}\left(G ; H o m_{\mathbb{Z}_{2}}\left(\mathbb{Z}_{2}(G / S), M\right)\right) \stackrel{\phi^{*}}{\simeq} H^{n}\left(G ; \prod_{i \in I} H_{i \in m_{\mathbb{Z}_{2}}}\left(\mathbb{Z}_{2}\left(G / S_{i}\right), M\right)\right)$ onde $\phi^{*}$ é o homomorfismo induzido de $\phi$, isto é,

$$
\begin{aligned}
\phi^{*}: H^{n}\left(G ; H_{o m} \mathbb{Z}_{2}\left(\mathbb{Z}_{2}(G / S), M\right)\right) & \rightarrow H^{n}\left(G ; \prod_{i \in I} H_{\text {om }}\left(\mathbb{Z}_{2}\left(G / S_{i}\right), M\right)\right) \\
{[h] } & \mapsto \phi^{*}([h])=[\phi \circ h] .
\end{aligned}
$$

- $H^{n}\left(G ; \prod_{i \in I} H_{o m} m_{\mathbb{Z}_{2}}\left(\mathbb{Z}_{2}\left(G / S_{i}\right), M\right)\right) \stackrel{\gamma}{\simeq} \prod_{i \in I} H^{n}\left(G ; H o m_{\mathbb{Z}_{2}}\left(\mathbb{Z}_{2}\left(G / S_{i}\right), M\right)\right)$, com

$$
\begin{aligned}
\gamma: H^{n}\left(G ; \prod_{i \in I} \operatorname{Hom}_{\mathbb{Z}_{2}}\left(\mathbb{Z}_{2}\left(G / S_{i}\right), M\right)\right) & \rightarrow \prod_{i \in I} H^{n}\left(G ; \operatorname{Hom}_{\mathbb{Z}_{2}}\left(\mathbb{Z}_{2}\left(G / S_{i}\right), M\right)\right), \\
{[f] } & \mapsto\left(\left[p_{i} \circ f\right]\right)_{i \in I}
\end{aligned}
$$

onde $p_{i}: \prod_{k \in I} \operatorname{Hom}_{\mathbb{Z}_{2}}\left(\mathbb{Z}_{2}\left(G / S_{k}\right), M\right), \rightarrow \operatorname{Hom}_{\mathbb{Z}_{2}}\left(\mathbb{Z}_{2}\left(G / S_{i}\right), M\right)$ é a projeção.

Considere agora as aplicações 
- $\psi *=\left(\psi_{i} *\right)_{i \in I}$, onde

$$
\begin{aligned}
\left(\psi_{i} *\right)_{i \in I}: \prod_{i \in I} H^{n}\left(G ; \operatorname{Hom}_{\mathbb{Z}_{2}}\left(\mathbb{Z}_{2}\left(G / S_{i}\right), M\right)\right) & \rightarrow \prod_{i \in I} H^{n}\left(G ; \operatorname{Coind}_{S_{i}}^{G} M\right) \\
\left(\left[f_{i}\right]\right)_{i \in I} & \mapsto\left(\psi_{i}^{*}\left[f_{i}\right]\right)_{i \in I}
\end{aligned}
$$

com $\psi_{i} *$ a induzida do isomorfismo

$$
\begin{aligned}
\psi_{i}: \operatorname{Hom}_{\mathbb{Z}_{2}}\left(\mathbb{Z}_{2}\left(G / S_{i}\right), M\right) & \rightarrow \operatorname{Coind}_{S_{i}}^{G} M \\
f_{i} & \mapsto \psi_{i}\left(f_{i}\right),
\end{aligned}
$$

sendo

$$
\begin{aligned}
\psi_{i}\left(f_{i}\right): \mathbb{Z}_{2} G & \rightarrow M \\
g & \mapsto g f_{i}\left(g^{-1} S_{i}\right) .
\end{aligned}
$$

- $\pi^{*}=\left(\pi_{i}^{*}\right)_{i \in I}: \prod_{i \in I} H^{n}\left(G ; \operatorname{Coind}_{S_{i}}^{G} M\right) \rightarrow \prod_{i \in I} H^{n}(G ; M)$, onde $\pi_{i}^{*}$ é a induzida em cohomologia de

$$
\begin{aligned}
\pi_{i}: \operatorname{Coind}_{S_{i}}^{G} M & \rightarrow M \\
f_{i} & \mapsto \pi_{i}\left(f_{i}\right)=f_{i}(1)
\end{aligned}
$$

sendo $1 \in \mathbb{Z}_{2} G$

- $\left(r e s_{i}\right)_{i \in I}$, tal que

$$
\begin{aligned}
\left(\operatorname{res}_{i}\right)_{i \in I}: \prod_{i \in I} H^{n}(G ; M) & \rightarrow \prod_{i \in I} H^{n}\left(S_{i} ; M\right) \\
\left(\left[f_{i}\right]\right)_{i \in I} & \mapsto\left(\operatorname{res}_{S_{i}}^{G}\left(\left[f_{i}\right]\right)\right)_{i \in I}
\end{aligned}
$$

Note que $\phi^{*}, \gamma, \psi^{*}$ e $\left(r e s_{i}\right)_{i \in I} \circ\left(\pi_{i}^{*}\right)_{i \in I}=\left(\text { res }_{i} \circ \pi_{i}^{*}\right)_{i \in I}$ são isomorfismos (de fato tem-se que $r e s_{i} \circ \pi_{i}^{*}$ é o isomorfismo de Shapiro) e portanto a composta

$$
\begin{aligned}
& H^{n}\left(G ; H o m_{\mathbb{Z}_{2}}\left(\mathbb{Z}_{2}\left(G / S_{i}\right), M\right)\right) \stackrel{\phi^{*}}{\rightarrow} H^{n}\left(G ; \prod_{i \in I} H o m_{\mathbb{Z}_{2}}\left(\mathbb{Z}_{2}\left(G / S_{i}\right), M\right)\right) \stackrel{\gamma}{\rightarrow} \\
& \stackrel{\gamma}{\rightarrow} \prod_{i \in I} H^{n}\left(G ; H_{\operatorname{lom}_{\mathbb{Z}_{2}}}\left(\mathbb{Z}_{2}\left(G / S_{i}\right), M\right)\right) \stackrel{\psi^{*}}{\rightarrow} \prod_{i \in I} H^{n}\left(G ; \operatorname{Coind}_{S_{i}}^{G} M\right) \stackrel{\pi^{*}}{\rightarrow} \prod_{i \in I} H^{n}(G ; M) \stackrel{\left(\text { res }_{i}\right)_{i \in I}}{\longrightarrow} \\
& \stackrel{\left(\text { res }_{i}\right)_{i \in I}}{\longrightarrow} \prod_{i \in I} H^{n}\left(S_{i} ; M\right),
\end{aligned}
$$

é um isomorfismo, o qual denotaremos por simplesmente por $\eta$ (ao invés de $\eta^{n}$ ou $\left.\eta^{*}\right)$, ou seja, $\eta=\left(\text { res }_{i}\right)_{i \in I} \circ\left(\pi_{i}^{*}\right)_{i \in I^{\circ}} \psi^{*} \circ \gamma \circ \phi^{*}$. Assim, $H^{n}\left(G ; \operatorname{Hom}_{\mathbb{Z}_{2}}\left(\mathbb{Z}_{2}(G / \mathcal{S}), M\right)\right) \stackrel{\eta}{\simeq}$ 


$$
\prod_{i \in I} H^{n}\left(S_{i} ; M\right)
$$

De 1., 2., 3. e, considerando as aplicações dadas pelas composições (vide diagramas) abaixo: $\quad\left(r e s_{\mathcal{S}}^{G}\right)^{n}:=\eta \circ \varepsilon_{n}^{*} \circ \varphi^{*}, \quad \delta^{n}:=i_{n}^{*} \circ \eta^{-1}$ e $\quad J^{n+1}:=\varphi^{*} \circ \rho^{n}$

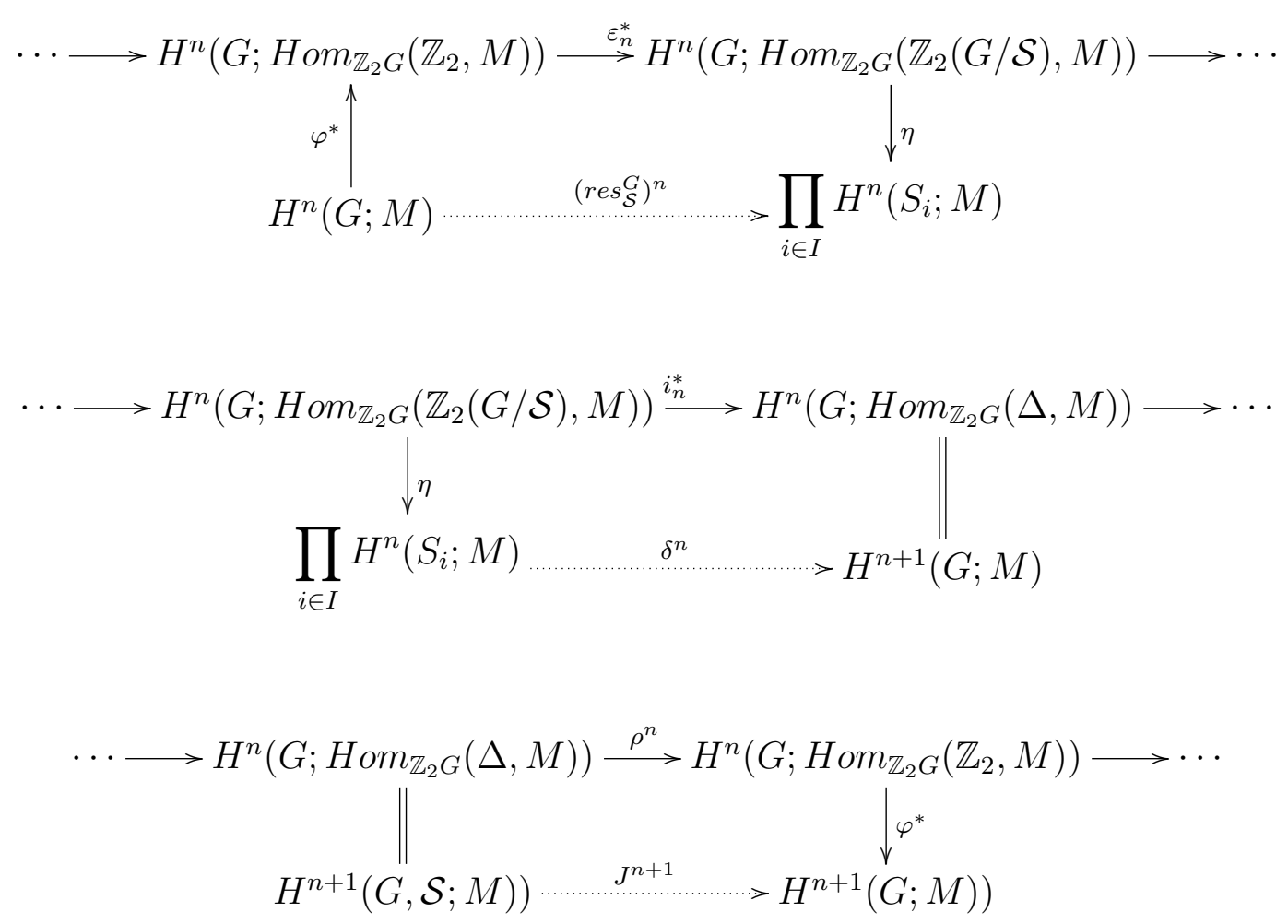

obtemos a sequência exata longa com os grupos de cohomologia desejados.

Verifiquemos que $\left(\eta \circ \varepsilon_{n}^{*} \circ \varphi^{*}\right)=\left(\operatorname{res}_{\mathcal{S}}^{G}\right)^{n}$, que denotaremos apenas por res $s_{\mathcal{S}}^{G}$ é de fato uma aplicação "restrição", mais precisamente, coincide com a aplicação abaixo:

$$
H^{n}(G ; M) \rightarrow \prod_{i \in I} H^{n}\left(S_{i} ; M\right) ;([f]) \mapsto\left(\operatorname{res}_{i}([f])\right)_{i \in I} \stackrel{\text { abuso-not. }}{=}([f])_{i \in I},
$$

para todo $[f] \in H^{n}(G ; M)$, onde res $_{i}$ indica $\operatorname{res}_{S_{i}}^{G}$.

De fato, seja $[f] \in H^{n}(G ; M)$. Temos que:

$\left(\eta \circ \varepsilon_{n}^{*} \circ \varphi^{*}\right)([f])=\left(\eta \circ \varepsilon_{n}^{*}\right)([\varphi \circ f])=\eta\left(\left[\varepsilon^{\#} \circ \varphi \circ f\right]\right)=\left(\left(r e s_{i}\right)_{i \in I} \circ\left(\pi_{i}^{*}\right)_{i \in I} \circ \psi^{*} \circ \gamma \circ \phi^{*}\right)\left(\left(\left[\varepsilon^{\#} \circ \varphi \circ\right.\right.\right.$ $f]))=\left(\left(r e s_{i}\right)_{i \in I} \circ\left(\pi_{i}^{*}\right)_{i \in I} \circ \psi^{*} \circ \gamma\right)\left(\left[\phi \circ \varepsilon^{\#} \circ \varphi \circ f\right]\right)=\left(\left(r e s_{i}\right)_{i \in I} \circ\left(\pi_{i}^{*}\right)_{i \in I} \circ \psi^{*}\right)\left(\left(\left[p_{i} \circ \phi \circ \varepsilon^{\#} \circ \varphi \circ\right.\right.\right.$ $\left.f])_{i \in I}\right)=\left(\left(r e s_{i}\right)_{i \in I} \circ \pi^{*}\right)\left(\left(\left[\psi_{i} \circ p_{i} \circ \phi \circ \varepsilon^{\#} \circ \varphi \circ f\right]\right)_{i \in I}\right)=\left(r e s_{i}\right)_{i \in I}\left(\left(\left[\pi_{i} \circ \psi_{i} \circ p_{i} \circ \phi \circ \varepsilon^{\#} \circ \varphi \circ f\right]\right)_{i \in I}\right)=$ $\left(\operatorname{res}_{i}\left(\left[\pi_{i} \circ \psi_{i} \circ p_{i} \circ \phi \circ \varepsilon^{\#} \circ \varphi \circ f\right]\right)\right)_{i \in I}$.

Agora, para todo $x \in F_{n}$ e $i \in I$, tem-se:

$\left(\pi_{i} \circ \psi_{i} \circ p_{i} \circ \phi \circ \varepsilon^{\#} \circ \varphi \circ f\right)(x)=\left(\pi_{i} \circ \psi_{i} \circ p_{i} \circ \phi \circ \varepsilon^{\#} \circ \varphi\right)(f(x))=\left(\pi_{i} \circ \psi_{i} \circ p_{i} \circ \phi\right)\left(\varepsilon^{\#}(\varphi(f(x)))\right)=$ $\left(\pi_{i} \circ \psi_{i} \circ p_{i} \circ \phi\right)(\varphi(f(x)) \circ \varepsilon)=\left(\pi_{i} \circ \psi_{i} \circ p_{i}\right)\left(\varphi\left(f(x) \circ \varepsilon \circ j_{k}\right)_{k \in I}\right)=\pi_{i}\left(\circ \psi_{i}\left(\varphi(f(x)) \circ \varepsilon \circ j_{i}\right)\right)=$ $\left(\psi_{i}\left(\varphi(f(x)) \circ \varepsilon \circ j_{i}\right)\right)(1)=1 .\left(\varphi(f(x)) \circ \varepsilon \circ j_{i}\right)\left(1 . S_{i}\right)=\varphi(f(x))(1)=f(x)$. Com isso teremos $\left[\pi_{i} \circ \psi_{i} \circ p_{i} \circ \phi \circ \varepsilon^{\#} \circ \varphi \circ f\right]=[f]$ e assim $\operatorname{res}_{i}\left(\left[\pi_{i} \circ \psi_{i} \circ p_{i} \circ \phi \circ \varepsilon^{\#} \circ \varphi \circ f\right]\right)=[f]$. Daí, segue que

$\left.\left(\eta \circ \varepsilon_{n}^{*} \circ \varphi^{*}\right)[f]=\left(\left(r e s_{i}\right)_{i \in I} \circ\left(\pi_{i}^{*}\right)_{i \in I} \circ \psi^{*} \circ \gamma \circ \phi^{*}\right) \circ \varepsilon_{n}^{*} \circ \varphi^{*}\right)([f])=\left(\operatorname{res}_{i}\left(\left[\pi_{i} \circ \psi_{i} \circ p_{i} \circ \phi \circ\right.\right.\right.$ 
$\left.\left.\varepsilon^{\#} \circ \varphi \circ f\right]\right)_{i \in I}=([f])_{i \in I}=\operatorname{res}_{\mathcal{S}}^{G}([f])$, como afirmado.

Observação 2.3.3. A aplicação $J: H^{1}\left(G, \mathcal{S} ; \mathcal{F}_{T}(G)\right) \rightarrow H^{1}\left(G ; \mathcal{F}_{T}(G)\right)$ dada na sequência exata longa (teorema anterior) será denotada posteriormente, seguindo [17], por $\operatorname{res}_{G}^{(G, \mathcal{S})}: H^{1}\left(G, \mathcal{S} ; \mathcal{F}_{T}(G)\right) \rightarrow H^{1}\left(G ; \mathcal{F}_{T}(G)\right)$, e desempenhará papel importante no estudo da obstrução sing. É interessante observar que embora as notações res $s_{\mathcal{S}}^{G}$ (utilizada na sequência exata longa) $\operatorname{res}_{G}^{(G, \mathcal{S})}$ sejam parecidas, elas não indicam as mesmas aplicações, uma vez que res ${ }_{\mathcal{S}}^{G}: H^{1}(G ; M) \rightarrow \prod_{i \in I} H^{1}\left(S_{i} ; M\right)$.

O resultado seguinte nos mostra que o homomorfismo $\left(i_{0}\right)^{*}$ da sequência exata longa do par $(G, \mathcal{S})$ é (a menos de isomorfismo) o homomorfismo restrição

$$
\begin{aligned}
h: \operatorname{Hom}_{\mathbb{Z}_{2} G}\left(\mathbb{Z}_{2}(G / \mathcal{S}), M\right) & \rightarrow \operatorname{Hom}_{\mathbb{Z}_{2} G}(\Delta, M) \\
f & \left.\mapsto f\right|_{\Delta}
\end{aligned}
$$

Mais precisamente, temos:

Proposição 2.3.1. O homomorfismo $\Phi$ dada pela composição dos homomorfismos abaixo

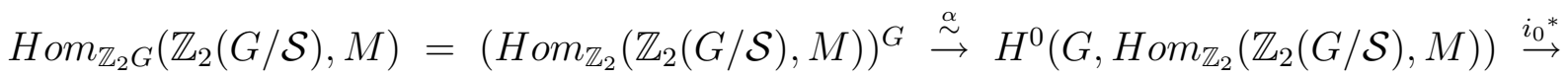
$\stackrel{i_{0}^{*}}{\rightarrow} H^{1}(G, \mathcal{S} ; M)=H^{0}\left(G ; H_{o m} \mathbb{Z}_{2}(\Delta, M)\right) \stackrel{\stackrel{\beta}{\rightarrow}}{\rightarrow}\left(H_{\mathbb{O}_{2}}(\Delta, M)\right)^{G}=H_{\mathbb{Z}_{2}}\left(\operatorname{Hom}_{\mathbb{Z}_{2} G}(\Delta, M)\right.$

é a aplicação restrição, isto é $\Phi(f)=\left.f\right|_{\Delta}$, onde $\alpha$ e $\beta$ são os isomorfismos como na demonstração da Proposição 2.1.2 e $i_{0}^{*}$ é o homomorfismo da sequência exata longa (2.5) do $\operatorname{par}(G, \mathcal{S})$.

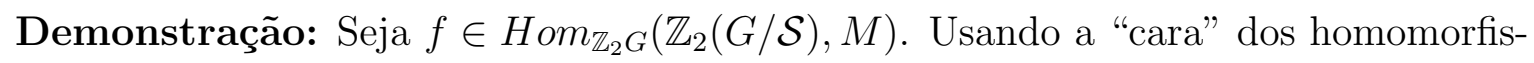
$\operatorname{mos} \alpha, \beta$ e $i_{0}^{*}$ (dados em Proposição 2.1.2; Teorema 2.3.1), e o fato que $i: \Delta \rightarrow \mathbb{Z}_{2}(G / \mathcal{S}$ ) é a inclusão, obtemos

$f \stackrel{\alpha}{\mapsto} \epsilon^{*}\left(\varphi_{f}\right)=\varphi_{f} \circ \epsilon \stackrel{i_{0}^{*}}{\mapsto} i_{0}^{*}\left(\varphi_{f} \circ \epsilon\right)=i^{\#} \circ \varphi_{f} \circ \epsilon=\epsilon^{*}\left(i_{0}^{\#} \circ \varphi_{f}\right) \stackrel{\beta}{\mapsto}\left(i^{\#} \circ \varphi_{f}\right)(1)=\varphi_{f}(1) \circ i=$ $f \circ i=\left.f\right|_{\Delta}$. 


\section{CAPÍTULO 3}

\section{DUALIDADE DE POINCARÉ}

Neste capítulo introduzimos o conceito de grupos e pares de dualidade. Um grupo de dualidade de Poincaré é um grupo cuja homologia e cohomologia satisfazem relações de dualidade análogas àquelas válidas para variedades compactas. Esses grupos foram investigados, em vista de seus aspectos algébricos e topológicos, por Bieri [7] e Johnson e Wall [14]. Posteriormente Bieri e Eckmann em [9] definiram um tipo mais geral de dualidade (sobre $\mathbb{Z}$ ) que inclui o anterior e em [8] Bieri estendeu esse conceito considerando grupos de dualidade sobre um anel $R$ qualquer, comutativo com unidade . Aqui consideraremos apenas os casos em que $R=\mathbb{Z}$ ou $\mathbb{Z}_{2}$.

\subsection{Grupos e Pares de Dualidade}

Definição 3.1.1. Um grupo $G$ é um grupo de dualidade de dimensão $n$ ou um $D^{n}$ grupo sobre $R$, se existir um $R G$-módulo $C$ tal que para todo $R G$-módulo $M$ tivermos isomorfismos

$$
H^{i}(G ; M) \simeq H_{n-i}\left(G ; C \otimes_{R} M\right)
$$

para todo $i \in \mathbb{Z}$. Nesse caso $C$ é denominado módulo dualizante.

Definição 3.1.2. A dimensão cohomológica de $G$ sobre $R$, denotada por $c d_{R} G$, é definida por

$$
\begin{aligned}
c d_{R} G & =\inf \left\{n \mid H^{k}(G ;-)=0 \text { para } k>n, n \in \mathbb{Z}\right\} \\
& =\sup \left\{n \mid H^{n}(G ; M) \neq 0 \text { para algum } R G \text {-módulo } M\right\} .
\end{aligned}
$$


Se não existir tal inteiro, nós consideramos $c d_{R} G=\infty$.

Observação 3.1.1. 1. Note que $c d_{R} G \geq 0$, pois existe $M=R$ ( $R$ visto como $R G$ módulo trivial) tal que $H^{0}(G ; R) \simeq R \neq 0$.

2. Se existe uma resolução projetiva de $R$ sobre $R G$ de comprimento finito então $c d_{R} G \leq n$.

Proposição 3.1.1. ([9], Proposição 1.2, p.106) Se G é um grupo de dualidade de dimensão $n$ sobre $R$ com módulo dualizante $C$ então:

(i) $C \neq 0$;

(ii) $C \simeq H^{n}(G ; R G)$;

(iii) $n=c d_{R} G$.

Definição 3.1.3. Se $G$ for um $D^{n}$-grupo com módulo dualizante isomorfo $C$ a $R$ então $G$ é chamado grupo de dualidade de Poincaré de dimensão n ou um $P D^{n}$-grupo (sobre $R$ ). Quando $G \simeq \mathbb{Z}$ sabemos que há duas ações possíveis de $G$ sobre $C$ a trivial $e$ a dada por g.x $=-x$. Se a ação é trivial dizemos que $G$ é um P $D^{n}$-grupo orientável, caso contrário o $P D^{n}$-grupo é dito não orientável.

Observação 3.1.2. 1. Se $C \simeq \mathbb{Z}_{2}$, a única $G$-ação sobre $C$ é a trivial e assim, sobre $\mathbb{Z}_{2}, G$ é um $P D^{n}$-grupo orientável.

2. G é um $P D^{n}$-grupo sobre $\mathbb{Z}_{2}$ se $H^{i}(G ; M) \simeq H_{n-i}(G ; M)$, para todo inteiro $i$, e todo $\mathbb{Z}_{2} G$-módulo $M$.

3. Se $G$ é grupo de dualidade sobre $\mathbb{Z}$ de dimensão $n$ com módulo dualizante $C$ então $G$ é um grupo de dualidade de dimensão n sobre $\mathbb{Z}_{2}$ com módulo dualizante $C \otimes_{\mathbb{Z}} \mathbb{Z}_{2}$.

O resultado seguinte nos dá exemplos de grupos de dualidade sobre $\mathbb{Z}$ e consequentemente sobre $\mathbb{Z}_{2}$.

Teorema 3.1.1. ([13], Teorema V.1.1, p.135) Seja G um grupo. Se existe uma variedade asférica $X$ fechada (compacta e sem bordo) de dimensão $n$ com $\pi_{1}(X)=G$ então $G$ é um grupo de dualidade de Poincaré de dimensão n sobre $\mathbb{Z}$ e consequentemente sobre $\mathbb{Z}_{2}$. 
Exemplo 3.1.1. $G=\pi_{1}\left(T^{n}\right) \simeq \mathbb{Z}^{n}$, onde $T^{n}=S^{1} \times S^{1} \times \ldots \times S^{1}$ é o toro $n$ dimensional, $n \geq 1$, são exemplos de $P D^{n}$-grupos sobre $\mathbb{Z}$ e $\mathbb{Z}_{2}$.

Definição 3.1.4. Um par grupo $(G, \mathcal{S})$ é chamado par de dualidade de dimensão $n$ ou $D^{n}$-par (sobre $R=\mathbb{Z}$ ou $\mathbb{Z}_{2}$ ) se existirem um $R G$-módulo $C$ e isomorfismos,

$$
\begin{aligned}
& H^{i}(G ; M) \simeq H_{n-i}\left(G, \mathcal{S} ; C \otimes_{R} M\right) \quad e \\
& H^{i}(G, \mathcal{S} ; M) \simeq H_{n-i}\left(G ; C \otimes_{R} M\right),
\end{aligned}
$$

para todo inteiro $i \geq 1$, e todo $R G$-módulo $M$.

Definição 3.1.5. Se $C \simeq R$, como grupo abeliano, o par grupo $(G, \mathcal{S})$ é chamado par de dualidade de Poincaré de dimensão $n$ ou $P D^{n}$-par.

Observação 3.1.3. 1. Como para grupos de dualidade, se $C \simeq \mathbb{Z}$ diz-se quem um $P D^{n}$-par é orientável (sobre $\mathbb{Z}$ ), se a $G$-ação sobre $C \simeq \mathbb{Z}$ é trivial, e é $P D^{n}$-par não orientável, caso contrário.

2. Se $C \simeq \mathbb{Z}_{2}$, $(G, \mathcal{S})$ é um $P D^{n}$-par sobre $\mathbb{Z}_{2}$ (orientável) se existir isomorfismos naturais, $H^{i}(G ; M) \simeq H_{n-i}(G, \mathcal{S} ; M)$ e $H^{i}(G, \mathcal{S} ; M) \simeq H_{n-i}(G ; M)$.

Exemplo 3.1.2. Considere $G=\langle t\rangle \bigoplus\langle s\rangle \simeq \mathbb{Z} \bigoplus \mathbb{Z}$ e $S=\left\langle t^{2}\right\rangle \bigoplus\langle s\rangle \simeq 2 \mathbb{Z} \bigoplus \mathbb{Z}$. Então $(G,\{S\})$ é um $P D^{3}$-par sobre $\mathbb{Z}_{2}$. De fato, temos que $G / S=\{\overline{(1,1)}, \overline{(t, 1)}\} \stackrel{\text { not. }}{=}\{\overline{1}, \bar{T}\} \simeq$ $\mathbb{Z}_{2}$. Considerando a aplicação aumentação, $\varepsilon: \mathbb{Z}_{2}(G / S) \rightarrow \mathbb{Z}_{2}$, temos que $\mathbb{Z}_{2}(G / S) \simeq$ $\mathbb{Z}_{2}(\{\overline{1}, \bar{T}\})=\left\{x \overline{1}+y \bar{T} ; x, y \in \mathbb{Z}_{2}\right\}=\{\overline{0}, \overline{1}, \bar{T}, \overline{1}+\bar{T}\}$. Por outro lado, $\varepsilon(\overline{0})=\overline{0}, \varepsilon(\overline{1})=$ $\overline{1}, \varepsilon(\bar{T})=\overline{1}$ e $\varepsilon(\overline{1}+\bar{T})=\varepsilon(\overline{1})+\varepsilon(\bar{T})=\overline{1}+\overline{1}=\overline{0}$, de modo que $\Delta=\operatorname{ker}(\varepsilon)=\{\overline{0}, \overline{1}+\bar{T}\} \simeq$ $\mathbb{Z}_{2}$. Com isso temos que $\operatorname{Hom}_{\mathbb{Z}_{2}}(\Delta, M) \simeq M$ e $\Delta \otimes_{\mathbb{Z}_{2}} M \simeq M$. Como Gé PD $D^{2}$-grupo, visto que $G \simeq \pi_{1}\left(T^{2}\right)$, obtemos $H_{3-k}(G, \mathcal{S}, M):=H_{3-k-1}\left(G ; \Delta \otimes_{\mathbb{Z}_{2}} M\right)=H_{2-k}(G ; M) \simeq$ $H^{k}(G ; M)$ e $H^{k}(G, \mathcal{S}, M):=H^{k-1}\left(G ; H_{o m} \mathbb{Z}_{2}(\Delta, M)\right)=H^{k-1}(G ; M) \simeq H_{3-k}(G ; M)$, donde segue que $(G, \mathcal{S})$ é um $P D^{3}-$ par.

Definição 3.1.6. Um par grupo $(G, \mathcal{S}), \mathcal{S}=\left(S_{i}\right)_{i \in I}$, é realizado topologicamente por um par Eilenberg-MacLane $(X, Y) \stackrel{\text { not. }}{=} K(G, \mathcal{S}, 1)$, se $X$ é um $K(G, 1)$ complexo celular (isto é, um complexo celular, com $X$ conexo, $\pi_{1}(X)=G$ e $\pi_{j}(X)=0, \forall j \geq 2$ ) e $Y$ é um subcomplexo cujas componentes conexas $Y_{i}, i \in I$, são $K\left(S_{i}, 1\right)$ complexos, de tal modo que as aplicações $i^{*}: \pi_{1}\left(Y_{i}\right) \rightarrow \pi_{1}(X)$, induzidas da inclusão $i: Y_{i} \rightarrow X$, são injetivas e levam cada $\pi_{1}\left(Y_{i}\right)$ em $S_{i}$, depois de uma conveniente escolha de caminhos conectados por pontos bases. 
Teorema 3.1.2. ([10], Teorema 6.3$) S e(G, \mathcal{S}), \mathcal{S}=\left(S_{i}\right), i=1, \ldots, r$, é realizado topologicamente por um par Eleinberg-MacLane $(X, Y)$, onde $X$ é uma variedade orientável, compacta, de dimensão n e $Y=\partial X\left(Y=\bigcup_{i=1}^{r} Y_{i}, Y_{i}\right.$ componentes conexas e $\left.Y_{i}=K\left(S_{i}, 1\right)\right)$ então $(G, \mathcal{S})$ é um $P D^{n}$-par orientável sobre $\mathbb{Z}$ (e portanto sobre $\mathbb{Z}_{2}$ ).

Exemplo 3.1.3. Seja $X=T^{2}-D$ (o toro menos um disco aberto), $Y=\partial X, G=$ $\pi_{1}(X) \simeq\langle a\rangle *\langle b\rangle \simeq \mathbb{Z} * \mathbb{Z}$ e $\mathcal{S}=\{S\}$, onde $S=\pi_{1}(Y) \simeq\left\langle a b a^{-1} b^{-1}\right\rangle \simeq \mathbb{Z}$. Temos que $(X, Y)$ é um par de Eleinberg-MacLane realizando $(G, \mathcal{S})$ e, como $X$ é uma variedade de dimensão 2, com bordo, compacta, orientável e $Y=\partial X$, segue que $(G, \mathcal{S})$ é um $P D^{2}$-par sobre $R=\mathbb{Z}$ ou $\mathbb{Z}_{2}$.

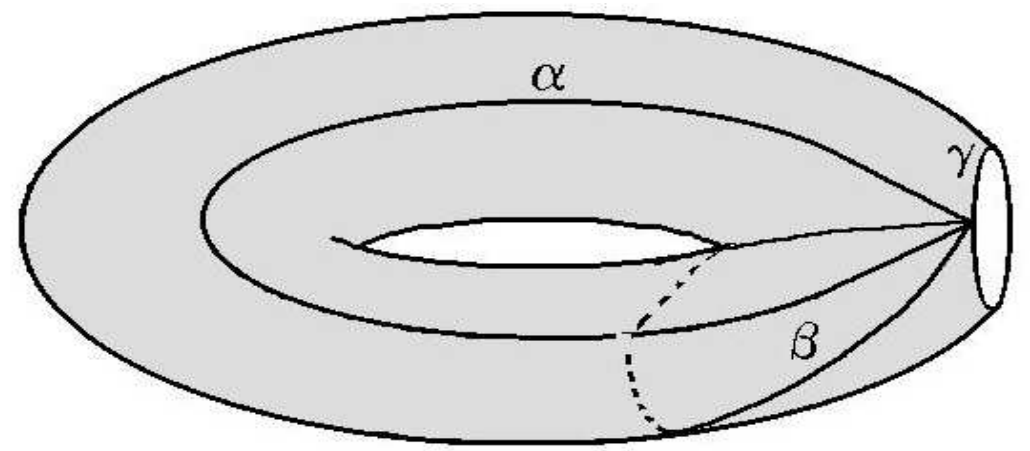

Observação 3.1.4. Se $(G, \mathcal{S})$ é um $D^{n}$-par então a família $\mathcal{S}$ é finita como mostra o resultado seguinte.

Teorema 3.1.3. ([10], Teorema 4.2) Seja $(G, \mathcal{S}), \mathcal{S}=\left(S_{i}\right)_{i \in I} \neq \emptyset$, um $D^{n}$-par com módulo dualizante C. Então

(i) G é um $D^{n-1}$-grupo com módulo dualizante $\Delta \otimes_{\mathbb{Z}_{2}} C$ (com a $G$-ação diagonal).

(ii) $\mathcal{S}$ é uma familia finita de subgrupos.

(iii) Cada $S_{i}$ é um $D^{n-1}$-grupo com módulo dualizante $C$ (considerado como $\mathbb{Z}_{2} S_{i}$-módulo por restrição).

Observação 3.1.5. Note que (i) é facilmente obtido para todo $\mathbb{Z}_{2} G$-módulo $M$, pois: $(G, \mathcal{S}) D^{n}$-par com módulo dualizante $C \Rightarrow H^{i}(G ; M) \simeq H_{n-i}\left(G, \mathcal{S} ; C \otimes_{\mathbb{Z}_{2}} M\right) \stackrel{\text { def. }}{=}$ $H_{n-1-i}\left(G ; \Delta \otimes_{\mathbb{Z}_{2}}\left(C \otimes_{\mathbb{Z}_{2}} M\right)\right)=H_{n-1-i}\left(G ;\left(\Delta \otimes_{\mathbb{Z}_{2}} C\right) \otimes_{\mathbb{Z}_{2}} M\right) \Rightarrow G$ é um $D^{n-1}$-grupo com módulo dualizante $\Delta \otimes_{\mathbb{Z}_{2}} C$. 
Corolário 3.1.1. Se $(G, \mathcal{S})$ é um $P D^{n}$-par então cada $S_{i} \in \mathcal{S}$ é um $P D^{n-1}$-grupo. 


\section{CAPÍTULO 4}

\section{$\mathrm{O} \mathbb{Z}_{2} G$-MÓDULO $\mathcal{F}_{T}(G)$ E PARES DE DUALIDADE DE POINCARÉ}

Como vimos no Exemplo 1.2.1, dado um grupo $G$, podemos dar a $\mathcal{P}(G)$ uma estrutura de $\mathbb{Z}_{2} G$-módulo. Denotemos por $\mathcal{F}(G)$ o $\mathbb{Z}_{2} G$-submódulo de $\mathcal{P}(G)$ formando por todos os subconjuntos finitos de $G$, ou seja, $\mathcal{F}(G)=\{A \in \mathcal{P}(G) ; A$ é finito $\}$. Neste capítulo apresentamos algumas propriedades do $\mathbb{Z}_{2} G$-módulo $\mathcal{P}(G)$ e do $\mathbb{Z}_{2} G$-submódulo $\mathcal{F}_{T}(G):=$ $\{A \in \mathcal{P}(G) ; A \subseteq F . T$, para algum $F \in \mathcal{F}(G)\}$ de $\mathcal{P}(G)$, onde $T$ um subgrupo de $G$. Dentre elas, destacaremos que $\mathcal{F}_{T}(G) \simeq \operatorname{Ind}_{T}^{G}\left(\mathcal{P}(T)\right.$ ), e que $\mathcal{F}_{T}(G)=\mathcal{F}_{S}(G)$, sendo $S$ um outro subgrupo de $G$ se, e somente se $S$ e $T$ são comensuráveis. Finalizamos o capítulo com uma importante caracterização dos $P D^{n}$-pares $(G, \mathcal{S})$, cuja demonstração usa o $\mathbb{Z}_{2} G$-módulo $\mathcal{F}_{T}(G)$. As referências principais para os resultados apresentados aqui são [16] e [17]. Sendo que os resultados para pares $(G, \mathcal{S})$ estão em [17]. Observamos que em [16] e [17], os autores trabalham com $\mathbb{Z}_{2} G$-módulos à direita, no entanto, em nosso tratamento, consideramos $\mathbb{Z}_{2} G$-módulos à esquerda, uma vez que as referências utilizadas para cohomologia de grupos ([11] e [10]) e para grupos e pares de dualidade ([8] e [10]) consideram $\mathbb{Z}_{2} G$-módulos à esquerda. Conforme já observamos, todo $\mathbb{Z}_{2} G$ módulo à esquerda é um $\mathbb{Z}_{2} G$-módulo à direita (e reciprocamente), e portanto é indiferente trabalharmos com $\mathbb{Z}_{2} G$-módulos à esquerda ou à direita (desde que sejamos coerente).

\section{1 $\mathcal{F}_{T}(G)$ e Comensurabilidade}

Iniciamos essa seção mostrando que $\mathcal{P}(G) \simeq \operatorname{Coind}_{\{1\}}^{G} \mathbb{Z}_{2}$ (o $\mathbb{Z}_{2} G$-módulo coinduzido, ver Capítulo $2 \S 2.1 .2$ ). 
Proposição 4.1.1. Sejam $G$ um grupo. Então $\overline{\mathbb{Z}_{2} G}:=\operatorname{Coind}_{\{1\}}^{G} \mathbb{Z}_{2}=H_{o m} \mathbb{Z}_{2}\left(\mathbb{Z}_{2} G, \mathbb{Z}_{2}\right) \simeq$ $\mathcal{P}(G)$ como $\mathbb{Z}_{2} G$-módulos. Além disso, o $\mathbb{Z}_{2} G$-submódulo $\mathbb{Z}_{2} G$ de $\overline{\mathbb{Z}_{2} G}$ é levado por este isomorfismo em $\mathcal{F}(G)$.

\section{Demonstração: Seja}

$$
\begin{aligned}
\rho: \overline{\mathbb{Z}_{2} G}=\operatorname{Coind}_{\{1\}}^{G} \mathbb{Z}_{2} & \rightarrow \mathcal{P}(G) \\
f & \mapsto \rho(f):=A_{f} ; A_{f}=\left\{g \in G ; f\left(g^{-1}\right)=1 \in \mathbb{Z}_{2}\right\}
\end{aligned}
$$

\section{Temos que:}

1. $\rho$ está bem definida, pois:

$\forall f, h \in \overline{\mathbb{Z}_{2} G}, f=h \Rightarrow f\left(g^{-1}\right)=h\left(g^{-1}\right), \forall g \in \mathbb{Z}_{2} G$. Assim, para todo $g \in \mathbb{Z}_{2} G$, $f\left(g^{-1}\right)=1 \Leftrightarrow h\left(g^{-1}\right)=1$. Logo, $A_{f}=A_{h}$.

2. $\rho$ é um homomorfismo de grupos.

De fato, mostremos que $\rho(f+h)=\rho(f)+\rho(h)$ e $\rho\left(g^{\prime} \cdot f\right)=g^{\prime} . \rho(f)$.

- $g \in \rho(f+h) \Leftrightarrow g \in A_{f+h} \Leftrightarrow(f+h)\left(g^{-1}\right)=1 \in \mathbb{Z}_{2} \Leftrightarrow f\left(g^{-1}\right)+h\left(g^{-1}\right)=1 \Leftrightarrow$ $f\left(g^{-1}\right)=1$ e $h\left(g^{-1}\right)=0$ ou $f\left(g^{-1}\right)=0$ e $h\left(g^{-1}\right)=1 \Leftrightarrow g \in A_{f} \cap\left(A_{h}\right)^{c}$ ou $g \in\left(A_{f}\right)^{c} \cap A_{h} \Leftrightarrow g \in A_{f}+A_{h} \Leftrightarrow g \in \rho(f)+\rho(h)$.

- $g \in g^{\prime} . \rho(f) \Rightarrow g \in g^{\prime} \cdot A_{f} \Rightarrow g=g^{\prime} . a$, com $a \in A_{f} \Rightarrow g \cdot a^{-1}=g^{\prime} \Rightarrow a^{-1}=$ $g^{-1} \cdot g^{\prime} \Rightarrow f\left(a^{-1}\right)=f\left(g^{-1} \cdot g^{\prime}\right) \stackrel{a \in A_{f}}{\Rightarrow} 1=f\left(g^{-1} \cdot g^{\prime}\right) \stackrel{f \in \overline{\mathbb{Z}}_{2} G}{\Rightarrow}\left(g^{\prime} \cdot f\right)\left(g^{-1}\right)=1 \Rightarrow g \in$ $A_{g^{\prime} . f} \Rightarrow g \in \rho\left(g^{\prime} . f\right)$. Agora $g \in \rho\left(g^{\prime} . f\right) \Rightarrow g \in A_{g^{\prime} . f} \Rightarrow\left(g^{\prime} . f\right)\left(g^{-1}\right)=1 \Rightarrow$ $f\left(g^{-1} \cdot g^{\prime}\right)=1 \Rightarrow g^{\prime-1} . g=a \in A_{f} \Rightarrow g=g^{\prime} a$, com $a \in A_{f} \Rightarrow g \in g^{\prime} \cdot A_{f} \Rightarrow g \in$ $g^{\prime} \cdot \rho(f)$.

3. Considere

$$
\begin{aligned}
\psi: \mathcal{P}(G) & \rightarrow \operatorname{Hom}_{\mathbb{Z}_{2}}\left(\mathbb{Z}_{2} G, \mathbb{Z}_{2}\right) \\
A & \mapsto \psi(A):=f_{A} ; f_{A}(g)= \begin{cases}1, & \text { se } g^{-1} \in A, \\
0, & \text { se } g^{-1} \notin A .\end{cases}
\end{aligned}
$$

Temos que $\psi$ está bem definida e é uma inversa para $\rho$, pois

- $\forall f \in \overline{\mathbb{Z}_{2} G},(\psi \circ \rho)(f)=\psi(\rho(f))=\psi\left(A_{f}\right)=f_{A_{f}}$. Sendo que, para todo $g \in \mathbb{Z}_{2} G, f_{A_{f}}(g)=\left\{\begin{array}{ll}1, & \text { se } g^{-1} \in A_{f}, \\ 0, & \text { se } g^{-1} \notin A_{f} .\end{array} \Leftrightarrow f_{A_{f}}(g)=\left\{\begin{array}{ll}1, & \text { se } f(g)=1 \\ 0, & \text { se } f(g)=0\end{array} \Leftrightarrow\right.\right.$ $f_{A_{f}}(g)=f(g)$.

Logo, $(\psi \circ \rho)(f)=f$. 
- $\forall A \in \mathcal{P}(G),(\rho \circ \psi)(A)=\rho(\psi(A))=\rho\left(f_{A}\right)=A_{f_{A}}=\left\{g \in G ; f_{A}\left(g^{-1}\right)=1\right\}=$ $\left\{g^{-1} \in G ; f_{A}(g)=1\right\}=A\left(\right.$ pois $\left.f_{A}(g)=1 \Leftrightarrow g^{-1} \in A\right)$.

Assim, $\rho$ é um $\mathbb{Z}_{2} G$-isomorfismo e portanto $\overline{\mathbb{Z}_{2} G} \simeq \mathcal{P}(G)$.

Agora, definamos

$$
\begin{aligned}
\varphi: \mathbb{Z}_{2} G & \rightarrow \overline{\mathbb{Z}_{2} G} \\
g^{\prime} & \mapsto \varphi\left(g^{\prime}\right) ; \varphi\left(g^{\prime}\right)(g)=\left\{\begin{array}{lc}
1, & \text { se } g=g^{\prime-1}, \\
0, & \text { caso contrário. }
\end{array}\right.
\end{aligned}
$$

e teremos que:

(i) $\varphi$ é aplicação de $\mathbb{Z}_{2} G$-módulos, pois

$$
\begin{aligned}
& \varphi\left(g_{0} \cdot g^{\prime}\right)(g)=\left\{\begin{array}{lc}
1, & \text { se } g=\left(g_{0} g^{\prime}\right)^{-1}=g^{-1} g_{0}{ }^{-1}, \\
0, & \text { caso contrário. }
\end{array}\right. \\
& \text { e } \\
& \left(g_{0} \cdot \varphi\left(g^{\prime}\right)\right)(g)=\left\{\begin{array}{lc}
1, & \text { se } g \cdot g_{0}=g^{\prime-1} \Leftrightarrow g=g^{-1} g_{0}{ }^{-1}, \\
0, & \text { caso contrário. }
\end{array}\right.
\end{aligned}
$$

Logo, $\varphi\left(g_{0} g^{\prime}\right)=g_{0} \cdot \varphi\left(g^{\prime}\right)$.

(ii) $\varphi$ é um $\mathbb{Z}_{2} G$-monomorfismo.

De fato, sejam $u=1 . g_{1}+\cdots+1 . g_{t}, u^{\prime}=1 . h_{1}+\cdots+1 . h_{r} \in \mathbb{Z}_{2} G$ tais que $u \neq u^{\prime}$.

Então existe pelo menos um índice i tal que $g_{i} \neq h_{j}$ para todo $\mathrm{j}$ (ou $h_{i} \neq g_{j}, \forall j$ ).

Daí,

$$
\begin{aligned}
& \varphi(u)\left(g_{i}^{-1}\right)=\left(1 . \varphi\left(g_{1}\right)+\cdots+1 . \varphi\left(g_{t}\right)\right)\left(g_{i}{ }^{-1}\right)=1 . \varphi\left(g_{1}\right)\left(g_{i}{ }^{-1}\right)+\cdots+1 . \varphi\left(g_{i}\right)\left(g_{i}{ }^{-1}\right)+\cdots+ \\
& 1 . \varphi\left(g_{t}\right)\left(g_{i}{ }^{-1}\right)=1 \text { e } \varphi\left(u^{\prime}\right)\left(g_{i}{ }^{-1}\right)=\left(1 . \varphi\left(h_{1}\right)+\cdots+1 . \varphi\left(h_{r}\right)\right)\left(g_{i}{ }^{-1}\right)=1 . \varphi\left(h_{1}\right)\left(g_{i}{ }^{-1}\right)+ \\
& \cdots+1 . \varphi\left(h_{r}\right)\left(g_{i}^{-1}\right)=0 .
\end{aligned}
$$

Portanto, $\varphi(u) \neq \varphi\left(u^{\prime}\right)$.

Analogamente, se $h_{i} \neq g_{j}, \forall j$, obtém-se $\varphi(u) \neq \varphi\left(u^{\prime}\right)$.

(iii) $\rho\left(\varphi\left(\mathbb{Z}_{2} G\right)\right)=\mathcal{F}(G)$.

Com efeito, seja $A \in \rho\left(\varphi\left(\mathbb{Z}_{2} G\right)\right)$. Então, $A=\rho(\varphi(x)), x \in \mathbb{Z}_{2} G$. Agora, $x \in \mathbb{Z}_{2} G$ implica que $x=1 . g_{1}+\cdots+1 . g_{t}$, com $1 \in \mathbb{Z}_{2}, g_{i} \in G$ e $g_{i} \neq g_{j}$. Daí, $\varphi(x)=$ $1 . \varphi\left(g_{1}\right)+\cdots+1 . \varphi\left(g_{t}\right)$ sendo que $\left\{\begin{array}{ll}\varphi\left(g_{i}\right)(g)=1, & \text { se } g=g_{i}{ }^{-1}, \\ \varphi\left(g_{i}\right)(g)=0, & \text { se } g \neq g_{i}{ }^{-1}\end{array}\right.$.

Logo, $A=\rho(\varphi(x))=\left\{g \in G ; \varphi(x)\left(g^{-1}\right)=1\right\}=\left\{g_{1}, \cdots, g_{t}\right\}$, ou seja, $A \in \mathcal{F}(G)$.

Reciprocamente, seja $A=\left\{g_{1}, \cdots, g_{t}\right\} \in \mathcal{F}(G)$. Podemos tomar $x=1 . g_{1}+\cdots+1 . g_{t}, x \in \mathbb{Z}_{2} G$ e daí, $\varphi(x) \in \varphi\left(\mathbb{Z}_{2} G\right)$. Como $\rho(\varphi(x))=$ $\left\{g \in G ; \varphi(x)\left(g^{-1}\right)=1\right\}=\left\{g_{1}, \cdots, g_{t}\right\}=A$, temos que $A \in \rho\left(\varphi\left(\mathbb{Z}_{2} G\right)\right)$. 
De $(i)$, (ii) segue que podemos identificar $\mathbb{Z}_{2} G \operatorname{com} \varphi\left(\mathbb{Z}_{2} G\right) \subset \overline{\mathbb{Z}_{2} G}$ e assim, $\mathbb{Z}_{2} G$ pode ser considerado um $\mathbb{Z}_{2} G$-submódulo de $\overline{\mathbb{Z}_{2} G}$. Agora de (iii) segue que esse submódulo é levado pelo isomorfismo $\rho$ em $\mathcal{F}(G)$.

Exemplo 4.1.1. (Aplicação do Lema de Shapiro) Sejam $G$ um grupo e $\mathcal{P}(G)$ o conjunto das partes de $G$. Vimos, na Proposição anterior que $\mathcal{P}(G) \simeq \operatorname{Coind}_{\{1\}}^{G} \mathbb{Z}_{2}$. Logo,

$$
H^{i}(G ; \mathcal{P}(G)) \simeq H^{i}\left(G ; \operatorname{Coind}_{\{1\}}^{G} \mathbb{Z}_{2}\right) \stackrel{\text { Shapiro }}{\simeq} H^{i}\left(\{1\} ; \mathbb{Z}_{2}\right) \simeq\left\{\begin{array}{cl}
\mathbb{Z}_{2}, & \text { se } i=0 \\
0, & \text { se } i \geq 1 .
\end{array}\right.
$$

Definição 4.1.1. ([16], p.425) Um elemento $A \in \mathcal{P}(G)$ é chamado de T-finito se existe um subconjunto finito $F$ de $G$ tal que $A \subseteq$ F.T. O conjunto de todos os subconjuntos $T$-finitos de $G$ é um $\mathbb{Z}_{2} G$-submódulo de $\mathcal{P}(G)$ denotado por $\mathcal{F}_{T}(G)$. Um subconjunto de G cujo complementar é T-finito é chamado de T-cofinito.

Observação 4.1.1. Sejam $G$ um grupo e $T$ um subgrupo de $G$

(a) Se Té finito então $\mathcal{F}_{T}(G)=\mathcal{F}(G)$.

(b) Se $T=G$ então $\mathcal{F}_{T}(G)=\mathcal{P}(G)$.

Vamos mostrar que $\mathcal{F}_{T}(G)$ é um módulo induzido.

Lema 4.1.1. Se $T$ é um subgrupo de $G$ então $\mathcal{F}_{T}(G) \simeq \operatorname{Ind}_{T}^{G} \overline{\mathbb{Z}_{2} T} \simeq \mathbb{Z}_{2} G \otimes_{\mathbb{Z}_{2} T} \mathcal{P}(T)$ como $\mathbb{Z}_{2} G$-módulo, sendo $\overline{\mathbb{Z}_{2} T}=\operatorname{Coind}_{\{1\}}^{T} \mathbb{Z}_{2}$.

Demonstração: Considere as seguintes aplicações:

$$
\operatorname{Ind}_{T}^{G} \overline{\mathbb{Z}_{2} T} \stackrel{\vartheta}{\rightarrow} \operatorname{Coind}_{T}^{G} \overline{\mathbb{Z}_{2} T} \stackrel{\nu}{\rightarrow} \overline{\mathbb{Z}_{2} G} \stackrel{\rho}{\rightarrow} \mathcal{P}(G)
$$

com $\vartheta$ e $\nu$ as $\mathbb{Z}_{2} G$-aplicações dadas, respectivamente, por:

$\vartheta\left(g^{\prime} \otimes \widetilde{f}\right)(g)=\left\{\begin{array}{ll}\left(g g^{\prime}\right) \cdot \widetilde{f}, & \text { se } g g^{\prime} \in T \\ 0, & \text { caso contrário }\end{array}, \quad \forall g^{\prime} \otimes \widetilde{f} \in \operatorname{Ind}_{T}^{G} \overline{\mathbb{Z}_{2} T}, \forall g \in G\right.$, e $\nu\left(f^{\prime}\right)(g)=f^{\prime}(g)(1), \forall g \in G, \forall f^{\prime} \in \operatorname{Coind}_{T}^{G} \overline{\mathbb{Z}_{2} T}$, sendo $1 \in \mathbb{Z}_{2} T$.

Visto que $\vartheta$ é um monomorfismo e $\nu$ é um isomorfismo ([11], Proposição III.5.9), podemos ver $\operatorname{Ind} d_{T}^{G} \overline{\mathbb{Z}_{2} T}$ como um $\mathbb{Z}_{2} G$-submódulo de $\overline{\mathbb{Z}_{2} G}$ o qual é $\mathbb{Z}_{2} G$-isomorfo (via $\rho$ ) ao $\mathbb{Z}_{2} G$-submódulo $(\rho \circ \nu \circ \vartheta)\left(\operatorname{Ind}_{T}^{G} \overline{\mathbb{Z}_{2} T}\right)$ de $\mathcal{P}(G)$. Para concluir a demonstração é suficiente verificar que $(\rho \circ \nu \circ \vartheta)\left(\operatorname{Ind}_{T}^{G} \overline{\mathbb{Z}_{2} T}\right)=\mathcal{F}_{T}(G)$. Essa igualdade segue dos seguintes fatos:

(1) $B \in \mathcal{F}_{T}(G) \Longleftrightarrow B=g_{0} T_{0} \cup \ldots \cup g_{k} T_{k}$, com $g_{i} \in G, T_{i} \subset T$ e $k \in \mathbb{N}$.

(2) Considere o $\mathbb{Z}_{2} T$-isomorfismo $\bar{\rho}: \overline{\mathbb{Z}_{2} T} \rightarrow \mathcal{P}(T)$ e a aplicação injetiva $j: \overline{\mathbb{Z}_{2} T} \rightarrow \overline{\mathbb{Z}_{2} G}$ tal que $j(\widetilde{f})=f \operatorname{com} f(g)=\left\{\begin{array}{ll}\widetilde{f}(g), & \text { se } g \in T \\ 0, & \text { caso contrário }\end{array}\right.$. Temos o diagrama comutativo: 


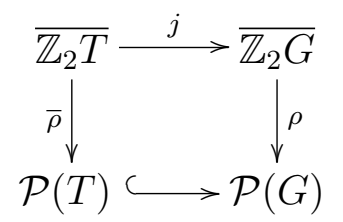

(3) Se $g_{0} \otimes \widetilde{f}_{0}$ é um gerador de $\operatorname{Ind} d_{T}^{G} \overline{\mathbb{Z}_{2} T}$, e $g \in G$,

$$
(\nu \circ \vartheta)\left(g_{0} \otimes \widetilde{f}_{0}\right)\left(g^{-1}\right)= \begin{cases}{\left[\left(g^{-1} g_{0}\right) \cdot \widetilde{f}_{0}\right](1)=\widetilde{f}_{0}\left(g^{-1} g_{0}\right),} & \text { se } g^{-1} g_{0} \in T \\ 0, & \text { caso contrário. }\end{cases}
$$

Portanto, $(\rho \circ \nu \circ \vartheta)\left(g_{0} \otimes \widetilde{f}_{0}\right)=\left\{g \in G ;(\nu \circ \vartheta)\left(g_{0} \otimes \widetilde{f}_{0}\right)\left(g^{-1}\right)=1\right\}=\left\{g \in G ; g^{-1} g_{0} \in\right.$ $T$ e $\left.\widetilde{f}_{0}\left(\left(g_{0}^{-1} g\right)^{-1}\right)=1\right\}=\left\{g \in G ; g_{0}^{-1} g \in \bar{\rho}\left(\widetilde{f}_{0}\right)\right\}=\left\{g \in G ; g_{0}^{-1} g=t \in \bar{\rho}\left(\widetilde{f}_{0}\right)\right\}=$ $\left\{g_{0} t ; t \in \bar{\rho}\left(\widetilde{f}_{0}\right)\right\}$, ou seja, $\left.(\rho \circ \nu \circ \vartheta)\left(g_{0} \otimes \widetilde{f}_{0}\right)\right)=g_{0} T_{0} \in \mathcal{F}_{T} G \operatorname{com} T_{0}=\bar{\rho}\left(\widetilde{f}_{0}\right) \subset T$. Mais geralmente, se $x=g_{0} \otimes \widetilde{f}_{0}+g_{1} \otimes \widetilde{f}_{1}+\ldots+g_{k} \otimes \widetilde{f}_{k} \in \operatorname{Ind}_{T}^{G} \overline{\mathbb{Z}_{2} T}$ então $(\rho \circ \nu \circ \vartheta)(x)=$ $g_{0} T_{0} \cup \ldots \cup g_{k} T_{k} \in \mathcal{F}_{T}(G) \operatorname{com} T_{i}=\bar{\rho}\left(\widetilde{f}_{i}\right) \subset T$ para $i=0, \ldots, k$.

(4) Dado $B=g_{0} T_{0} \cup \ldots \cup g_{k} T_{k} \in \mathcal{F}_{T}(G), B=(\rho \circ \nu \circ \vartheta)\left(g_{0} \otimes(\bar{\rho})^{-1}\left(T_{0}\right)+\ldots+g_{k} \otimes\right.$ $\left.(\bar{\rho})^{-1}\left(T_{k}\right)\right) \in(\rho \circ \nu \circ \vartheta)\left(\operatorname{Ind}_{T}^{G} \overline{\mathbb{Z}_{2} T}\right)$.

Observação 4.1.2. (i) Para um subconjunto $B$ de $G$, seja $[B]=\{H \subset G ; B+H \in$ $\left.\mathcal{F}_{T}(G)\right\}$ o conjunto de todos os subconjuntos de $G$ cuja diferença simétrica com $B$ é um conjunto T-finito. O conjunto $\{[B] ; B \subseteq G\}$ pode ser identificado com $\frac{\overline{\mathbb{Z}_{2} G}}{\mathbb{Z}_{2} G \otimes_{\mathbb{Z}_{2} T} \overline{\mathbb{Z}_{2} T}} \simeq \frac{\mathcal{P}(G)}{\mathcal{F}_{T}(G)}$ e para cada $B \in \mathcal{P}(G)$, a classe $[B]=B+\mathcal{F}_{T}(G)$ é um elemento do espaço quociente. Este espaço é um $\mathbb{Z}_{2} G$-módulo, com a $G$-ação dada por $g[B]=[g B]$.

(ii) Obviamente $[\emptyset]$ é o elemento neutro de $\frac{\mathcal{P}(G)}{\mathcal{F}_{T}(G)}$ e $[G]=[\emptyset] \Leftrightarrow G \in \mathcal{F}_{T}(G) \Leftrightarrow$ $[G: T]<\infty$. Assim, quando $[G: T]=\infty$, $[\emptyset]$ e $[G]$ são dois elementos distintos do espaço quociente $\frac{\mathcal{P}(G)}{\mathcal{F}_{T}(G)}$.

Definição 4.1.2. Sejam $G$ um grupo e $T$ um subgrupo de $G$. Um subconjunto $B$ de $G$ é $T$-quase invariante se $B+g B \in \mathcal{F}_{T}(G)$ para todo $g \in G$. É usual denotar (vide [18] $e[6])$

$$
\mathcal{A}_{T}(G):=\left\{B \subset G ; B+g B \in \mathcal{F}_{T}(G), \forall g \in G\right\}
$$

Observação 4.1.3. (i) $\mathcal{F}_{T}(G) \subset \mathcal{A}_{T}(G)$. De fato, se $B \in \mathcal{F}_{T}(G)$, então existe $F \subseteq$ $G, F$ finito, tal que $B \subseteq F T$. Deste modo, supondo $F=\left\{g_{1}, \cdots, g_{k}\right\}$ temos $F T=\left\{g_{1}, \cdots, g_{k}\right\} T$ e $g B \subseteq g F T=\left\{g g_{1}, \cdots, g g_{k}\right\} T \in \mathcal{F}_{T}(G)$ assim $g B+B \in$ $\mathcal{F}_{T}(G), \forall g \in G$, e portanto $B \in \mathcal{A}_{T}(G)$. 
(ii) $\left(\frac{\mathcal{P}(G)}{\mathcal{F}_{T}(G)}\right)^{G}=\frac{\mathcal{A}_{T}(G)}{\mathcal{F}_{T}(G)}$. De fato,

$$
\begin{aligned}
\left(\frac{\mathcal{P}(G)}{\mathcal{F}_{T}(G)}\right)^{G} & =\left\{[B] \in \frac{\mathcal{P}(G)}{\mathcal{F}_{T}(G)} ; g[B]=[B], \forall g \in G\right\} \\
& =\left\{[B] \in \frac{\mathcal{P}(G)}{\mathcal{F}_{T}(G)} ;[g B]=[B], \forall g \in G\right\} \\
& =\left\{[B] \in \frac{\mathcal{P}(G)}{\mathcal{F}_{T}(G)} ; g B+\mathcal{F}_{T}(G)=B+\mathcal{F}_{T}(G), \forall g \in G\right\} \\
& =\left\{[B] \in \frac{\mathcal{P}(G)}{\mathcal{F}_{T}(G)} ; g B+B \in \mathcal{F}_{T}(G), \forall g \in G\right\}=\frac{\mathcal{A}_{T}(G)}{\mathcal{F}_{T}(G)}
\end{aligned}
$$

Definição 4.1.3. Dizemos que dois subgrupos $S$ e $T$ de $G$ são comensuráveis se $[S: S \cap T]<\infty e[T: T \cap S]<\infty$.

Exemplo 4.1.2. (i) Obviamente, todo subgrupo é comensurável a ele mesmo.

(ii) Se $S$ e $T$ são subgrupos de $G$ com $T \subset S$ e $[S: T]<\infty$ então $S$ e $T$ são comensuráveis.

Observação 4.1.4. 1. O conjunto de todos os $g \in G$ tal que $T$ é comensurável a $T^{g}=g T g^{-1}$ será denotado por $\operatorname{Com}_{G}(T)$.

2. Usaremos a notação $S \sim T$ para dizer que um conjugado $S^{g}=g S g^{-1}$ de $S$ é comensurável a $T$, ou seja, $\left[S^{g}: S^{g} \cap T\right]<\infty e\left[T: S^{g} \cap T\right]<\infty$, para algum $g \in G$. Assim $S \nsim T$ se, para todo $g \in G, T$ e $S^{g}$ não são comensuráveis, isto é, $\left[S^{g}: S^{g} \cap T\right]=\infty$ ou $\left[T: S^{g} \cap T\right]=\infty$

Um fato interessante é que o módulo $\mathbb{Z}_{2} G \otimes_{\mathbb{Z}_{2} T} \overline{\mathbb{Z}_{2} T} \simeq \mathcal{F}_{T}(G)$ permanece inalterado se $T$ é substituído por qualquer subgrupo comensurável com $T$, conforme veremos na Proposição 4.1.3.

Proposição 4.1.2. Se T é um subgrupo de $S \operatorname{com}[S: T]<\infty$ então $\mathcal{F}_{T}(G)=\mathcal{F}_{S}(G)$.

Demonstração: Temos que $\mathcal{F}_{T}(G)=\{X \subset G ; X \subseteq F . T$, com $F$ finito $\}=$ $\left\{X \subset G ; X \subseteq g_{1} T \cup \ldots \cup g_{m} T\right\}$ (para certos elementos $g_{1}, \ldots, g_{m}$ de $G$ ). Mas obviamente $X \subseteq g_{1} T \cup \ldots \cup g_{m} T \subset g_{1} S \cup \ldots \cup g_{m} S$, se $T \subset S$. Portanto, $\mathcal{F}_{T}(G) \subset \mathcal{F}_{S}(G)$.

Para provar a outra inclusão, suponhamos que $[S: T]=k$, com isso teremos $S=$ $s_{1} T \cup \ldots \cup s_{k} T$, com $\left\{s_{1} T, \ldots, s_{k} T\right\}$ classes laterais distintas. Deste modo $X \in \mathcal{F}_{S}(G) \Rightarrow$ $X \subset F . S=F .\left(s_{1} T \cup \ldots \cup s_{k} T\right)=F . s_{1} T \cup \ldots \cup F . s_{k} T=\left(F . s_{1} \cup \ldots \cup F . s_{k}\right) . T=\widetilde{F} . T$, onde 
$\widetilde{F}=F . s_{1} \cup \ldots \cup F . s_{k}$ é finito, pois é a união finita de conjuntos finitos. Logo, $X \in \mathcal{F}_{T}(G)$ e com isso podemos concluir que $\mathcal{F}_{T}(G)=\mathcal{F}_{S}(G)$.

Proposição 4.1.3. Sejam $S$ e $T$ dois subgrupos comensuráveis de $G$. Então $S$ e $T$ são comensuráveis se, e somente se $\mathcal{F}_{S}(G)=\mathcal{F}_{T}(G)$.

Demonstração: $(\Rightarrow)$ Como $S$ e $T$ são comensuráveis temos que $[S: S \cap T]<\infty$ e $[T: S \cap T]<\infty$. Pela proposição anterior segue que $\mathcal{F}_{S}(G)=\mathcal{F}_{S \cap T}(G)=\mathcal{F}_{T}(G)$.

$(\Leftarrow)$ Suponhamos $\mathcal{F}_{S}(G)=\mathcal{F}_{T}(G)$. Se $S$ não é comensurável com $T$ então $[S: T \cap S]=\infty$ ou $[T: T \cap S]=\infty$. Suponhamos, por exemplo, que $[S: T \cap S]=\infty$ e seja $\left\{s_{\alpha} ; \alpha \in J\right\}$, com $J$ infinito, um conjunto de representantes para as classes laterais à esquerda de $S \cap T$ em $S$. Assim $S=\bigcup_{\alpha \in J} s_{\alpha}(S \cap T)$. Seja $S_{1}=\left\{s_{\alpha} ; \alpha \in I\right\} \subset S$. Obviamente $S_{1}$ é $S$-finito em $G$, isto é, $S_{1} \in \mathcal{F}_{S}(G)$, pois $S_{1} \subset S \subset\{1\} S$. Afirmamos, entretanto, que $S_{1} \notin \mathcal{F}_{T}(G)$ e portanto $\mathcal{F}_{S}(G) \neq \mathcal{F}_{T}(G)$, o que contradiz a hipótese. De fato, se $S_{1} \subset g_{1} T \cup \ldots \cup g_{k} T, g_{i} \in G$, como $S_{1}$ é finito, existem $s_{\alpha}, s_{\beta} \in S_{1}$ com $s_{\alpha} \neq s_{\beta}$ e $s_{\alpha}, s_{\beta} \in g_{i} T$ (ao mesmo $g_{i} T$ ). Assim $s_{\alpha}=g_{i} t_{\alpha}$ e $s_{\beta}=g_{i} t_{\beta}$, com $t_{\alpha}, t_{\beta} \in T$. Daí $s_{\alpha} t_{\alpha}^{-1}=s_{\beta} t_{\beta}^{-1}$ e $s_{\beta} s_{\alpha}^{-1}=t_{\beta} t_{\alpha}^{-1} \in T \cap S$. Consequentemente $s_{\alpha}(T \cap S)=s_{\beta}(S \cap T)$, o que contradiz a escolha dos $s_{\alpha}$ 's.

\section{2 $\quad \mathcal{F}_{T}(G)$ e $P D^{n}$-pares $(G, \mathcal{S})$}

Nesta seção veremos quais são os tipos de $P D^{n}$-pares sobre $\mathbb{Z}_{2}$ e mais alguns resultados envolvendo o $\mathbb{Z}_{2} G$-módulo $\mathcal{F}_{T}(G)$.

Proposição 4.2.1. ([17]], Lema 2.1) Seja $(G, \mathcal{S})$ um par grupo. Se $(G, \mathcal{S})$ é um $P D^{n}$-par e Té um $P D^{n-1}$-subgrupo de $G$ então $H^{1}\left(G, \mathcal{S} ; \mathcal{F}_{T}(G)\right) \simeq \mathbb{Z}_{2}$.

Demonstração: Do fato de $G$ ser um $P D^{n}$-par segue que $H^{1}\left(G, \mathcal{S} ; \mathcal{F}_{T}(G)\right) \simeq H_{n-1}\left(G ; \mathcal{F}_{T}(G)\right)$. Por outro lado temos, pelo Lema 4.1.1, que $\mathcal{F}_{T}(G) \simeq \mathbb{Z}_{2} G \otimes_{\mathbb{Z}_{2} T} \mathcal{P}(T)=\operatorname{Ind}_{T}^{G} \mathcal{P}(T)$. Daí, $H_{n-1}\left(G ; \mathcal{F}_{T}(G)\right) \simeq H_{n-1}\left(G ; \operatorname{Ind} d_{T}^{G} \mathcal{P}(T)\right) \stackrel{\text { Shapiro }}{\simeq}$ $H_{n-1}(T ; \mathcal{P}(T)) \stackrel{T: P D^{n-1} \text {-subg. }}{\simeq} H^{0}(T ; \mathcal{P}(T)) \simeq H^{0}\left(T ; \operatorname{Coind}_{\{1\}}^{T} \mathbb{Z}_{2}\right) \stackrel{\text { Shapiro }}{\simeq} H^{0}\left(\{1\} ; \mathbb{Z}_{2}\right)=$ $\left(\mathbb{Z}_{2}\right)^{\{1\}}=\mathbb{Z}_{2}$.

Exemplo 4.2.1. Se $(G, \mathcal{S})$ é o $P D^{3}$-par do Exemplo 3.1.2 e $T$ é um $P D^{2}$-subgrupo de $G$, ou $(G, \mathcal{S})$ é o $P D^{2}$-par do Exemplo 3.1 .3 e $T$ é um $P D^{1}$-subgrupo de $G$, então $H^{1}\left(G, \mathcal{S} ; \mathcal{F}_{T}(G)\right) \simeq \mathbb{Z}_{2}$.

Vejamos agora mais detalhadamente quais são os tipos de $P D^{n}$-pares sobre $\mathbb{Z}_{2}$. 
Proposição 4.2.2. ([17], Lema 2.2) Seja $(G, \mathcal{S})$ um $P D^{n}$-par com $\mathcal{S}=\left(S_{i}\right)_{i \in I} \neq \emptyset e$ $I=\{1, \ldots, k\}$, então uma das seguintes afirmações é verdadeira.

(i) $G$ é um $P D^{n-1}$-grupo e $\mathcal{S}$ consiste de apenas um grupo $S$ com $[G: S]=2$.

(ii) $G$ é um $P D^{n-1}$-grupo e $\mathcal{S}$ consiste de duas cópias de $G$.

(iii) $[G: S]=\infty, \operatorname{Com}_{G}(S)=S$ e $S \nsim T$, para todo $S \neq T \in \mathcal{S}$.

Demonstração: Como $(G, \mathcal{S})$ é um $P D^{n}$-par, segue do Teorema 3.1.3 e do Corolário 3.1.1 que $G$ é um $D^{n-1}$-grupo com módulo dualizante $\Delta$ e todo $S \in \mathcal{S}$ é um $P D^{n-1}$-grupo. Logo, se $T \in \mathcal{S}$, da Proposição 4.2.1 tem-se que $H^{1}\left(G, \mathcal{S} ; \mathcal{F}_{T}(G)\right) \simeq \mathbb{Z}_{2}$.

Para (i) e (ii) suponhamos que exista $T \in \mathcal{S}$ tal que $[G: T]<\infty$ e considere a sequência exata longa para o par $(G, \mathcal{S})$ com coeficientes em $\mathcal{F}_{T}(G)$,

$0 \rightarrow H^{0}\left(G ; \mathcal{F}_{T}(G)\right) \rightarrow H^{0}\left(\mathcal{S} ; \mathcal{F}_{T}(G)\right) \rightarrow H^{1}\left(G, \mathcal{S} ; \mathcal{F}_{T}(G)\right) \rightarrow H^{1}\left(G ; \mathcal{F}_{T}(G)\right) \rightarrow \cdots$

Sabemos que, $\mathcal{F}_{T}(G) \simeq \mathbb{Z}_{2} G \otimes_{\mathbb{Z}_{2} T} \mathcal{P}(T)=\operatorname{Ind}_{T}^{G} \mathcal{P}(T)=\operatorname{Coind}_{T}^{G} \mathcal{P}(T)$, pois $[G: T]<\infty$. Daí

$$
\begin{aligned}
& H^{0}\left(G ; \mathcal{F}_{T}(G)\right) \simeq H^{0}\left(G ; \operatorname{Coind}_{T}^{G} \mathcal{P}(T)\right) \stackrel{\text { Shapiro }}{\simeq} H^{0}(T ; \mathcal{P}(T)) \stackrel{4.1 .1}{=} \mathbb{Z}_{2}, \\
& H^{1}\left(G ; \mathcal{F}_{T}(G)\right) \simeq H^{1}\left(G ; \operatorname{Coind}_{T}^{G} \mathcal{P}(T)\right) \stackrel{\text { Shapiro }}{\simeq} H^{1}(T ; \mathcal{P}(T)) \stackrel{4.1 .1}{=} 0 .
\end{aligned}
$$

Com isso temos a seguinte sequência exata curta:

$$
0 \longrightarrow \mathbb{Z}_{2} \longrightarrow H^{0}\left(\mathcal{S} ; \mathcal{F}_{T}(G)\right) \longrightarrow \mathbb{Z}_{2} \longrightarrow 0
$$

Como $\mathbb{Z}_{2}$ é $\mathbb{Z}_{2}$-livre, temos que (4.1) cinde e consequentemente teremos

$$
H^{0}\left(\mathcal{S} ; \mathcal{F}_{T}(G)\right) \simeq \mathbb{Z}_{2} \bigoplus \mathbb{Z}_{2}
$$

Agora $H^{0}\left(\mathcal{S}, \mathcal{F}_{T}(G)\right)=\prod_{i \in I} H^{0}\left(S_{i}, \mathcal{F}_{T}(G)\right) \stackrel{\text { Ifinito }}{\simeq} \bigoplus_{i \in I} H^{0}\left(S_{i}, \mathcal{F}_{T}(G)\right)$, e ainda $H^{0}\left(S_{i}, \mathcal{F}_{T}(G)\right)=$ $\left(\mathcal{F}_{T}(G)\right)^{S_{i}}=\left(\operatorname{Coind}_{\{1\}}^{G} \mathbb{Z}_{2}\right)^{S_{i}}=\left(\operatorname{Hom}_{\mathbb{Z}_{2}}\left(\mathbb{Z}_{2} G, \mathbb{Z}_{2}\right)\right)^{S_{i}}=H_{\text {om }} \mathbb{Z}_{2} S_{i}\left(\mathbb{Z}_{2} G, \mathbb{Z}_{2}\right)$, e $H_{o m} \mathbb{Z}_{2} S_{i}\left(\mathbb{Z}_{2} G, \mathbb{Z}_{2}\right) \simeq \operatorname{Hom}_{\mathbb{Z}_{2}}\left(\mathbb{Z}_{2}\left(G / S_{i}\right), \mathbb{Z}_{2}\right)$, com $\mathbb{Z}_{2}\left(G / S_{i}\right)$ o $\mathbb{Z}_{2}$-módulo livre gerado pelas classes $g S_{i}$, ou seja, $\mathbb{Z}_{2}\left(G / S_{i}\right) \simeq \bigoplus_{j=1}^{\left[G: S_{i}\right]}\left(\mathbb{Z}_{2}\right)_{j}$, onde $\left(\mathbb{Z}_{2}\right)_{j}=\mathbb{Z}_{2}$, para todo $j$.

Logo,

$H^{0}\left(S_{i} ; \mathcal{F}_{T}(G)\right) \simeq \operatorname{Hom}_{\mathbb{Z}_{2}}\left(\mathbb{Z}_{2}\left(G / S_{i}\right), \mathbb{Z}_{2}\right) \simeq \operatorname{Hom}_{\mathbb{Z}_{2}}\left(\bigoplus_{j=1}^{\left[G: S_{i}\right]}\left(\mathbb{Z}_{2}\right)_{j}, \mathbb{Z}_{2}\right) \simeq \prod_{j=1}^{\left[G: S_{i}\right]} \operatorname{Hom}_{\mathbb{Z}_{2}}\left(\left(\mathbb{Z}_{2}\right)_{j}, \mathbb{Z}_{2}\right) \simeq$ $\prod_{j=1}^{\left[G: S_{i}\right]}\left(\mathbb{Z}_{2}\right)_{j}$. Deste modo, $H^{0}\left(S_{i} ; \mathcal{F}_{T}(G)\right)$ contém pelo menos uma cópia de $\mathbb{Z}_{2}$, visto que
$\left[G: S_{i}\right] \geq 1$, e de $(4.2)$ concluímos que a família $\mathcal{S}$ tem no máximo dois elementos, isto é,
$k=|I|=|\mathcal{S}| \leq 2$. 

$\left[G: S_{i}\right]$

Se $k=1$, teremos $\mathcal{S}=\{S\} . \quad \operatorname{Logo}, \mathbb{Z}_{2} \bigoplus \mathbb{Z}_{2}=H^{0}\left(\mathcal{S} ; \mathcal{F}_{T}(G)\right)=H^{0}\left(S ; \mathcal{F}_{T}(G)\right) \simeq$ $\bigoplus_{j=1}\left(\mathbb{Z}_{2}\right)_{j}$ e isso implica em $[G: S]=2$ (e necessariamente $S=T$, o subgrupo inicial).

Se $k=2$, temos $\mathcal{S}=\left\{S_{1}, S_{2}\right\}$ (um deles obviamente igual a $T$ ). Daí temos $\mathbb{Z}_{2} \bigoplus \mathbb{Z}_{2} \stackrel{4.2}{\simeq}$ $H^{0}\left(\mathcal{S} ; \mathcal{F}_{T}(G)\right) \simeq \bigoplus_{i=1}^{2} H^{0}\left(S_{i} ; \mathcal{F}_{T}(G)\right)$, donde segue que, para cada $i \in\{1,2\}$, $\mathbb{Z}_{2} \simeq H^{0}\left(S_{i} ; \mathcal{F}_{T}(G)\right) \simeq \bigoplus_{j=1}^{\left[G: S_{i}\right]} H^{0}\left(S_{i} ; \mathcal{F}_{T}(G)\right) \simeq \bigoplus_{j=1}^{\left[G: S_{i}\right]} \mathbb{Z}_{2}$, e portanto $\left[G: S_{i}\right]=1$, e as$\operatorname{sim} S_{i}=G$, para cada $i \in\{1,2\}$. Com isso temos $(i)$ e $(i i)$ (Notemos que nesses dois casos $G$ é um $P D^{n-1}$-grupo, isso segue de [8], Teorema 9.9, p.157, usando que $G$ é um $D^{n-1}$-grupo (logo não tem $\mathbb{Z}_{2^{-}}$torção), $T$ é um $P D^{n-1}$-grupo e $[G: T]<\infty$ ).

Observemos que da demonstração de $(i)$ e $(i i)$ apresentada, segue que "se $\mathcal{S}$ tem subgrupo de índice finito em $G$ então $\mathcal{S}$ não terá nenhum subgrupo de índice infinito".

(iii) Suponha agora que todo $T \in \mathcal{S}$ tem índice infinito em $G$, então $H^{0}\left(G ; \mathcal{F}_{T}(G)\right)=$ $\left(\mathcal{F}_{T}(G)\right)^{G}=\left(\operatorname{Ind}_{T}^{G} \mathcal{P}(T)\right)^{G} \stackrel{\text { Prop.2.1.7 }}{=} 0$. Logo a sequência exata longa para o par $(G, \mathcal{S})$ com coeficientes em $\mathcal{F}_{T}(G)$ tem a forma

$$
0 \rightarrow H^{0}\left(\mathcal{S} ; \mathcal{F}_{T}(G)\right) \rightarrow H^{1}\left(G, \mathcal{S} ; \mathcal{F}_{T}(G)\right) \rightarrow H^{1}\left(\mathcal{S} ; \mathcal{F}_{T}(G)\right) \rightarrow \ldots
$$

de modo que podemos ver $H^{0}\left(\mathcal{S} ; \mathcal{F}_{T}(G)\right)=\bigoplus_{i \in I}\left(\mathcal{F}_{T}(G)\right)^{S_{i}}$ como um submódulo de $H^{1}\left(G, \mathcal{S} ; \mathcal{F}_{T}(G)\right) \simeq \mathbb{Z}_{2}$. Seja $S \in \mathcal{S}$.

- Afirmamos que um elemento $S g \in\left(\mathcal{F}_{T}(G)\right)^{S}$ se, e somente se $g^{-1} S g$ é $T$-finito, isto é, existe $F \subseteq G$, finito tal que $g^{-1} S g \subseteq F . T$.

Com efeito, suponhamos que $S g \in\left(\mathcal{F}_{T}(G)\right)^{S}$. Então $S g \in \mathcal{F}_{T}(G)$ e $s S g=S g$, para todo $s \in S$. Daí $g^{-1} S g \subseteq g^{-1} F_{1} . T=F_{2} . T$, com $F_{1}$ e $F_{2}=g_{-1} F_{1}$ subconjuntos finitos de $G$. Reciprocamente, se $g^{-1} S g \subseteq F_{3} T$, com $F_{3}$ finito, então $S g \subseteq g F_{3} \cdot T=F_{4} \cdot T \in$ $\mathcal{F}_{T}(G)$ e claramente $s S g=S g$, para todo $s \in S$, de modo que $S g \in\left(\mathcal{F}_{T}(G)\right)^{S}$.

- Afirmamos ainda que $H^{0}\left(S ; \mathcal{F}_{T}(G)\right)=\left(\mathcal{F}_{T}(G)\right)^{S}$ só tem elementos deste tipo, isto é, $\left(\mathcal{F}_{T}(G)\right)^{S}$ é gerado pelos elementos $S g, g \in G$ que são $T$-finitos.

De fato, dado $A \in\left(\mathcal{F}_{T}(G)\right)^{S}$ tem-se $A \subseteq F . T$, com $F$ finito e $s A=A$, para todo $s \in S$. Consequentemente $S A=A$, assim $A=\bigcup_{g \in A} S g$.

Sejam $g_{j} \in A, j \in J$, elementos tais que $S g_{i} \neq S g_{j}$ se $g_{i} \neq g_{j}$ (representantes para as classes laterais). Então $A=\bigcup_{j \in J} S g_{j}$ e a soma (dado pela diferença simétrica) $S g_{i}+S g_{j}=\left(S g_{i} \cup S g_{j}\right)-\left(S g_{i} \cap S g_{j}\right)=S g_{i} \cup S g_{j}$.

- Agora, claramente $S g \in\left(\mathcal{F}_{T}(G)\right)^{S}$ para $S=T$ e $g \in T$, ou seja, $T \in\left(\mathcal{F}_{T}(G)\right)^{T}=$ $\{\emptyset, T\} \simeq \mathbb{Z}_{2}$ e $\left(\mathcal{F}_{T}(G)\right)^{S}=\{\emptyset\}$ se $S \neq T$, pois supondo por exemplo $S_{1}=T \in \mathcal{S}$ temos 


$$
H^{0}(\mathcal{S} ; \mathcal{P}(G)) \simeq \bigoplus_{i \in I} H^{0}\left(S_{i} ; \mathcal{F}_{T}(G)\right)=\left(\mathcal{F}_{T}(G)\right)^{T} \bigoplus\left(\bigoplus_{i \in I, i \geq 2}\left(\mathcal{F}_{T}(G)\right)^{S_{i}}\right)
$$

que é um submódulo de $H^{1}\left(G, \mathcal{S} ; \mathcal{F}_{T}(G)\right) \simeq \mathbb{Z}_{2}$.

- Verifiquemos que $\operatorname{Com}_{G}(S)=S$, para todo $S \in \mathcal{S}$. Claramente $S \subset C \operatorname{Com}_{G}(S)$ e, se $g \in \operatorname{Com}_{G}(S)$, então $\left[S^{g}: S \cap S^{g}\right]<\infty$ e $\left[S: S \cap S^{g}\right]<\infty$, onde $S^{g}=g S g^{-1}$. Daí, $S^{g}=g S g^{-1}=u_{1}\left(S \cap S^{g}\right) \cup \ldots \cup u_{k}\left(S \cap S^{g}\right) \subseteq \bigcup_{i=1}^{k} u_{i} S=F . S, \operatorname{com} F=\left\{u_{1}, \ldots, u_{k}\right\}$ finito um conjunto de representantes das classes laterais à esquerda de $S \cap S^{g} \mathrm{em}$ $S$. Assim $g S g^{-1}$ é $S$-finito e $S g^{-1} \in\left(\mathcal{F}_{S}(G)\right)^{S}=\{\emptyset, S\}$. Daí $S g^{-1}=S$, de modo que $g \in S$ e portanto $\operatorname{Com}_{G}(S)=S$.

- Finalmente mostremos que $S \nsim T$ se $S \neq T$.

Suponhamos por absurdo que $S \sim T$ e $S \neq T$. Então existe $g \in G$ tal que $S^{g}$ é comensurável com $T$, ou seja, $\left[T: S^{g} \cap T\right]<\infty$ e $\left[S^{g}: S^{g} \cap T\right]<\infty$. De modo similar ao raciocínio anterior, obtemos $S^{g}=v_{1}\left(S^{g} \cap T\right) \cup \ldots \cup v_{r}\left(S^{g} \cap T\right)=F . T$, onde $v_{i} \in S^{g}, i=1, \ldots, r$ são representantes para as classes laterais de $S^{g} \cap T$ em $S^{g}$. Daí $S g \in\left(\mathcal{F}_{T}(G)\right)^{S}=\{\emptyset\}$, se $S \neq T$, o que é uma contradição.

Observação 4.2.1. O resultado anterior também foi demonstrado em [5] para $D^{n}$-pares usando uma outra técnica. 


\section{CAPÍTULO 5}

\section{DECOMPOSIÇÃO DE GRUPOS E}

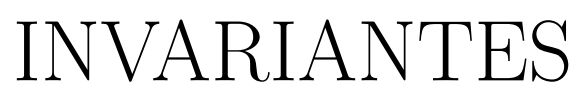

Neste capítulo apresentamos o conceito de decomposição de grupos, alguns exemplos, e resultados envolvendo os invariantes $e(G)$ e $e(G, S)$ estudados em [25] e [24].

\subsection{Decomposição de Grupos}

Definição 5.1.1. Dizemos que um grupo $G$ é definido por geradores $X=\left\{x_{i}\right\}_{i}$ e relações $R=\left\{r_{j}=1\right\}_{j}$ se $G \simeq \frac{F}{H}$ onde $F$ é um grupo livre gerado por $X$ e $H$ é o menor subgrupo normal de $F$ gerado por $\left\{r_{j}\right\}$. Neste caso dizemos que $\langle X ; R\rangle$ é uma apresentação de $G$.

Definição 5.1.2. Sejam $H$ e $K$ grupos, com apresentações $H=\left\langle X_{1} ; R_{1}\right\rangle$ e $K=$ $\left\langle X_{2} ; R_{2}\right\rangle$. Considere $S$ um subgrupo de $H$ e $T$ um subgrupo de $K$, e suponhamos que $S$ e $T$ são isomorfos via $\theta: S \stackrel{\sim}{\rightarrow} T$. Então o produto livre amalgamado de $H$ e $K$ em $S \simeq T$, ou o produto livre de $H$ e $K$ amalgamado em $S$, é definido por $G=H *_{S} K:=<X_{1}, X_{2} ; R_{1}, R_{2}, s=\theta(s), \forall s \in S>$. Se $S=\{1\}$ então $H *_{S} K$ é chamado de produto livre de $H$ e $K$ e é denotado simplesmente por $H * K$.

Exemplo 5.1.1. Consideremos $H=\langle a\rangle \simeq \mathbb{Z}$ e $K=\langle b\rangle \simeq \mathbb{Z}$. Então o produto livre de $H$ 
e K é o grupo livre com dois geradores:

$$
H * K=\langle a\rangle *\langle b\rangle=\langle a, b\rangle \simeq \mathbb{Z} * \mathbb{Z}
$$

Exemplo 5.1.2. Sejam $H=\left\langle x ; x^{2}=1\right\rangle \simeq \mathbb{Z}_{2}$ e $K=\left\langle y ; y^{2}=1\right\rangle \simeq \mathbb{Z}_{2}$. Então $G=$ $H * K=\left\langle x, y ; x^{2}=y^{2}=1\right\rangle \simeq \mathbb{Z}_{2} * \mathbb{Z}_{2}$. G é chamado grupo diedral infinito.

Exemplo 5.1.3. Sejam $H=\left\langle a_{1}\right\rangle *\left\langle b_{1}\right\rangle=\left\langle a_{1}, b_{1} ; \emptyset\right\rangle, K=\left\langle a_{2}\right\rangle *\left\langle b_{2}\right\rangle=\left\langle a_{2}, b_{2} ; \emptyset\right\rangle, S_{1}=$ $\left\langle a_{1} b_{1} a_{1}^{-1} b_{1}^{-1}\right\rangle$ e $S_{2}=\left\langle b_{2} a_{2} b_{2}^{-1} a_{2}^{-1}\right\rangle$. Então, considerando o isomorfismo $\sigma: S_{1} \rightarrow S_{2}$ definido por $\sigma\left(a_{1} b_{1} a_{1}^{-1} b_{1}^{-1}\right)=b_{2} a_{2} b_{2}^{-1} a_{2}^{-1}$, temos que

$$
\begin{aligned}
H *_{S} K & =\left\langle a_{1}, b_{1}, a_{2}, b_{2} ; a_{1} b_{1} a_{1}^{-1} b_{1}^{-1}=b_{2} a_{2} b_{2}^{-1} a_{2}^{-1}\right\rangle \\
& =\left\langle a_{1}, b_{1}, a_{2}, b_{2} ; a_{1} b_{1} a_{1}^{-1} b_{1}^{-1} a_{2} b_{2} a_{2}^{-1} b_{2}^{-1}=1\right\rangle .
\end{aligned}
$$

Definição 5.1.3. Seja $H$ um grupo com apresentação $H=\left\langle X_{1} ; R_{1}\right\rangle$. Se $S, T$ são subgrupos de $H$ com um dado isomorfismo $\theta: S \rightarrow T$ então o HNN-grupo ou HNNextensãa $H *_{S, \theta}$ sobre o grupo base $H$ (subgrupo associado a $S$ ), com respeito a $\theta$ e letra estável $p$, é definido por: $H *_{S, \theta}=\left\langle X_{1}, p ; R_{1}, p^{-1} s p=\theta(s), \forall s \in S\right\rangle$.

Exemplo 5.1.4. Se $H=\{1\}, S=T=\{1\}$ e $\theta=i d_{\{1\}}$ então $\{1\} *_{\{1\}, \theta}=H *_{S, \theta}=$ $\left\langle 1, p ; p^{-1} 1 p=1\right\rangle=\langle 1, p\rangle=\langle p\rangle \simeq \mathbb{Z}$.

Exemplo 5.1.5. Seja $H=\left\langle x, y ; x y=y x^{2}\right\rangle$. Se $H=S=\langle x\rangle$ e $T=\left\langle x^{2}\right\rangle$ então $G=S *_{S, \theta}$, com y como letra estável e o isomorfismo $\theta: S \rightarrow T$ dado por $\theta(x)=x^{2}$.

De fato, $S *_{S, \theta}=\left\langle x, y ; y^{-1} s y=\theta(s), \forall s \in S\right\rangle$

$$
=\left\langle x, y ; s y=y s^{2}, \forall s \in S\right\rangle=\left\langle x, y ; x y=y x^{2}\right\rangle=G
$$

Exemplo 5.1.6. Seja $G=\mathbb{Z} \oplus \mathbb{Z}=\langle a\rangle \oplus\langle b\rangle=\langle a, b ; a b=b a\rangle$. Sejam $G_{1}=S=S^{\prime}=$ $\langle a\rangle, \quad \theta=i d: S \rightarrow S$ e considere b como letra estável. Então $G=S *_{S, \theta}$.

De fato, $S *_{S, \theta}=\left\langle a, b ; b^{-1} s b=\theta(s), \forall s \in S\right\rangle$

$$
\begin{aligned}
& =\left\langle a, b ; b^{-1} a b=\theta(a)=a\right\rangle \\
& =\langle a, b ; a b=b a\rangle=G
\end{aligned}
$$

Em outras palavras, $\mathbb{Z} \oplus \mathbb{Z}=\mathbb{Z} *_{\mathbb{Z}, i d}$.

Observação 5.1.1. Podemos dar uma interpretação geométrica para os grupos dos Exemplos 5.1 .3 e 5.1 .6

(i) O grupo $H *_{S} K$ dado no Exemplo 5.1.3 é o grupo fundamental da soma conexa de 2 toros. Isto segue do Teorema de Van-Kampen ([11], Teorema II.7.2 ou [19], IV, 
5.3), considerando $X_{1}, X_{2}$ e $Y=X_{1} \cap X_{2}$ como na figura abaixo, e $H=\pi_{1}\left(X_{1}\right)$, $K=\pi_{1}\left(X_{2}\right), \quad S=\pi_{1}\left(\partial X_{1}\right)$ e $T=\pi_{1}\left(\partial X_{2}\right)$.

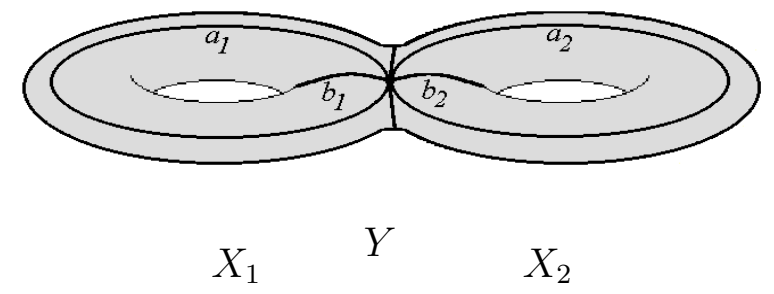

(ii) Para o Exemplo 5.1.6, considere $X$ o Toro, e $X_{1}$ e $X_{2}$ como sendo os subespaços de $X$ representados na figura pelos caminhos fechados a e b, respectivamente. Sejam $\alpha=[a]$ e $\beta=[b]$ (classes de homotopia). Temos que $S=\pi_{1}\left(X_{1}\right)=\langle\alpha>$, $\pi_{1}\left(X_{2}\right)=<\beta>$ e $\pi_{1}(X)=S *_{S, \theta}=G \simeq \mathbb{Z} \oplus \mathbb{Z}$.

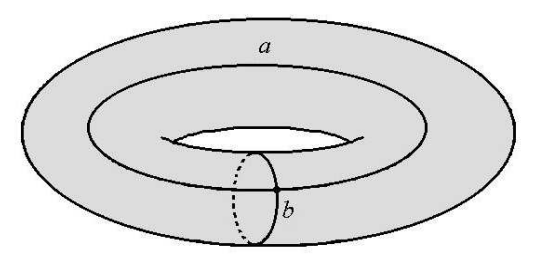

Definição 5.1.4. ( [16], p.42 ou [24], p.179) Dizemos que um grupo G se decompõe sobre um subgrupo $S$, se $G$ é:

(i) um produto livre amalgamado, não trivial, de $H$ e $K$ em $S$, isto é, $G=H *_{S} K$, com $H \neq S \neq K$, ou

(ii) uma $H N N$-extensão com subgrupo associado $S$, isto é, $G=H *_{S, \theta}$.

\subsection{Invariantes $e(G)$ e $e(G, S)$}

Decomposição de grupos sobre subgrupos finitos foi estudada por Stallings [26] e completamente caracterizada quando $G$ é finitamente gerado via o invariante end $e(G)$.

Definição 5.2.1. Sejam $G$ um grupo e $\mathcal{P}(G)$ o $\mathbb{Z}_{2} G$-módulo dado pelo conjunto das partes de $G$ com a operação adição sendo a operação diferença simétrica. Sejam $\mathcal{F}(G)$ o conjunto formado por todos os subconjuntos finitos de $G$ e $\mathcal{Q}(G):=\{A \in \mathcal{P}(G)$; para todo $g \in$ $G, \quad A+g A \in \mathcal{F}(G)\} \mathbb{Z}_{2} G$-submódulos de $\mathcal{P}(G)$. O número de ends do grupo $G$, que denotamos por $e(G)$, é definido por:

$$
e(G):=\operatorname{dim}_{\mathbb{Z}_{2}}\left(\frac{\mathcal{Q}(G)}{\mathcal{F}(G)}\right)=\operatorname{dim}_{\mathbb{Z}_{2}}\left(\frac{\mathcal{P}(G)}{\mathcal{F}(G)}\right)^{G}=\operatorname{dim}_{\mathbb{Z}_{2}} H^{0}\left(G ; \frac{\mathcal{P}(G)}{\mathcal{F}(G)}\right)
$$


Observação 5.2.1. Para maiores detalhes sobre e $(G)$ vide [25], § 5, p.171 ou [23].

Pode-se mostrar

que:

(1) $e(G)$ assume apenas os valores $0,1,2$ e $\infty$.

(2) $e(G)=0$ se, e somente se, $G$ é finito.

(3) $e(\mathbb{Z})=2$.

(4) $e(\mathbb{Z} \oplus \mathbb{Z})=1$.

(5) $e\left(\mathbb{Z}_{2} * \mathbb{Z}_{2}\right)=2$.

(6) $e(\mathbb{Z} * \mathbb{Z})=\infty$

O resultado de Stallings sobre decomposição de grupos, conhecido como Teorema de Estrutura é o seguinte:

Teorema 5.2.1. ([26], Teorema 5.A.9 ou [21], Teorema 4.1.5, p.75) Se G é um grupo finitamente gerado, então $G$ se decompõe sobre um subgrupo finito se, e somente se, $e(G) \geq 2$.

Exemplo 5.2.1. 1. $G=\mathbb{Z} * \mathbb{Z}=\mathbb{Z} *_{\{1\}} \mathbb{Z}$, e $e(\mathbb{Z} * \mathbb{Z})=\infty$.

2. $\mathbb{Z}_{2} * \mathbb{Z}_{2}=\mathbb{Z}_{2} *\{1\} \mathbb{Z}_{2}$, e e $\left(\mathbb{Z}_{2} * \mathbb{Z}_{2}\right)=2$.

3. $\mathbb{Z}=\{1\} *_{\{1\}, i d}=<\{1\}, p, p s p^{-1}=s, \forall s \in\{1\}>=<p>e \quad e(\mathbb{Z})=2$.

Observação 5.2.2. 1. A implicação : "G se decompõe sobre um subgrupo finito $\Longrightarrow$ $e(G) \geq 2$ ", é válida para $G$ não necessariamente finitamente gerado ([25], Lema $6.3)$.

2. De fato prova-se que $e(G)=2 \Longleftrightarrow G=S *_{S, \theta}$, com $S$ finito ou $G=H *_{S} K$, com $S$ finito $e[H: S]=[K: S]=2\left(\right.$ [25], Teorema 5.12). E, $e(G)=\infty \Longleftrightarrow G=H *_{S} K$ com $S$ finito contido propriamente em ambos os fatores e de indice $>2$ em ao menos um deles, ou $G=H *_{S}$, onde $S$ é finito, mergulhado propriamente em $H$ ( [26], 5.A.10).

O número de ends de um grupo infinito $G$ pode ser expressado em termos de cohomologia de grupos (no nível 1), mais precisamente, temos: 
Proposição 5.2.1. Se $G$ é um grupo infinito, então e $(G)=1+\operatorname{dim}_{\mathbb{Z}_{2}} H^{1}\left(G ; \mathbb{Z}_{2} G\right)$.

Notemos que, como $e(\mathbb{Z} \oplus \mathbb{Z})=1$, segue do resultado de Stallings que $\mathbb{Z} \oplus \mathbb{Z}$ não se decompõe sobre um subgrupo finito, entretanto $\mathbb{Z} \oplus \mathbb{Z}$ se decompõe sobre um subgrupo infinito, pois como vimos, $\mathbb{Z} \oplus \mathbb{Z}$ é o $H N N$-grupo $\mathbb{Z} * \mathbb{Z}$, id .

Observação 5.2.3. Scott em [24] (1977), tentou generalizar o resultado de Stallings para grupos que se decompõem sobre subgrupos infinitos usando um invariante para par grupo $(G, S)$.

Definição 5.2.2. Sejam $G$ um grupo e $S$ um subgrupo de $G$. Define-se o invariante end $e(G, S)$, para o par $(G, S)$, como

$$
e(G, S)=\operatorname{dim}_{\mathbb{Z}_{2}}(\mathcal{P}(G / S) / \mathcal{F}(G / S))^{G} .
$$

Usando tal invariante Scott ([24], Lema 1.8 ou [25], Lema 8.3) mostrou o resultado seguinte:

Proposição 5.2.2. Se $G$ se decompõe sobre $S$ então $e(G, S) \geq 2$.

Observação 5.2.4. 1. Pode-se verificar que $e(Z \oplus \mathbb{Z}, \mathbb{Z})=e(Z \oplus \mathbb{Z} / \mathbb{Z})=e(\mathbb{Z})=2$, e como observamos, tem-se a decomposição $\mathbb{Z} \oplus \mathbb{Z}=\mathbb{Z} *_{\mathbb{Z}, \text { id }}$.

2. A recíproca da proposição anterior não é verdadeira (vide [25], Teorema 8.4, p.200). 


\section{CAPÍTULO 6}

\section{OBSTRUÇÕES E INVARIANTES COHOMOLÓGICOS}

Como já mencionado na introdução, em [16], Kropholler e Roller, apresentaram um interessante resultado sobre decomposição de grupo de dualidade de Poincaré n-dimensional sobre um subgrupo comensurável com um subgrupo de dualidade de Poincaré $(n-1)$ dimensional $S$ dado. De fato, mostraram que nessas hipóteses a existência de uma tal decomposição depende de uma "obstrução", na forma de uma certa classe de cohomologia, denotada por $\operatorname{sing}_{G}(S)$ definida por eles. Posteriormente, tais autores em [17], generalizaram tal resultado em duas direções apresentando um resultado sobre "decomposição adaptada" de um grupo $G$ a uma família $\mathcal{S}$ de subgrupos de $G$ e sobre "decomposição simultânea". Para tanto usaram uma obstrução "sing $g_{(G, \mathcal{S})}(T)$ ", que é uma extensão da obstrução $\operatorname{sing}_{G}(S)$. Neste capítulo faremos um estudo dessas obstruções, apresentando alguns resultados cuja demonstração em alguns casos iremos omitir. As obstruções são definidas a partir de aplicações restrições, assim, no estudo da decomposição de grupos via $\operatorname{sing}_{G}(S)$, onde $G$ é um grupo e $S$ e $T$ são subgrupos de $G$, é importante explorar a relação entre o elemento não nulo de $H^{1}\left(G ; \mathcal{F}_{S}(G)\right.$ ) (domínio da aplicação $\left.\operatorname{res}_{S, \mathcal{F}_{S}(G)}^{G}\right)$ e $\mathcal{F}_{S}(G)$ classes de equivalências de elementos de $\mathcal{A}_{S}(G)=\left\{B \subset G ; B+g B \in \mathcal{F}_{S}(G), \forall g \in G\right\}$ (vide Lema 6.1.1) e no estudo da $\operatorname{sing}_{(G, \mathcal{S})}(T, S), \mathcal{S}=\left(S_{i}\right)_{i \in I}$, I finito, é importante explorar uma relação desse tipo para elementos de $H^{1}\left(G, \mathcal{S} ; \mathcal{F}_{T}(G)\right)$ (domínio de $\operatorname{res}_{G}^{(G, \mathcal{S})}$ ) e certas $I$-uplas de subconjuntos de $G$, mais explicitamente, de elementos de certo subconjunto $\mathcal{H}$ de $\left(\mathcal{A}_{T}(G)\right)^{I}$ (vide Proposição 6.2.2). Um outro resultado interessante provado é a Proposição 6.2.3. Os invariantes $\widetilde{E}(G, S)$ e $E\left(G, \mathcal{S}, \mathcal{F}_{S}(G)\right)$ bem como alguns resultados envolvendo tais invariantes, decomposição de grupos e/ou dualidade são também 
apresentados $([1],[6])$.

\subsection{Obstrução sing}

No que segue vamos supor que

$G$ é um grupo finitamente gerado, $S$ é um subgrupo de $G$ finitamente gerado, e $H^{1}\left(G ; \mathcal{F}_{S}(G)\right)$ tem dimensão 1 , isto é, $H^{1}\left(G ; \mathcal{F}_{S}(G)\right) \simeq \mathbb{Z}_{2}$.

Definição 6.1.1. Considere $\operatorname{res}_{S}^{G}: H^{1}\left(G ; \mathcal{F}_{S}(G)\right) \rightarrow H^{1}\left(S ; \mathcal{F}_{S}(G)\right)$ a aplicação restrição e $\xi$ o gerador de $H^{1}\left(G ; \mathcal{F}_{S}(G)\right)$. Definimos

$$
\operatorname{sing}_{G}(S):=\operatorname{res}_{S}^{G}(\xi) .
$$

Veremos inicialmente uma relação entre a obstrução $\operatorname{sing}_{G}(S)$ e subconjuntos $S$-quase invariantes.

Lema 6.1.1. ([16], Lema 2.2 ou [21], Lema 4.3.2, p.81) As seguintes afirmações são equivalentes:

(i) A obstrução $\operatorname{sing}_{G}(S)$ é zero ( isto é, $\left.\xi \in \operatorname{Ker}\left(\operatorname{res}_{S}^{G}\right)\right)$;

(ii) Existe um subconjunto $S$-quase invariante $B$, que não é S-finito nem S-cofinito (assim, $[B] \neq[\emptyset]$ e $[B] \neq[G]$ ) em $\mathcal{A}_{S}(G) / \mathcal{F}_{S}(G)$ tal que $S B=B$.

Demonstração: Seja $\epsilon: F \rightarrow \mathbb{Z}_{2}$ uma resolução projetiva de $\mathbb{Z}_{2}$ sobre $\mathbb{Z}_{2} G$. Então $\epsilon$ é também uma resolução de $\mathbb{Z}_{2}$ sobre $\mathbb{Z}_{2} S$.

Da sequência exata

$$
0 \rightarrow \mathcal{F}_{S}(G) \stackrel{k}{\hookrightarrow} \mathcal{P}(G) \rightarrow \frac{\mathcal{P}(G)}{\mathcal{F}_{S}(G)} \rightarrow 0
$$

obtemos o diagrama comutativo de complexos de cocadeias com linhas exatas:

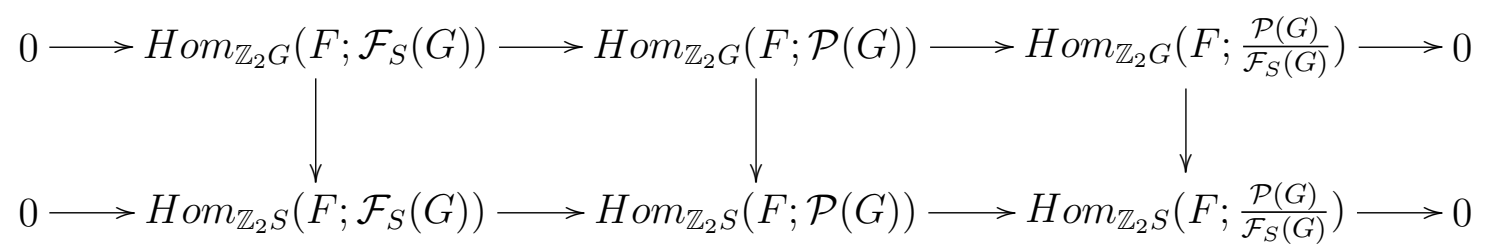

Daí, aplicando $H^{*}(-)$, obtemos o diagrama comutativo com linhas exatas:

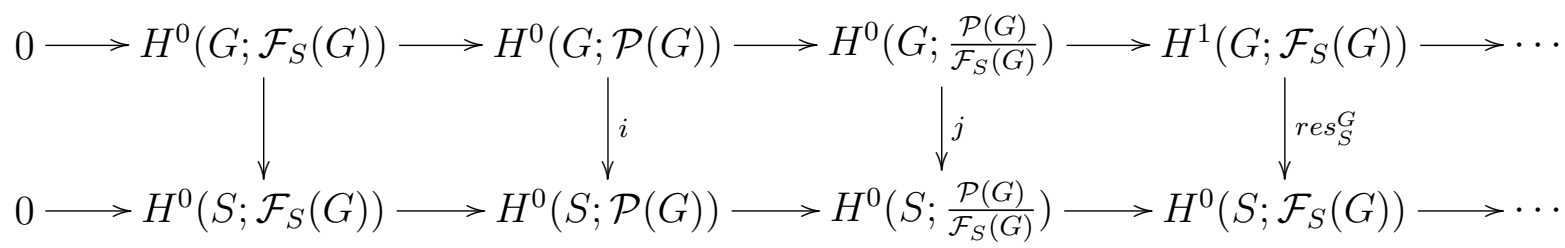


Tanto em $(i)$ como em $(i i)$ temos $[G: S]=\infty$ e daí, $H^{0}\left(G ; \mathcal{F}_{S}(G)\right)=\left(\mathcal{F}_{S}(G)\right)^{G}=$ $\left(\operatorname{Ind}_{S}^{G} \mathcal{P}(S)\right)^{G}=0$. Também temos $H^{1}(G ; \mathcal{P}(G))=0$, pelo Lema de Shapiro. Assim,

$$
\begin{aligned}
& 0 \rightarrow \mathcal{P}(G)^{G} \simeq \mathbb{Z}_{2} \stackrel{\beta}{\rightarrow}\left(\frac{\mathcal{P}(G)}{\mathcal{F}_{S}(G)}\right)^{G} \stackrel{\delta}{\rightarrow} H^{1}\left(G ; \mathcal{F}_{S}(G)\right) \rightarrow 0(*) \\
& \downarrow_{i} \quad \downarrow_{j} \quad \downarrow_{\text {res }}^{G} \\
& 0 \rightarrow\left(\mathcal{F}_{S}(G)\right)^{S} \hookrightarrow \mathcal{P}(G)^{S} \stackrel{\alpha}{\rightarrow}\left(\frac{\mathcal{P}(G)}{\mathcal{F}_{S}(G)}\right)^{S} \stackrel{\rho}{\rightarrow} H^{1}\left(S ; \mathcal{F}_{S}(G)\right) \rightarrow \cdots
\end{aligned}
$$

Da hipótese de que $H^{1}\left(G ; \mathcal{F}_{S}(G)\right) \simeq \mathbb{Z}_{2}$ e da sequência $(*)$ ser exata, segue que $\left(\frac{\mathcal{P}(G)}{\mathcal{F}_{S}(G)}\right)^{G}=\frac{\mathcal{A}_{S}(G)}{\mathcal{F}_{S}(G)}$ tem dimensão dois, e portanto tem quatro elementos. Assim existe um elemento $\left[B_{0}\right]$ em tal espaço que é diferente de $[\emptyset]=\emptyset+\mathcal{F}_{S}(G)$ e de $[G]=G+$ $\mathcal{F}_{S}(G)$, o que equivale a $B_{0}$ não ser $S$-finito e nem $S$-cofinito (de modo que $\left(\frac{\mathcal{P}(G)}{\mathcal{F}_{S}(G)}\right)^{G}=$ $\left.\left\{[\emptyset],\left[B_{0}\right],\left[B_{0}^{c}\right],[G]\right\}\right)$.

Logo, se tivermos $B_{1}$ e $B_{2}$ subconjuntos $S$-quase invariantes de $G$, que não são nem $S$ finitos nem $S$-cofinitos, então $\left[B_{1}\right]=\left[B_{2}\right]$ ou $\left[B_{1}\right]=\left[B_{2}^{c}\right]$. Assim, mostramos que existe um subconjunto $S$-quase invariante $B_{0}$ de $G$ que não é $S$-finito e nem $S$-cofinito, e então $\delta\left(\left[B_{0}\right]\right)$ é o elemento não trivial de $H^{1}\left(G ; \mathcal{F}_{S}(G)\right) \simeq \mathbb{Z}_{2}$, pois se $\delta\left(\left[B_{0}\right]\right)=0$ então da exatidão da sequência segue que $\left[B_{0}\right] \in \operatorname{Im} \beta=\{[\emptyset],[G]\}$, visto que $(\mathcal{P}(G))^{G}=\{\emptyset, G\} \simeq \mathbb{Z}_{2}$, o que é uma contradição.

Suponhamos que $\operatorname{sing}_{G}(S)$ é zero. Segue do diagrama que $\rho\left(j\left(\left[B_{0}\right]\right)\right)=0$, assim $j\left(\left[B_{0}\right]\right) \in \operatorname{Ker}(\rho)=\operatorname{Im}(\alpha)$. Agora, $(\mathcal{P}(G))^{S}$ é o conjunto de todos os subconjuntos $B$ de $G$ que satisfazem $S B=B$. Daí, um tal subconjunto $B$ pode ser escolhido de modo que $[B]=\alpha(B)=\left[B_{0}\right]=j\left(\left[B_{0}\right]\right)$ e então $B$ tem as propriedades requeridas.

Suponhamos agora que exista $[B] \in\left(\frac{\mathcal{P}(G)}{\mathcal{F}_{S}(G)}\right)^{G}$ tal que $[B] \neq[\emptyset],[B] \neq[G]$ e $S B=B$. $\operatorname{Como}(\mathcal{P}(G))^{G}=\{\emptyset, G\} \simeq \mathbb{Z}_{2}$ e $\operatorname{Im} \beta=\{[\emptyset],[G]\}$, então $[B] \notin \operatorname{Im} \beta=\operatorname{Ker}(\delta)$ e portanto $u:=\delta([B]) \neq 0$, com $B \in(\mathcal{P}(G))^{S}$. Daí, pela comutatividade do diagrama e exatidão da segunda sequência, temos $\operatorname{res}_{S}^{G}(u)=\operatorname{res}_{S}^{G}(\delta([B]))=(\rho \circ j)([B])=\rho([B])=(\rho \circ \alpha)([B])=$ 0, como desejado. Logo, $\operatorname{sing}_{G}(S)=0$.

Sabemos que se $T$ é comensurável a $S, \mathcal{F}_{S}(G)=\mathcal{F}_{T}(G)$, assim $H^{1}\left(G ; \mathcal{F}_{S}(G)\right)=$ $H^{1}\left(G ; \mathcal{F}_{T}(G)\right)$, deste modo, $\operatorname{sing}_{G}(S)=\operatorname{res}_{S}^{G}(\xi)$, com

$$
\operatorname{res}_{S}^{G}: H^{1}\left(G ; \mathcal{F}_{S}(G)\right) \rightarrow H^{1}\left(S ; \mathcal{F}_{S}(G)\right),
$$

ou podemos ver, substituindo $H^{1}\left(G ; \mathcal{F}_{S}(G)\right)$ por $H^{1}\left(G ; \mathcal{F}_{T} G\right)$, $\operatorname{sing}_{G}(S)=\operatorname{res}_{S}^{G}(\xi)$, com

$$
\operatorname{res}_{S}^{G}: H^{1}\left(G ; \mathcal{F}_{T}(G)\right) \rightarrow H^{1}\left(S ; \mathcal{F}_{T}(G)\right)
$$


Nesse caso as duas obstruções $\operatorname{sing}_{G}(S)$ e $\operatorname{sing}_{G}(T)$ estão fortemente relacionadas como mostra o lema seguinte:

Lema 6.1.2. ([16], Lema 2.3 ou [21], Lema 4.3.3) Se T é comensurável a S então $\operatorname{sing}_{G}(T)=0$ se, e somente se, $\operatorname{sing}_{G}(S)=0$.

Proposição 6.1.1. ([16], Lema 2.4) Se G se decompõe sobre um subgrupo comensurável com $S$ então $\operatorname{sing}_{G}(S)=0$.

Lema 6.1.3. Sejam $G$ um $P D^{n}$-grupo e $S$ um $P D^{n-1}$-subgrupo de $G$. Então o grupo de cohomologia $H^{1}\left(G ; \mathcal{F}_{S}(G)\right)$ é isomorfo a $\mathbb{Z}_{2}$ e assim a obstrução sing $\sin _{G}(S)$ está bem definida.

Demonstração: Temos que $H^{1}\left(G ; \mathcal{F}_{S}(G)\right) \simeq H_{n-1}\left(G ; \mathcal{F}_{S}(G)\right)$, pois $G$ é um $P D^{n}-$ grupo. Pelo Lema de Shapiro, $H_{n-1}\left(G ; \mathcal{F}_{S}(G)\right) \simeq H_{n-1}\left(S ; \overline{\mathbb{Z}_{2} S}\right)$, uma vez que $\mathcal{F}_{S}(G) \simeq$ $\overline{\mathbb{Z}_{2} S} \otimes_{\mathbb{Z}_{2} S} \mathbb{Z}_{2} G=\operatorname{Ind}_{S}^{G} \overline{\mathbb{Z}_{2} S}$. Como $S$ é um $P D^{n-1}$-subgrupo, $H_{n-1}\left(S ; \overline{\mathbb{Z}_{2} S}\right) \simeq H^{0}\left(S ; \overline{\mathbb{Z}_{2} S}\right)$. Deste modo, $H^{1}\left(G ; \mathcal{F}_{S}(G)\right) \simeq H^{0}\left(S ; \overline{\mathbb{Z}_{2} S}\right)=H^{0}\left(S ; H \operatorname{Hom}_{\mathbb{Z}_{2}}\left(\mathbb{Z}_{2} S, \mathbb{Z}_{2}\right)\right)=$ $\left(H o m_{\mathbb{Z}_{2}}\left(\mathbb{Z}_{2} S ; \mathbb{Z}_{2}\right)\right)^{S} \simeq H_{\text {om }} \mathbb{Z}_{2}\left(\mathbb{Z}_{2} S ; \mathbb{Z}_{2}\right) \simeq \mathbb{Z}_{2}$

Finalmente apresentamos (sem demonstração) o resultado de Kropholler e Roller sobre decomposição para grupos de dualidade de Poincaré.

Teorema 6.1.1. ([16], Teorema A) Sejam G um $P D^{n}$-grupo e $S$ um $P D^{n-1}$-subgrupo. $G$ se decompõe sobre um subgrupo comensurável com $S$ se, e somente se, $\operatorname{sing}_{G}(S)=0$.

\subsubsection{A Obstrução sing e os Invariantes $\tilde{e}(G, S)$ e $\tilde{E}(G, S)$}

O invariante $\tilde{e}(G, S)$, para $G$ um grupo e $S$ um subgrupo de $G$ foi definido por Kropholler e Roller indiretamente em [16] e estudado mais detalhadamente em [18]. O invariante $\tilde{E}(G, S)$ foi definido por Andrade-Fanti ([2], [1] e [6]).

Apresentaremos aqui a definição destes invariantes e algumas de suas propriedades relacionadas com a obstrução sing e decomposição de grupos. Para maiores detalhes e resultados adicionais ver as referências mencionadas acima.

Como veremos no que segue, a condição $H^{1}\left(G ; \mathcal{F}_{S}(G)\right) \simeq \mathbb{Z}_{2}$ é equivalente a $\tilde{e}(G, S)=2$.

Definição 6.1.2. Seja $(G, S)$ um par grupo com indice $[G: S]$ não necessariamente 
infinito. Define-se:

$$
\tilde{e}(G, S)=\operatorname{dim}_{\mathbb{Z}_{2}} H^{0}\left(G ; \frac{\mathcal{P}(G)}{\mathcal{F}_{S}(G)}\right)=\operatorname{dim}_{\mathbb{Z}_{2}}\left(\frac{\mathcal{P}(G)}{\mathcal{F}_{S}(G)}\right)^{G}
$$

Proposição 6.1.2. ([18], Lema 1.2) $S e[G: S]=\infty \operatorname{então} \tilde{e}(G, S)=1+\operatorname{dim}_{\mathbb{Z}_{2}} H^{1}\left(G ; \mathcal{F}_{S}(G)\right)$.

Corolário 6.1.1. Seja $(G, S)$ um par grupo com índice $[G: S]=\infty$. Então $H^{1}\left(G ; \mathcal{F}_{S}(G)\right) \simeq$ $\mathbb{Z}_{2}\left(\operatorname{sing}_{G}(S)\right.$ está bem definida) se, e somente se, $\tilde{e}(G, S)=2$. Em particular se $G$ é um $P D^{n}$-grupo e $S$ um $P D^{n-1}$-subgrupo então $\tilde{e}(G, S)=2$.

Apresentamos a seguir a definição de $\widetilde{E}(G, S)$ e alguns resultados em termos desse invariante.

Definição 6.1.3. Dado um par $(G, S)$, com $[G: S]=\infty$, define-se (vide $[6])$

$$
\widetilde{E}(G, S):=1+\operatorname{dim}_{\mathbb{Z}_{2}} \operatorname{Ker}\left(\operatorname{res}_{S, \mathcal{F}_{S}(G)}^{G}\right)
$$

onde $\operatorname{res}_{S, \mathcal{F}_{S}(G)}^{G}: H^{1}\left(G ; \mathcal{F}_{S}(G)\right) \rightarrow H^{1}\left(S ; \mathcal{F}_{S}(G)\right)$, é a aplicação restrição.

Pode-se verificar que, nas hipóteses acima, as condições $\operatorname{sing}_{G}(S)=0$ e $\operatorname{Ker}\left(r e s_{S}^{G}\right) \neq$ 0 são equivalentes, mais precisamente temos:

Lema 6.1.4. Se $(G, S)$ é um par grupo $\operatorname{com} H^{1}\left(G ; \mathcal{F}_{S}(G)\right) \simeq \mathbb{Z}_{2}$, então

1. $\operatorname{sing}_{G}(S)=0 \Leftrightarrow \tilde{E}(G, S)=2$,

2. $\operatorname{sing}_{G}(S) \neq 0 \Leftrightarrow \tilde{E}(G, S)=1$.

Demonstração: Temos que $[G: S]=\infty$ visto que $H^{1}\left(G ; \mathcal{F}_{S} G\right) \simeq \mathbb{Z}_{2}$, e $\tilde{E}(G, S)=$ $1+\operatorname{dim} \operatorname{Ker} \operatorname{res}_{S}^{G}$. Logo

(i) $\operatorname{sing}_{G}(S)=0 \Leftrightarrow \operatorname{Ker} \operatorname{res}_{S}^{G}=H^{1}\left(G, \mathcal{F}_{S} G\right) \simeq \mathbb{Z}_{2} \Leftrightarrow \tilde{E}(G, S)=2$.

(ii) $\operatorname{sing}_{G}(S) \neq 0 \Leftrightarrow$ Ker $\operatorname{res}_{S}^{G}=0 \Leftrightarrow \tilde{E}(G, S)=1$.

Como consequência da Proposição 6.1.1 e Teorema 6.1.1 obtem-se:

Proposição 6.1.3. Seja $(G, S)$ um par grupo satisfazendo $H^{1}\left(G ; \mathcal{F}_{S}(G)\right) \simeq \mathbb{Z}_{2}$, com $G$ e $S$ finitamente gerados. Se $G$ se decompõe sobre um subgrupo comensurável com $S$ então $\tilde{E}(G, S)=2$. Se $G$ um $P D^{n}$-grupo e $S$ um $P D^{n-1}$-subgrupo, $G$ se decompõe sobre um subgrupo comensurável com $S$ se, e somente se, $\tilde{E}(G, S)=2$. 
Exemplo 6.1.1. Se $(G, S)$ é um par grupo como nos dois casos (1) e (2) abaixo, então $G$ se decompõe sobre um subgrupo comensurável com $S$ :

(1) $G=\mathbb{Z}^{k}$ e $S=\mathbb{Z}^{k-1}, k \geq 2$;

(2) $G=(\mathbb{Z} \oplus \mathbb{Z}) \rtimes \mathbb{Z}$, onde $\theta: \mathbb{Z} \rightarrow \operatorname{Aut}(\mathbb{Z} \oplus \mathbb{Z})$ é dada por $\theta(c)(a, b)=\left[\begin{array}{ll}1 & 0 \\ 2 & 1\end{array}\right]^{c}\left[\begin{array}{l}a \\ b\end{array}\right]=$ $\left[\begin{array}{cc}1 & 0 \\ 2 c & 1\end{array}\right]\left[\begin{array}{l}a \\ b\end{array}\right]=(a, 2 c a+b)$ com a operação em $G$ definida por $((a, b), c)+\left(\left(a_{1}, b_{1}\right), c_{1}\right)=\left((a, b)+\theta(c)\left(a_{1}, b_{1}\right), c+c_{1}\right)=\left(a+a_{1}, b+b_{1}+2 c a_{1}, c+c_{1}\right)$ e $S=\{((a, b), 0) ; a, b \in \mathbb{Z}\}$.

Isto segue do exemplo anterior, uma vez que em (1) G é um $P D^{k}$-grupo e $S$ um $P D^{k-1}$ subgrupo e, em (2), G é um $P D^{3}$-grupo e $S$ é um $P D^{2}$-subgrupo ([3], Exemplos (iii) e $(v i))$.

Motivados nesse fato e considerando que que $\tilde{E}(G, S)$ foi definido sem a restrição de que $H^{1}\left(G ; \mathcal{F}_{S}(G)\right) \simeq \mathbb{Z}_{2}$ os autores (em [1]) acreditavam que fosse possível, pensando em termos de $\tilde{E}(G, S)$ e não em termos de $\operatorname{sing}_{G}(S)$, estender a primeira parte do resultado apresentado na proposição anterior. De fato isso é possível (ver teorema seguinte) que pode ser provado de maneira similar à dada em [16], pois usa dois resultados, cujas provas podem ser adaptadas ao invariante $\tilde{E}(G, S)$, retirando a hipótese de que $H^{1}\left(G ; \mathcal{F}_{S}(G)\right) \simeq$ $\mathbb{Z}_{2}([1])$.

Teorema 6.1.2. Seja $(G, S)$ um par grupo com $G$ e $S$ finitamente gerados e $[G: S]=\infty$. Se $G$ se decompõe sobre um subgrupo comensurável com $S$ então $\tilde{E}(G, S) \geq 2$. Ou equivalentemente, $\tilde{E}(G, S)=1$ implica que $G$ não se decompõe sobre nenhum subgrupo comensurável com $S$.

\subsection{A Obstrução sing Generalizada}

Dado um par grupo $(G, \mathcal{S})$ consistindo de um grupo $G$ e uma família finita não vazia $\mathcal{S}$ de subgrupos de $G$, Müller em [20], p. 223, apresentou a seguinte definição de decomposição adaptada (de um grupo $G$ sobre um subgrupo finito $T$ ).

Definição 6.2.1. Uma decomposição $G=G_{1} *_{T} G_{2}$ ou $G_{1} *_{T, t}$ de $G$ sobre $T$ é chamada adaptada ao par $(G, \mathcal{S})$ (ou família $\mathcal{S}$ ) se $T$ é finito e todo subgrupo $S$ pertencente a 
$\mathcal{S}$ é conjugado a um subgrupo de $G_{1}$ ou $G_{2}$. Se uma tal decomposição existe, diz-se simplesmente que o par $(G, \mathcal{S})$ é adaptado.

Exemplo 6.2.1. O produto livre $G=G_{1} * G_{2}$ é uma decomposição de $G$ adaptada ao par $\left(G,\left\{G_{1}, G_{2}\right\}\right)$ (tomando $T$ o subgrupo trivial).

Em [17], p. 411, Kropholler e Roller estenderam esta definição por considerar $T$ n $\tilde{o} o$ necessariamente finito, $(G, \mathcal{S})$ um $P D^{n}$-par, com $\mathcal{S}$ uma família (finita) de subgrupos de G, e T um $P D^{n-1}$ - subgrupo e, apresentaram sob certas hipóteses de dualidade, alguns resultados sobre decomposição de grupos para pares grupo $(G, \mathcal{S})$. Para tanto usaram uma obstrução "sing $(G, \mathcal{S})(T, S)$ ", que estende a obstrução $\operatorname{sing}_{G}(S)$ referida anteriormente (se consideramos $\mathcal{S}=\emptyset$ e $T=S$ ). Um dos principais resultados apresentados foi:

Teorema 6.2.1. ([17] Teorema $A$, p.401) Seja $(G, \mathcal{S})$ um $P D^{n}$-par e $T$ um $P D^{n-1}$ subgrupo de $G$, tal que $T$ não é comensurável com nenhum conjugado de $S_{i}$, para todo $S_{i}$ em $\mathcal{S}$. Então $G$ admite uma decomposição adaptada a $\mathcal{S}$, sobre um subgrupo comensurável com $T$ se, e somente se, $\operatorname{sing}_{(G, \mathcal{S})}(T, T) \stackrel{\text { not. }}{=} \operatorname{sing}_{(G, \mathcal{S})}(T)=0$.

Nosso objetivo é apresentar a definição da obstrução "sing generalizada" para pares grupo, e apresentar alguns dos resultados de [17] relacionados a tal obstrução e que são úteis para a prova do resultado acima.

Vejamos primeiramente a definição de uma sing "mista".

Definição 6.2.2. Sejam $G$ um grupo, $S$ e $T$ subgrupos de $G$ e $\operatorname{res}_{S}^{G}: H^{1}\left(G ; \mathcal{F}_{T}(G)\right) \rightarrow$ $H^{1}\left(S ; \mathcal{F}_{T}(G)\right)$, a aplicação restrição. Se $H^{1}\left(G ; \mathcal{F}_{T}(G)\right) \simeq \mathbb{Z}_{2}$, com gerador $\xi$, então definimos a sing mista $\operatorname{sing}_{G}(S, T):=\operatorname{res}_{S, \mathcal{F}_{T}(G)}^{G}(\xi)$.

Lema 6.2.1. (a) $\operatorname{sing}_{G}(S)=\operatorname{sing}_{G}(S, S)$.

(b) Se $S$ e $T$ são subgrupos comensuráveis de $G$ então $\operatorname{sing}_{G}(S, T)=\operatorname{sing}_{G}(S)$, $\operatorname{sing}_{G}(T, S)=\operatorname{sing}_{G}(T)$, e $\operatorname{sing}_{G}(S, T)=0$ se, e somente se, $\operatorname{sing}_{G}(T, S)=0$.

Demonstração A parte $(a)$ é óbvia e a parte $(b)$ segue do fato que para $S$ e $T$ comensuráveis tem-se $\mathcal{F}_{T}(G)=\mathcal{F}_{S}(G)$ e $\operatorname{sing}_{G}(S)=0$ se, e somente se, $\operatorname{sing}_{G}(T)=0$.

Definição 6.2.3. (sing generalizada) Sejam $(G, \mathcal{S})$ um par grupo, e $S$ e $T$ subgrupos de $G \operatorname{com} H^{1}\left(G, \mathcal{S} ; \mathcal{F}_{T}(G)\right) \simeq \mathbb{Z}_{2}$. Seja $\zeta$ o único elemento não nulo de $H^{1}\left(G, \mathcal{S} ; \mathcal{F}_{T}(G)\right)$, então definimos 


$$
\operatorname{sing}_{(G, \mathcal{S})}(T, S)=\operatorname{res}_{S}^{G}\left(\operatorname{res}_{G}^{(G, \mathcal{S})}(\zeta)\right)
$$

a imagem de $\zeta$ pela composta das aplicações restrições

$$
H^{1}\left(G, \mathcal{S} ; \mathcal{F}_{T}(G)\right) \stackrel{\operatorname{res}_{G}^{(G, \mathcal{S})}}{\longrightarrow} H^{1}\left(G ; \mathcal{F}_{T}(G)\right) \stackrel{\operatorname{res}_{S}^{G}}{\longrightarrow} H^{1}\left(S ; \mathcal{F}_{T}(G)\right) .
$$

Observação 6.2.1. $\quad$ 1. Se $S=T$, denotamos $\operatorname{sing}_{(G, \mathcal{S})}(T, T)$ simplesmente por $\operatorname{sing}_{(G, \mathcal{S})}(T)$.

2. Uma vez que $H^{1}\left(G, \emptyset ; \mathcal{F}_{T}(G)\right)=H^{1}\left(G ; \mathcal{F}_{T}(G)\right)$ (por convenção), segue que $\operatorname{sing}_{(G, \emptyset)}(S, T)=\operatorname{sing}_{G}(S, T)$.

3. $\operatorname{sing}_{G}(S)=\operatorname{sing}_{(G, \emptyset)}(S, S) \stackrel{\text { not. }}{=} \operatorname{sing}_{(G, \emptyset)}(S)$.

Lema 6.2.2. Sejam $(G, \mathcal{S})$ um par grupo, $S, T$ subgrupos de $G$, e suponhamos $H^{1}\left(G, \mathcal{S} ; \mathcal{F}_{T}(G)\right) \simeq \mathbb{Z}_{2}$.

1. Se $T^{*}$ é um subgrupo comensurável com $T$ então $\operatorname{sing}_{(G, \mathcal{S})}\left(T^{*}, S\right)=\operatorname{sing}_{(G, \mathcal{S})}(T, S)$.

2. Se $S_{1}$ é um subgrupo de $S$ com $\left[S: S_{1}\right]<\infty$ então $\operatorname{sing}_{(G, \mathcal{S})}(T, S)=0 \Rightarrow$ $\operatorname{sing}_{(G, \mathcal{S})}\left(T, S_{1}\right)=0$.

\section{Demonstração:}

1. Sabemos que se $T^{*}$ é comensurável com $T$ então $\mathcal{F}_{T}(G)=\mathcal{F}_{T^{*}}(G)$. Deste modo, $\operatorname{comosing}_{(G, \mathcal{S})}\left(T^{*}, S\right)=\left(\operatorname{res}_{S}^{G}\right.$ ores $\left._{G}^{(G, \mathcal{S})}\right)(\zeta)$, onde $\operatorname{res}_{S}^{G}$ ores ${ }_{G}^{(G, \mathcal{S})}: H^{1}\left(G, \mathcal{S} ; \mathcal{F}_{T^{*}}(G)\right) \rightarrow$ $H^{1}\left(S ; \mathcal{F}_{T^{*}}(G)\right)$, segue claramente que $\operatorname{sing}_{(G, \mathcal{S})}\left(T^{*}, S\right)=\operatorname{sing}_{(G, \mathcal{S})}(T, S)$.

2. Segue do fato que $\operatorname{res}_{S_{1}}^{G}=\operatorname{res}_{S_{1}}^{S} \circ \operatorname{res}_{S}^{G}$

$$
\begin{gathered}
H^{1}\left(G ; \mathcal{F}_{T}(G)\right) \stackrel{\operatorname{res}_{S}^{G}}{\rightarrow} H^{1}\left(S ; \mathcal{F}_{T}(G)\right) \stackrel{\operatorname{res}_{S_{1}}^{S}}{\rightarrow} H^{1}\left(S_{1} ; \mathcal{F}_{T}(G)\right), \\
\text { e } \operatorname{assim} \operatorname{sing}_{(G, \mathcal{S})}\left(T, S_{1}\right)=\operatorname{res}_{S_{1}}^{G}\left(\operatorname{res}_{G}^{(G, \mathcal{S})}(\zeta)\right)=\left(\operatorname{res}_{S_{1}}^{S} \circ \operatorname{res}_{S}^{G}\right)\left(\operatorname{res}_{G}^{(G, \mathcal{S})}\right)(\zeta)= \\
\operatorname{res}_{S_{1}}^{S}\left(\operatorname{res}_{S}^{G}\left(\operatorname{res}_{G}^{(G, \mathcal{S})}(\zeta)\right)\right)=\operatorname{res}_{S_{1}}^{S}\left(\operatorname{sing}_{(G, \mathcal{S})}(T, S)\right)=\operatorname{res}_{S_{1}}^{S}(0)=0 .
\end{gathered}
$$

Vamos fixar $G$ um grupo e $S$ e $T$ subgrupos de $G$. No estudo da decomposição de grupos via $\operatorname{sing}_{G}(S)=\operatorname{sing}_{(G, \emptyset)}(S)$ foi importante explorar a relação entre o elemento não nulo de $H^{1}\left(G ; \mathcal{F}_{S}(G)\right)$ (domínio da aplicação $\left.\operatorname{res}_{S, \mathcal{F}_{S}(G)}^{G}\right)$ e $\mathcal{F}_{S}(G)$ - classes de equivalências de elementos de $\mathcal{A}_{S}(G)$ (vide Lema 6.1.1). No estudo da $\operatorname{sing}_{(G, \mathcal{S})}(T, S)$ $\mathcal{S}=\left(S_{i}\right)_{i \in I}, I$ finito, também é importante explorar uma relação desse tipo para elementos de $H^{1}\left(G, \mathcal{S} ; \mathcal{F}_{T}(G)\right.$ ) (domínio de $\operatorname{res}_{G}^{(G, \mathcal{S})}$ ) e certas $I$-uplas de subconjuntos de $G$, mais explicitamente, de elementos de certo subconjunto $\mathcal{H}$ de $\left(\mathcal{A}_{T}(G)\right)^{I}$. Vejamos inicialmente uma relação entre elementos de $H^{1}\left(G ; \mathcal{F}_{T}(G)\right.$ ) (domínio da aplicação $\operatorname{res}_{S, \mathcal{F}_{T}(G)}^{G}$ ) e $\mathcal{F}_{T}(G)$ classes de equivalências de elementos $\mathcal{A}_{T}(G)$, que é, de certo modo, uma extensão do Lema 6.1.1, envolvendo agora dois subgrupos $S$ e $T$ de $G$. 
Lema 6.2.3. ([17], Lema 3.1) Sejam $T, S$ subgrupos de $G$ tal que $[G: T]=\infty$, e $[B] \in \mathcal{A}_{T}(G) / \mathcal{F}_{T}(G)$. Então existe uma sequência exata curta,

$$
0 \longrightarrow \mathbb{Z}_{2} \longrightarrow \mathcal{A}_{T}(G) / \mathcal{F}_{T}(G) \stackrel{\delta}{\longrightarrow} H^{1}\left(G ; \mathcal{F}_{T}(G)\right) \longrightarrow 0
$$

e $\delta([B])$ pertence ao kernel da aplicação restrição $\operatorname{res}_{S}^{G}: H^{1}\left(G ; \mathcal{F}_{T}(G)\right) \rightarrow H^{1}\left(S ; \mathcal{F}_{T}(G)\right)$ se, e somente se existe $B_{0} \in \mathcal{A}_{T}(G)$ tal que $B_{0}+B$ é $T$-finito e $S B_{0}=B_{0}$.

Demonstração: Considere a seguinte sequência exata de $\mathbb{Z}_{2} G$-módulos

$$
0 \longrightarrow \mathcal{F}_{T}(G) \longrightarrow \mathcal{P}(G) \longrightarrow \mathcal{P}(G) / \mathcal{F}_{T}(G) \longrightarrow 0
$$

Aplicando o funtor $H^{*}(G ;-)$ a essa sequência obteremos a sequência exata longa em cohomologia

$0 \rightarrow H^{0}\left(G ; \mathcal{F}_{T}(G)\right) \rightarrow H^{0}(G ; \mathcal{P}(G)) \rightarrow H^{0}\left(G ; \mathcal{P}(G) / \mathcal{F}_{T}(G)\right) \stackrel{\delta}{\rightarrow} H^{1}\left(G ; \mathcal{F}_{T}(G)\right) \rightarrow$ $H^{1}(G ; \mathcal{P}(G)) \rightarrow \cdots$

Agora observando que

$H^{0}\left(G ; \mathcal{F}_{T}(G)\right)=\left(\mathcal{F}_{T}(G)\right)^{G}=\left(\operatorname{Ind}_{T}^{G} \mathcal{P}(T)\right)^{G}=0$, pois $[G: T]=\infty ;$

$H^{0}(G ; \mathcal{P}(G)) \simeq \mathbb{Z}_{2}$, e

$H^{1}(G ; \mathcal{P}(G))=0$ (ver Exemplo 4.1.1 (aplicação do Lema de Shapiro)), e substituindo na sequência anterior obteremos a sequência desejada.

Para a segunda parte do Lema considere o seguinte diagrama comutativo, onde as linhas exatas são obtidas da sequência (6.1) aplicando os funtores $H^{*}(G ;-)$ e $H^{*}(S ;-)$ respectivamente,

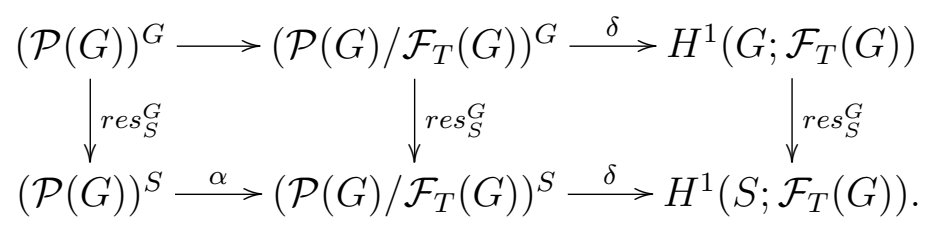

Suponha por hipótese que $\delta([B]) \in \operatorname{Ker}\left(\operatorname{res}_{S}^{G}\right)$, isto é, $\operatorname{res}_{S}^{G}(\delta([B]))=0$. Da comutatividade do diagrama temos $\delta\left(\operatorname{res}_{S}^{G}([B])\right)=0$, ou seja, $\operatorname{res}_{S}^{G}([B]) \in \operatorname{Ker}(\delta)$.

Como as sequências do diagrama são exatas segue que $[B]=\operatorname{res}_{S}^{G}([B]) \in \operatorname{Im}(\alpha)=$ $\operatorname{Ker}(\delta)$. Sendo assim, existe $B_{0} \in(\mathcal{P}(G))^{S}$ tal que $\alpha\left(B_{0}\right)=[B]$.

Agora observe que

1. $\alpha\left(B_{0}\right)=B_{0}+\mathcal{F}_{T}(G)=[B]=B+\mathcal{F}_{T}(G) \Rightarrow B_{0}+B \in \mathcal{F}_{T}(G)$,

2. $B_{0} \in(\mathcal{P}(G))^{S}=\{A \in \mathcal{P}(G) ; s A=A, \forall s \in S\} \Rightarrow S B_{0}=B_{0}$,

3. $B_{0} \in \mathcal{A}_{T}(G)$, pois $B_{0}+B \in \mathcal{F}_{T}(G) \Rightarrow B_{0}+B=X \in \mathcal{F}_{T}(G) \subset \mathcal{A}_{T}(G) \Rightarrow B_{0}=$ $B+X \in \mathcal{A}_{T}(G)$, visto que $B \in \mathcal{A}_{T}(G)$.

Portanto existe $B_{0} \in \mathcal{A}_{T}(G)$ satisfazendo todas as propriedades desejadas. 
Reciprocamente, supondo que existe $B_{0} \in \mathcal{A}_{T}(G)$ tal que $B_{0}+B \in \mathcal{F}_{T}(G)$ e $S B_{0}=B_{0}$, temos que

$$
\begin{aligned}
& B_{0}+B \in \mathcal{F}_{T}(G) \Rightarrow\left[B_{0}\right]=[B] \text { e } S B_{0}=B_{0} \Rightarrow B_{0} \in(\mathcal{P}(G))^{S}, \\
& B_{0} \in(\mathcal{P}(G))^{S} \Rightarrow \alpha\left(B_{0}\right)=B_{0}+\mathcal{F}_{T}(G)=B+\mathcal{F}_{T}(G)=[B]=\operatorname{res}_{S}^{G}([B]) .
\end{aligned}
$$

Como as sequências do diagrama são exatas temos $\delta \circ \alpha=0$, e deste modo $0=\delta\left(\alpha\left(B_{0}\right)\right)=0 \Rightarrow \delta\left(\operatorname{res}_{S}^{G}([B])\right) \stackrel{\text { d.comut. }}{=} \operatorname{res}_{S}^{G}(\delta([B]))=0 \Rightarrow \delta([B]) \in \operatorname{ker}\left(\operatorname{res}_{S}^{G}\right)$.

Fixemos uma família finita e não vazia, $\mathcal{S}=\left(S_{i}\right)_{i \in I}$, de subgrupos de $G$. Como já mencionado queremos encontrar uma interpretação para os elementos de $H^{1}\left(G, \mathcal{S} ; \mathcal{F}_{T}(G)\right)$, mas agora em termos de certas $I$-uplas de subconjuntos de $G$. Para tanto precisamos entender melhor o comportamento da aplicação

$$
\operatorname{res}_{G}^{(G, \mathcal{S})}: H^{1}\left(G, \mathcal{S} ; \mathcal{F}_{T}(G)\right) \rightarrow H^{1}\left(G ; \mathcal{F}_{T}(G)\right) .
$$

De fato, mostraremos que $\operatorname{res}_{G}^{(G, \mathcal{S})}$ é a composta de duas aplicações. Isto será uma consequência (corolário) da proposição seguinte:

Proposição 6.2.1. Seja $\tau: \mathbb{Z}_{2} G \rightarrow \mathbb{Z}_{2}(G / \mathcal{S})$ uma $\mathbb{Z}_{2} G$-aplicação preservando aumentação $e$ considere as aplicações aumentação $\varepsilon: \mathbb{Z}_{2}(G / \mathcal{S}) \rightarrow \mathbb{Z}_{2}$ e $\in: \mathbb{Z}_{2} G \rightarrow \mathbb{Z}_{2}$, tais que $\Delta=\operatorname{Ker}(\varepsilon)$ e $\mathcal{G}=\operatorname{Ker}(\epsilon) . \quad$ Então $\tau$ induz uma aplicação $\tau^{*}: \operatorname{Hom}_{\mathbb{Z}_{2} G}(\Delta, M) \rightarrow$ $\operatorname{Hom}_{\mathbb{Z}_{2} G}(\mathcal{G}, M)$, tal que $\tau^{*}(h)=\left.h \circ \tau\right|_{\mathcal{G}}$ e a composição

$$
\operatorname{Hom}_{\mathbb{Z}_{2} G}(\Delta, M) \stackrel{\tau^{*}}{\rightarrow} \operatorname{Hom}_{\mathbb{Z}_{2} G}(\mathcal{G}, M) \stackrel{\rho}{\rightarrow} H^{i}(G ; M),
$$

( $\rho$ sendo o homomorfismo conexão) é a aplicação $\operatorname{res}_{G}^{(G, \mathcal{S})}: H^{1}(G, \mathcal{S} ; M) \rightarrow H^{1}(G ; M)$, para todo $\mathbb{Z}_{2} G$-módulo $M$.

Demonstração: Primeiro observamos que se $\tau$ preserva aumentação então $\varepsilon \circ \tau=\epsilon$. Logo, $u \in \operatorname{Ker}(\epsilon) \Leftrightarrow \tau(u) \in \operatorname{Ker}(\varepsilon)$. Assim temos bem definida a aplicação $\left.\tau\right|_{\mathcal{G}}: \mathcal{G} \rightarrow \Delta$ e consequentemente está bem definida a aplicação

$$
\tau^{*}: \operatorname{Hom}_{\mathbb{Z}_{2} G}(\Delta, M) \rightarrow H_{\mathrm{O}_{2}}(\mathcal{G}, M) ; \tau^{*}(h)=\left.h \circ \tau\right|_{\mathcal{G}} .
$$

Mostremos a segunda afirmação.

Seja $M$ um $\mathbb{Z}_{2} G$-módulo. Como vimos podemos identificar, a menos de isomorfismo, $H^{1}(G, \mathcal{S} ; M)$ com $\operatorname{Hom}_{\mathbb{Z}_{2} G}(\Delta, M)$, e seja

$$
\operatorname{res}_{G}^{(G, \mathcal{S})}: H^{1}(G, \mathcal{S} ; M) \equiv \operatorname{Hom}_{\mathbb{Z}_{2} G}(\Delta, M) \rightarrow H^{1}(G ; M)
$$

o homomorfismo da sequência exata longa (vide Teorema 2.3.1)

$$
0 \rightarrow H^{0}(G ; M) \rightarrow H^{0}(\mathcal{S} ; M) \rightarrow H^{0}\left(G ; \operatorname{Hom}_{\mathbb{Z}_{2}}(\Delta, M)\right) \stackrel{\operatorname{res}_{G}^{(G, \mathcal{S})}}{\rightarrow} H^{1}(G ; M) \rightarrow \cdots
$$

obtida a menos de isomorfismos por aplicar $H^{*}(G ;-)$ a sequência exata curta

$$
0 \rightarrow \operatorname{Hom}_{\mathbb{Z}_{2}}\left(\mathbb{Z}_{2}, M\right) \equiv M \stackrel{\varepsilon^{\#}}{\rightarrow} \operatorname{Hom}_{\mathbb{Z}_{2}}\left(\mathbb{Z}_{2}(G / \mathcal{S}), M\right) \stackrel{i^{\#}}{\rightarrow} H_{o m} \mathbb{Z}_{2}(\Delta, M) \rightarrow 0
$$

(que foi obtida a partir da sequência $0 \rightarrow \Delta \stackrel{i}{\rightarrow} \mathbb{Z}_{2}(G / \mathcal{S}) \stackrel{\varepsilon}{\rightarrow} \mathbb{Z}_{2} \rightarrow 0$ ).

Considere o seguinte diagrama: 


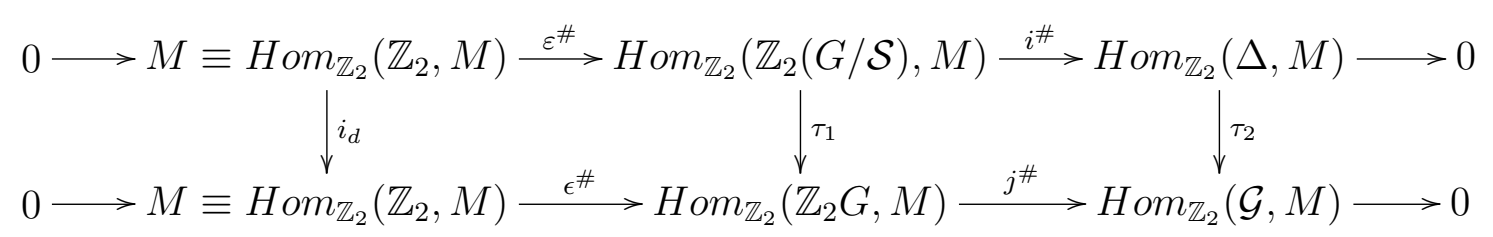

onde a sequência de baixo é obtida a partir da sequência exata curta:

$$
0 \rightarrow \mathcal{G} \stackrel{j}{\rightarrow} \mathbb{Z}_{2} G \stackrel{\epsilon}{\rightarrow} \mathbb{Z}_{2} \rightarrow 0
$$

com $j$ é a inclusão, e as aplicações verticais são dadas por $i d, \tau_{1}(h):=h \circ \tau$ e $\tau_{2}(f):=\left.f \circ \tau\right|_{\mathcal{G}}$.

É fácil verificar que o diagrama acima é comutativo, ou seja, $\tau_{1} \circ \varepsilon^{\#}=\epsilon^{\#}$ e $\tau_{2} \circ i^{\#}=$ $j^{\#} \circ \tau_{1}$.

Daí, pela naturalidade do homomorfismo conexão ( " $\rho^{n}: H^{n}\left(G ; M_{1}\right) \rightarrow H^{n+1}\left(G ; M_{1}^{\prime}\right)$ ", vide Proposição 2.2.2) segue que o quadrado seguinte é comutativo:

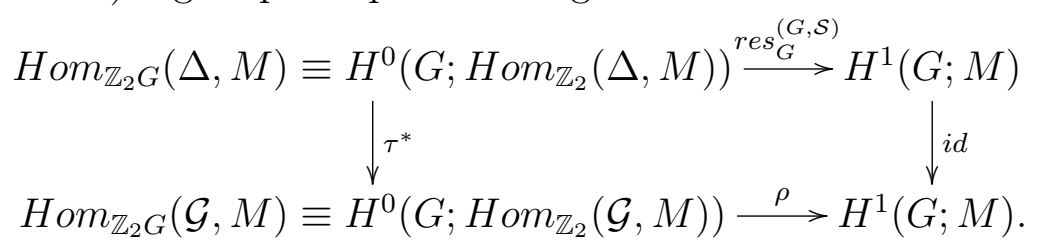

e assim $\operatorname{res}_{G}^{(G, \mathcal{S})}$ é a composição desejada, onde $\rho$ é o homomorfismo conexão para a sequência exata longa induzida da sequência (2) acima por aplicar $H^{*}(G ;-)$ :

$0 \rightarrow H^{0}(G ; M) \rightarrow H^{0}\left(G ; H \operatorname{Hom}_{\mathbb{Z}_{2}}\left(\mathbb{Z}_{2} G, M\right)\right) \rightarrow H^{0}\left(G ; H \operatorname{Hom}_{\mathbb{Z}_{2}}(\mathcal{G}, M)\right) \stackrel{\rho}{\rightarrow} H^{1}(G ; M) \rightarrow \cdots$, e $\tau^{*}(h)=\left.h \circ \tau\right|_{\mathcal{G}}$.

Corolário 6.2.1. Suponhamos $\mathcal{S}=\left(S_{i}\right)_{i \in I}$ uma família finita de subgrupos de $G$. Escolha um elemento $i \in I$, por exemplo $i=1$, e seja $\tau: \mathbb{Z}_{2} G \rightarrow \mathbb{Z}_{2}(G / \mathcal{S})$ definida nos geradores por $\tau(g)=g S_{1}$ e estendida por linearidade. Considere $T$ um subgrupo de $G$. Então $\tau$ é uma $\mathbb{Z}_{2} G$-aplicação preservando aumentação, e res ${ }_{G}^{(G, \mathcal{S})}: H^{1}\left(G, \mathcal{S} ; \mathcal{F}_{T}(G)\right) \rightarrow$ $H^{1}\left(G ; \mathcal{F}_{T}(G)\right)$ é a composição $\operatorname{Hom}_{\mathbb{Z}_{2} G}\left(\Delta, \mathcal{F}_{T}(G)\right) \stackrel{\tau *}{\rightarrow} \operatorname{Hom}_{\mathbb{Z}_{2} G}\left(\mathcal{G}, \mathcal{F}_{T}(G)\right) \stackrel{\rho}{\rightarrow} H^{1}\left(G ; \mathcal{F}_{T}(G)\right)$, sendo $\tau^{*}(h)=\left.h \circ \tau\right|_{\mathcal{G}}$

Demonstração: É fácil verificar que $\tau$ é um $\mathbb{Z}_{2} G$-aplicação.

Agora, dado um elemento gerador $1 . g \in \mathbb{Z}_{2} G$, tem-se: $(\varepsilon \circ \tau)(1 . g)=\varepsilon\left(g S_{1}\right)=1=$ $\epsilon(1 . g)$, donde segue que $\tau$ preserva aumentação. Assim basta aplicar o resultado anterior para $\tau$ e $M=\mathcal{F}_{T}(G)$.

Seja $(G, \mathcal{S})$ um par grupo, com $\mathcal{S}=\left(S_{i}\right)_{i \in I}, I$ finito, e considere

$$
(\mathcal{P}(G))^{I}=\underbrace{\mathcal{P}(G) \times \cdots \times \mathcal{P}(G)}_{I \text { vezes }}
$$

sendo um elemento $\mathbf{A} \in(\mathcal{P}(G))^{I}$ da forma $\left(A_{i}\right)_{i \in I}$, com $A_{i} \in \mathcal{P}(G)$, para todo $i \in I$. Agora estamos em condições de estabelecer uma relação entre elementos de $H^{1}\left(G, \mathcal{S} ; \mathcal{F}_{T}(G)\right)$ e 
certos elementos de $\left(\mathcal{A}_{T}(G)\right)^{I}$.

Para $\mathbf{A}=\left(A_{i}\right)_{i \in I}$ e $\mathbf{B}=\left(B_{i}\right)_{i \in I}$ pertencentes a $(\mathcal{P}(G))^{I}$ definimos as operações usuais, $g \mathbf{B}=g\left(B_{i}\right)_{i \in I}=\left(g B_{i}\right)_{i \in I}$,

$\mathbf{B}^{c}=\left(B_{i}^{c}\right)_{i \in I}$,

$\mathbf{A}+\mathbf{B}=\left(A_{i}\right)_{i \in I}+\left(B_{i}\right)_{i \in I}=\left(A_{i}+B_{i}\right)_{i \in I}$,

$\mathbf{A} \cap \mathbf{B}=\left(A_{i}\right)_{i \in I} \cap\left(B_{i}\right)_{i \in}=\left(A_{i} \cap B_{i}\right)_{i \in I}$,

$\mathbf{A} \subseteq \mathbf{B}$ se, e somente se $A_{i} \subseteq B_{i}, \forall i \in I$.

Escolha um elemento $i \in I$, por exemplo $i=1$.

Proposição 6.2.2. ([17]], Lema 3.2) Seja $\mathcal{H}=\left\{\boldsymbol{B} \in\left(\mathcal{A}_{T}(G)\right)^{I} ; B_{i}+B_{j} \in \mathcal{F}_{T}(G)\right.$ e $S_{i} B_{i}=B_{i}$ para todo $\left.i, j \in I\right\}$. Então:

(1) Existe uma sequência exata curta

$$
0 \longrightarrow \mathbb{Z}_{2} \longrightarrow \mathcal{H} \stackrel{\delta}{\longrightarrow} H^{1}\left(G, \mathcal{S} ; \mathcal{F}_{T}(G)\right) \longrightarrow 0
$$

(2) Se $\boldsymbol{B}=\left(B_{i}\right)_{i \in I} \in \mathcal{H}$ representa um elemento $\zeta$ de $H^{1}\left(G, \mathcal{S} ; \mathcal{F}_{T}(G)\right)$, mais precisamente, se $\delta(\boldsymbol{B})=\zeta$ então $\operatorname{res}_{G}^{(G, \mathcal{S})}(\zeta) \in H^{1}\left(G ; \mathcal{F}_{T}(G)\right)$ é representado por $B_{1}$, ou seja, $\delta\left(\left[B_{1}\right]\right)=\operatorname{res}_{G}^{(G, \mathcal{S})}(\zeta)$, onde $\delta$ é a aplicação dada na sequência do Lema 6.2.3, $\operatorname{eres}_{G}^{(G, \mathcal{S})}: H^{1}\left(G, \mathcal{S} ; \mathcal{F}_{T}(G)\right) \longrightarrow H^{1}\left(G ; \mathcal{F}_{T}(G)\right)$.

\section{Demonstração:}

(1) Considere a sequência exata longa para o par $(G, \mathcal{S})$ com coeficientes em $\mathcal{P}(G)$,

$$
0 \rightarrow H^{0}(G ; \mathcal{P}(G)) \rightarrow H^{0}(\mathcal{S} ; \mathcal{P}(G)) \stackrel{\delta}{\rightarrow} H^{1}(G, \mathcal{S} ; \mathcal{P}(G)) \rightarrow H^{1}(G ; \mathcal{P}(G)) \rightarrow \cdots
$$

Sabemos que $H^{0}(G ; \mathcal{P}(G)) \simeq \mathbb{Z}_{2}$ e $H^{1}(G ; \mathcal{P}(G))=0$, donde obtem-se a sequência exata

$$
0 \longrightarrow \mathbb{Z}_{2} \longrightarrow H^{0}(\mathcal{S} ; \mathcal{P}(G)) \stackrel{\delta}{\longrightarrow} H^{1}(G, \mathcal{S} ; \mathcal{P}(G)) \longrightarrow 0
$$

Ainda

- $H^{0}(\mathcal{S} ; \mathcal{P}(G)) \stackrel{\text { not. }}{=} \prod_{i \in I} H^{0}\left(S_{i} ; \mathcal{P}(G)\right)=\prod_{i \in I}(\mathcal{P}(G))^{S_{i}} \simeq \operatorname{Hom}_{\mathbb{Z}_{2} G}\left(\mathbb{Z}_{2}(G / \mathcal{S}), \mathcal{P}(G)\right)$ (pois $\phi: \operatorname{Hom}_{\mathbb{Z}_{2} G}\left(\mathbb{Z}_{2}(G / \mathcal{S}), \mathcal{P}(G)\right) \longrightarrow \prod_{i \in I}(\mathcal{P}(G))^{S_{i}} ; f \mapsto\left(f\left(S_{i}\right)\right)_{i \in I}$ é um isomorfismo, com inversa $\phi^{-1}: \prod_{i \in I}(\mathcal{P}(G))^{S_{i}} \rightarrow \operatorname{Hom}_{\mathbb{Z}_{2} G}\left(\mathbb{Z}_{2}(G / \mathcal{S}), \mathcal{P}(G)\right) ; \phi^{-1}\left(B_{1}, \ldots, B_{n}\right)=$ $\left.f, \operatorname{com} f\left(S_{i}\right):=B_{i}, i \in I\right)$.

- $H^{1}(G, \mathcal{S} ; \mathcal{P}(G)):=H^{0}\left(G, \operatorname{Hom}_{\mathbb{Z}_{2}}(\Delta, M)\right)=\left(\operatorname{Hom}_{\mathbb{Z}_{2}}(\Delta, M)\right)^{G} \simeq H_{\mathrm{Hom}_{2} G}(\Delta, \mathcal{P}(G))$, onde $\Delta$ é o Kernel da aplicação aumentação $\varepsilon: \mathbb{Z}_{2}(G / \mathcal{S}) \rightarrow \mathbb{Z}_{2}$. 
- Podemos considerar $H^{1}\left(G, \mathcal{S} ; \mathcal{F}_{T}(G)\right)$ como um submódulo de $H^{1}(G, \mathcal{S} ; \mathcal{P}(G))$, pois $H^{1}\left(G, \mathcal{S} ; \mathcal{F}_{T}(G)\right) \simeq H_{\operatorname{om}_{\mathbb{Z}_{2} G}}\left(\Delta, \mathcal{F}_{T}(G)\right) \subset \operatorname{Hom}_{\mathbb{Z}_{2} G}(\Delta, \mathcal{P}(G)) \simeq H^{1}(G, \mathcal{S} ; \mathcal{P}(G))$. A inclusão segue do fato que $\mathcal{F}_{T}(G) \subset \mathcal{P}(G)$.

- A menos de isomorfismo, podemos ver o homomorfismo $\delta$ da sequência (6.2) como a aplicação $\varphi: \operatorname{Hom}_{\mathbb{Z}_{2} G}\left(\mathbb{Z}_{2}(G / \mathcal{S}), \mathcal{P}(G)\right) \rightarrow H_{o m} \mathbb{Z}_{2} G(\Delta, \mathcal{P}(G)) ;\left.h \mapsto h\right|_{\Delta}$. Notemos que, como nesse caso $\delta$ é sobrejetora (pois a sequência (6.3) é exata), então $\varphi$ também será sobrejetora.

Tome $\mathcal{K}=\phi\left(\varphi^{-1}\left(\operatorname{Hom}_{\mathbb{Z}_{2} G}\left(\Delta, \mathcal{F}_{T}(G)\right)\right)\right) \subset H^{0}(\mathcal{S} ; \mathcal{P}(G))=\prod_{i \in I}(\mathcal{P}(G))^{S_{i}} \subset(\mathcal{P}(G))^{I}$.

Essas condições estão indicadas no diagrama abaixo:

$$
\begin{aligned}
& 0 \longrightarrow \mathbb{Z}_{2} \longrightarrow H^{0}(\mathcal{S} ; \mathcal{P}(G)) \stackrel{\delta}{\longrightarrow} H^{1}(G, \mathcal{S} ; \mathcal{P}(G)) \longrightarrow 0 \\
& \simeq \uparrow \phi \quad \downarrow \simeq \\
& \operatorname{Hom}_{\mathbb{Z}_{2} G}\left(\mathbb{Z}_{2}(G / \mathcal{S}), \mathcal{P}(G)\right) \stackrel{\varphi}{\rightarrow} \operatorname{Hom}_{\mathbb{Z}_{2} G}(\Delta, \mathcal{P}(G)) \\
& \mathcal{K} \rightarrow \operatorname{Hom}_{\mathbb{Z}_{2} G}\left(\Delta, \mathcal{F}_{T}(G)\right) \simeq H^{1}\left(G, \mathcal{S} ; \mathcal{F}_{T}(G)\right)
\end{aligned}
$$

Afirmamos que $\mathcal{K}=\mathcal{H}$. De fato:

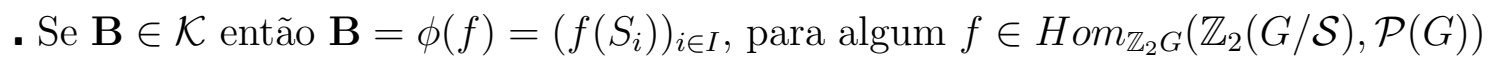
$\operatorname{com} \varphi(f)=\left.f\right|_{\Delta} \in \operatorname{Hom}_{\mathbb{Z}_{2} G}\left(\Delta, \mathcal{F}_{T}(G)\right)$, e além disso temos

1. $B_{i}+g B_{i}=f\left(S_{i}\right)+g f\left(S_{i}\right)=f\left(S_{i}\right)+f\left(g S_{i}\right)=f\left(S_{i}+g S_{i}\right)=f\left(1 S_{i}+g S_{i}\right)$. Do fato de $1 S_{i}+g S_{i} \in \Delta$ para todo $i \in I$, pois $\varepsilon\left(1 S_{i}+g S_{i}\right)=\varepsilon\left(1 S_{i}\right)+\varepsilon\left(g S_{i}\right)=\overline{1}+\overline{1}=\overline{0}$, e $\varphi(f)=\left.f\right|_{\Delta}: \Delta \rightarrow \mathcal{F}_{T}(G)$, segue que $B_{i}+g B_{i} \in \mathcal{F}_{T}(G)$, para todo $i \in I$, ou seja, $B \in\left(\mathcal{A}_{T}(G)\right)^{I}$.

2. $B_{i}+B_{j}=f\left(S_{i}\right)+f\left(S_{j}\right)=f\left(S_{i}+S_{j}\right)=f\left(1 S_{i}+1 S_{j}\right)$, com $1 S_{i}+1 S_{j} \in \Delta$ para todo $i, j \in I$, e deste modo $B_{i}+B_{j} \in \mathcal{F}_{T}(G)$, para todo $i, j \in I$.

3. $\mathbf{B} \in \prod_{i \in I}(\mathcal{P}(G))^{S_{i}} \Rightarrow B_{i} \in(\mathcal{P}(G))^{S_{i}}, \forall i \in I \Rightarrow S_{i} B_{i}=B_{i}, \forall i \in I$.

De 1., 2. e 3. concluímos que $\mathcal{K} \subset \mathcal{H}$.

- Por outro lado, tomando $\mathbf{B} \in \mathcal{H}$, temos que $\mathbf{B}=\left(B_{i}\right)_{i \in I}$ é tal que $S_{i} B_{i}=$ $B_{i}$ para todo $i \in I, \operatorname{logo} \boldsymbol{B} \in \prod_{i \in I}(\mathcal{P}(G))^{S_{i}}$. Como $\phi$ é um isomorfismo existe $f \in \operatorname{Hom}_{\mathbb{Z}_{2} G}\left(\mathbb{Z}_{2}(G / \mathcal{S}), \mathcal{P}(G)\right)$ tal que $\phi(f)=\left(B_{i}\right)_{i \in I}$. Logo, $B_{i}=f\left(S_{i}\right)$, para todo $i \in I$. Além disso, para qualquer $x \in \Delta, x$ é a soma de um número par de elementos da forma $\sum_{j \in J \subset I} \overline{1} g_{j} S_{j}$. Suponha (sem perda de generalidade) que $J=\{1, \ldots, k\}$ e $x=\overline{1} g_{1} S_{1}+\ldots+\overline{1} g_{k} S_{k}$, com $k$ um número par, desta forma segue 
que $\varphi(f)(x)=\left.f\right|_{\Delta}(x)=f(x)=f\left(\sum_{j \in J} \overline{1} g_{j} S_{j}\right)=\sum_{j \in J} f\left(\overline{1} g_{j} S_{j}\right)=\sum_{j \in J} \overline{1} g_{j} f\left(S_{j}\right)=$ $\sum_{j \in J} g_{j} f\left(S_{j}\right)=\sum_{j \in J} g_{j} B_{j}=\sum_{j \in J}\left(g_{j} B_{j}+B_{j}+B_{j}\right)=\left[\sum_{j \in J}\left(g_{j} B_{j}+B_{j}\right)+\sum_{j \in J} B_{j}\right] \in \mathcal{F}_{T}(G)$, pois $\left(g_{j} B_{j}+B_{j}\right) \in \mathcal{F}_{T}(G)$, para todo $j \in J ;\left(B_{j}+B_{i}\right) \in \mathcal{F}_{T}(G)$, para todo $i, j \in J$, e $J$ têm um número par de elementos. Logo $\mathcal{H} \subset \mathcal{K}$, e portanto temos que $\mathcal{K}=\mathcal{H}$. Assim, temos bem definida uma aplicação sobrejetora (visto que $\varphi$ é sobrejetora) $\delta_{1}:=\varphi \circ \phi^{-1}: \mathcal{H}=\mathcal{K}=\phi\left(\varphi^{-1}\left(\operatorname{Hom}_{\mathbb{Z}_{2} G}\left(\Delta, \mathcal{F}_{T}(G)\right)\right) \rightarrow \operatorname{Hom}_{\mathbb{Z}_{2} G}\left(\Delta, \mathcal{F}_{T}(G)\right)\right.$ Mostremos que $\operatorname{Ker}\left(\varphi \circ \phi^{-1}\right) \simeq \mathbb{Z}_{2}$. Sabemos que $\operatorname{Ker}(\varphi) \simeq \mathbb{Z}_{2}$. Suponhamos

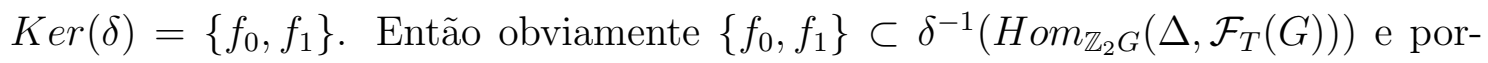
tanto $\left\{\phi\left(f_{0}\right), \phi\left(f_{1}\right)\right\} \subset \phi\left(\varphi^{-1}\left(H_{o m} \mathbb{Z}_{2} G\left(\Delta, \mathcal{F}_{T}(G)\right)\right)\right)=\mathcal{H}$ e além disso $\left\{\phi\left(f_{0}\right), \phi\left(f_{1}\right)\right\} \subset$ $\operatorname{Ker}\left(\delta_{1}\right)$. Suponhamos que existe $\mathbf{B} \in \mathcal{H}$ tal que $\mathbf{B} \in \operatorname{Ker}\left(\delta_{1}\right)$ mas $\mathbf{B} \notin\left\{\phi\left(f_{0}\right), \phi\left(f_{1}\right)\right\}$, então $\mathbf{B}=\phi\left(f_{3}\right)$, com $f_{3} \notin\left\{f_{0}, f_{1}\right\}$ e $0=\delta_{1}(\mathbf{B})=\varphi\left(\phi^{-1}(\mathbf{B})\right)=\varphi\left(\phi^{-1}\left(\phi\left(f_{3}\right)\right)\right)=$ $\varphi\left(f_{3}\right)$, ou seja, $f_{3} \in \operatorname{Ker}(\varphi)=\left\{f_{0}, f_{1}\right\}$, o que é uma contradição, e portanto temos que $\operatorname{Ker}\left(\delta_{1}\right)=\left\{\phi\left(f_{0}\right), \phi\left(f_{1}\right)\right\} \simeq \mathbb{Z}_{2}$.

Obtemos então a sequência exata desejada (onde por abuso estamos denotando $\varphi \circ \phi^{-1}$ simplesmente por $\left.\delta\right)$

$$
0 \longrightarrow \mathbb{Z}_{2} \longrightarrow \mathcal{H} \stackrel{\delta}{\longrightarrow} H^{1}\left(G, \mathcal{S} ; \mathcal{F}_{T}(G)\right) \longrightarrow 0
$$

(2) Provemos agora a segunda afirmação, isto é, que se $\mathbf{B}=\left(B_{i}\right)_{i \in I}$ é tal que $\delta(\mathbf{B})=$ $\zeta \in H^{1}\left(G, \mathcal{S} ; \mathcal{F}_{T}(G)\right)$ então $\xi=\operatorname{res}_{G}^{(G, \mathcal{S})}(\zeta) \in H^{1}\left(G ; \mathcal{F}_{T}(G)\right)$ é representado por $B_{1}$, mais precisamente $\delta\left(\left[B_{1}\right]\right)=\xi=\operatorname{res}_{G}^{(G, \mathcal{S})}(\zeta)$.

Lembremos que $\delta(\mathbf{B})=\zeta$, com $\mathbf{B} \in \mathcal{H}$ implica que $\mathbf{B}=\phi(h)$, com $h \in \varphi^{-1}\left(\operatorname{Hom}_{\mathbb{Z}_{2} G}\left(\Delta, \mathcal{F}_{T}(G)\right)\right)$, ou seja, $\left.h\right|_{\Delta}=\varphi(h) \in \operatorname{Hom}_{\mathbb{Z}_{2} G}\left(\Delta, \mathcal{F}_{T}(G)\right)$, e te$\operatorname{mos} \mathbf{B}=\phi(h)=\left(h\left(S_{i}\right)\right)_{i \in I}$.

Ainda, $\zeta=\delta(\mathbf{B})=\left(\varphi \circ \phi^{-1}\right)(\mathbf{B})=\varphi \circ \phi^{-1}(\phi(h))=\left.h\right|_{\Delta}$. Daí pelo Corolário 6.2.1, $\xi=\operatorname{res}_{G}^{(G, \mathcal{S})}(\zeta)=\operatorname{res}_{G}^{(G, \mathcal{S})}\left(\left.h\right|_{\Delta}\right)=\left(\rho \circ \tau^{*}\right)\left(\left.h\right|_{\Delta}\right)=\rho\left(\left.\left.h\right|_{\Delta} \circ \tau\right|_{\mathcal{G}}\right)=\rho\left(\left.(h \circ \tau)\right|_{\mathcal{G}}\right)$. Mas dado $1 . g \in \mathbb{Z}_{2} G,(h \circ \tau)(1 . g)=h\left(g S_{1}\right)=g h\left(S_{1}\right)=g B_{1}$ e $\left[g B_{1}\right]=\left[B_{1}\right]$, pois $g B_{1}+B_{1} \in \mathcal{F}_{T}(G)$, visto que $B_{1} \in \mathcal{A}_{T}(G)$.

Proposição 6.2.3. Sejam $(G, \mathcal{S})$ um par grupo, $\mathcal{S}=\left(S_{i}\right)_{i \in I}$, I finito, $S$ e $T$ subgrupos de $G$, e suponhamos $H^{1}\left(G, \mathcal{S} ; \mathcal{F}_{T}(G)\right) \simeq \mathbb{Z}_{2}$. Se $\mathcal{S}^{*}=\left(S_{i}^{*}\right)_{i \in I}$ é uma outra familia de subgrupos de $G$, onde $S_{i}^{*}=S^{g_{i}}=g_{i} S_{i} g_{i}^{-1}, T^{*}$ é comensurável com $T$ e $S^{*} \sim S$, então $\operatorname{sing}_{\left(G, \mathcal{S}^{*}\right)}\left(T^{*}, S^{*}\right)=0$ se, e somente se $\operatorname{sing}_{(G, \mathcal{S})}(T, S)=0$.

Demonstração: Consideraremos apenas o caso em que $\mathcal{S} \neq \emptyset$, o outro caso é similar.

Vimos que $\mathcal{F}_{T}(G)$ depende apenas da classe de comensurabilidade de $T$, de modo que $\mathcal{F}_{T}(G)=\mathcal{F}_{T^{*}}(G)$ e $\operatorname{sing}_{(G, \mathcal{S})}(T, S)=\operatorname{sing}_{(G, \mathcal{S})}\left(T^{*}, \mathcal{S}\right)$ (vide Lema 6.2.2).

(i) Mostremos que $H^{1}\left(G, \mathcal{S}^{*} ; \mathcal{F}_{T}(G)\right) \simeq H^{1}\left(G, \mathcal{S} ; \mathcal{F}_{T}(G)\right) \simeq \mathbb{Z}_{2}$. 
Seja $\sigma: \mathbb{Z}_{2}\left(G / \mathcal{S}^{*}\right) \rightarrow \mathbb{Z}_{2}(G / \mathcal{S})$ definida nos geradores por $\sigma\left(1 . S_{i}^{*}\right)=g_{i} S_{i}$ e estendida por linearidade. Claramente $\sigma$ é um $\mathbb{Z}_{2} G$-homomorfismo. Além disso, $\sigma$ é um isomorfismo pois

$$
\begin{aligned}
\sigma^{-1}: \mathbb{Z}_{2}(G / \mathcal{S}) & \rightarrow \mathbb{Z}_{2}\left(G / \mathcal{S}^{*}\right) \\
1 . S_{i} & \mapsto g_{i}^{-1} S_{i}^{*}
\end{aligned}
$$

é o inverso de $\sigma$.

Temos também que $\sigma$ preserva aumentação, ou seja, $\varepsilon \circ \sigma=\varepsilon_{*}$

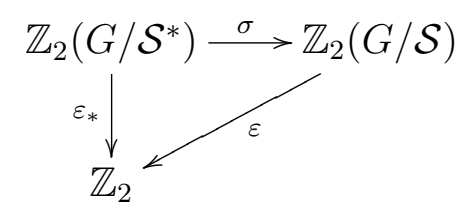

onde $\varepsilon$ e $\varepsilon_{*}$ são os homomorfismos aumentação. Basta ver isso nos geradores :

$(\varepsilon \circ \sigma)\left(1 . S_{i}^{*}\right)=1=\varepsilon_{*}\left(1 . S_{i}^{*}\right)$. Daí, $u \in \operatorname{Ker}\left(\varepsilon_{*}\right) \stackrel{\text { not. }}{=} \Delta_{*} \Leftrightarrow \sigma(u) \in \operatorname{Ker}(\varepsilon)=\Delta$, e temos bem definido o $\mathbb{Z}_{2} G$-homomorfismo

$$
\left.\sigma\right|_{\Delta_{*}}: \operatorname{Ker}\left(\varepsilon_{*}\right)=\Delta_{*} \mapsto \operatorname{Ker}(\varepsilon)=\Delta .
$$

Sabemos que, a menos de isomorfismo, tem-se $H^{1}\left(G, \mathcal{S} ; \mathcal{F}_{T}(G)\right)=\operatorname{Hom}_{\mathbb{Z}_{2} G}\left(\Delta, \mathcal{F}_{T}(G)\right)$ e $H^{1}\left(G, \mathcal{S}^{*} ; \mathcal{F}_{T}(G)\right)=\operatorname{Hom}_{\mathbb{Z}_{2} G}\left(\Delta_{*}, \mathcal{F}_{T}(G)\right)$.

Agora

$$
\begin{aligned}
\bar{\sigma}: \operatorname{Hom}_{\mathbb{Z}_{2} G}\left(\Delta, \mathcal{F}_{T}(G)\right) & \rightarrow \operatorname{Hom}_{\mathbb{Z}_{2} G}\left(\Delta_{*}, \mathcal{F}_{T}(G)\right) \\
h & \mapsto \bar{\sigma}(h):=\left.h \circ \sigma\right|_{\Delta_{*}}
\end{aligned}
$$

nos dá o isomorfismo desejado.

Em particular, se $\zeta \in H_{\operatorname{lom}_{\mathbb{Z}_{2} G}}\left(\Delta, \mathcal{F}_{T}(G)\right) \equiv H^{1}\left(G, \mathcal{S} ; \mathcal{F}_{T}(G)\right) \simeq \mathbb{Z}_{2}$ é o gerador então $\zeta_{*}:=\bar{\sigma}(\zeta)=\left.\zeta \circ \sigma\right|_{\Delta_{*}}$ é o gerador de $H^{1}\left(G, \mathcal{S}^{*} ; \mathcal{F}_{T}(G)\right)$.

(ii) Seja $\mathbf{B}=\left(B_{i}\right)_{i \in I}$ um representante de $\zeta$ em $H^{1}\left(G, \mathcal{S} ; \mathcal{F}_{T}(G)\right)$, isto é, $\mathbf{B} \in \mathcal{H}=$ $\left\{\left(B_{i}\right)_{i \in I} ; B_{i}+B_{j} \in \mathcal{F}_{T}(G)\right.$ e $\left.S_{i} B_{i}=B_{i}, \forall i, j \in I\right\}$ e $\delta(\mathbf{B})=\zeta$. Afirmamos que $B^{*}=\left(g_{i} B_{i}\right)_{i \in I}$ representa o elemento não nulo $\zeta_{*}$ de $H^{1}\left(G, \mathcal{S}^{*} ; \mathcal{F}_{T}(G)\right)$. Para isso lembremos que, conforme visto na demonstração da Proposição 6.2.2, parte (1), $\zeta=\delta(\mathbf{B}) \stackrel{\text { def. } \delta}{=}\left(\varphi \circ \phi^{-1}\right)(\mathbf{B})=\varphi(f)=\left.f\right|_{\Delta}$, onde $f\left(S_{i}\right)=B_{i}$.

Também devemos ter, pela Proposição $6.2 .2, \zeta_{*}=\delta\left(\mathbf{B}^{*}\right)=\left.f_{*}\right|_{\Delta_{*}}$ para algum $f_{*} \in H_{o m} \mathbb{Z}_{2} G\left(\mathbb{Z}_{2}\left(G / \mathcal{S}^{*}\right), \mathcal{P}(G)\right), \operatorname{com} f_{*}\left(1 . S_{i}^{*}\right)=B_{i}^{*}$ e $\mathcal{H}^{*}=\left\{\left(B_{i}^{*}\right)_{i \in I} ; B_{i}^{*}+B_{j}^{*} \in\right.$ $\mathcal{F}_{T}(G)$ e $\left.S_{i}^{*} B_{i}^{*}=B_{i}^{*}\right\}$. Considere $f_{*}:=f \circ \sigma: \mathbb{Z}_{2}\left(G / \mathcal{S}^{*}\right) \rightarrow \mathcal{P}(G)$. Então $f_{*} \in \operatorname{Hom}_{\mathbb{Z}_{2} G}\left(\mathbb{Z}_{2}\left(G / \mathcal{S}^{*}\right), \mathcal{P}(G)\right)$ e $\left.f_{*}\right|_{\Delta_{*}}=\left.(f \circ \sigma)\right|_{\Delta_{*}}=\left.\left.f\right|_{\Delta} \circ \sigma\right|_{\Delta_{*}}=\left.\zeta \circ \sigma\right|_{\Delta_{*}}=\zeta_{*}$. 
Assim devemos ter $B_{i}^{*}=f_{*}\left(S_{i}^{*}\right)=f_{*}\left(1 . S_{i}^{*}\right)=(f \circ \sigma)\left(1 . S_{i}^{*}\right)=f\left(g_{i} S_{i}\right)=g_{i} f\left(S_{i}\right)=$ $g_{i} B_{i}$, isto é, $\mathbf{B}^{*}=\left(g_{i} B_{i}\right)_{i \in I}$.

(I) Vamos provar agora a proposição para $S^{*} \sim S$, em dois casos particulares :

$\mathbf{1}^{\circ}$ caso: $S^{*}=g S g^{-1}$, para algum $g \in G$.

$2^{\circ}$ caso: $S^{*}$ e $S$ são tais que $\left[S^{*}: S\right]<\infty$,

ou seja, vamos provar que se $S^{*}=g S g^{-1}$ ou $\left[S^{*}: S\right]<\infty$, então:

$\operatorname{sing}_{(G, \mathcal{S})}(T, S)=0 \Leftrightarrow \operatorname{sing}_{\left(G, \mathcal{S}^{*}\right)}\left(T, \mathcal{S}^{*}\right)=\operatorname{sing}_{\left(G, \mathcal{S}^{*}\right)}\left(T^{*}, \mathcal{S}^{*}\right)=0$.

- Suponhamos inicialmente que $\operatorname{sing}_{(G, \mathcal{S})}(T, S)=\operatorname{res}_{S}^{G}\left(\operatorname{res}_{G}^{(G, \mathcal{S})}(\zeta)\right)=0$.

Como $\mathbf{B}=\left(B_{i}\right)_{i \in I} \in \mathcal{H}$ representa o elemento $\zeta$ de $H^{1}\left(G, \mathcal{S} ; \mathcal{F}_{T}(G)\right)$, sabemos da Proposição 6.2.2, parte (2), que $\xi=\operatorname{res}_{G}^{(G, \mathcal{S})}(\zeta)$ é representado por $B_{1}$. Mais precisamente, $\delta\left(\left[B_{1}\right]\right)=\xi=\operatorname{res}_{G}^{(G, \mathcal{S})}(\zeta)$.

Da suposição de que $\operatorname{res}_{S}^{G}\left(\operatorname{res}_{G}^{(G, \mathcal{S})}(\zeta)\right)=\operatorname{sing}_{(G, \mathcal{S})}(T, S)=0$, segue que $\operatorname{res}_{S}^{G}\left(\delta\left(\left[B_{1}\right]\right)\right)=0$. Deste modo, pelo Lema 6.2 .3 existe $B_{0} \in A_{T}(G)$ tal que $B_{1}+B_{0} \in \mathcal{F}_{T}(G)$ e $B_{0}=S B_{0}$.

Queremos concluir que $\operatorname{sing}_{\left(G, \mathcal{S}^{*}\right)}\left(T, \mathcal{S}^{*}\right)=0$, ou seja, $\operatorname{res}_{\mathcal{S}^{*}}^{G}\left(\operatorname{res}_{G}^{\left(G, \mathcal{S}^{*}\right)}(\zeta)\right)=0$. Por $(i i), \zeta_{*}$ corresponde a $\mathbf{B}^{*}=\left(g_{i} B_{i}\right)_{i \in I}$ e consequentemente, pela Proposição $6.2 .2, \xi=\operatorname{res}_{G}^{\left(G, \mathcal{S}^{*}\right)}(\zeta)$ corresponde a $g_{1} B_{1}=B_{1}^{*}$. Novamente pelo Lema 6.2.3 é suficiente provar que, nos dois casos já citados, existe $B_{0}{ }^{*} \in A_{T}(G)$ tal que $B_{0}^{*}+B_{1}^{*} \in \mathcal{F}_{T}(G)$ e $S^{*} B_{0}^{*}=B_{0}^{*}$. Provemos que isso realmente ocorre:

$1^{\circ}$ caso: $\left(S^{*}=g S g^{-1}\right)$ Nesse caso tomando $B_{0}^{*}=g B_{0}$ segue que

1. $B_{0}^{*}+B_{1}^{*} \in \mathcal{F}_{T^{*}}(G)$, pois $B_{0}^{*}+B_{1}^{*}=g B_{0}+g_{1} B_{1}=g B_{0}+g_{1} B_{1}+g_{1} B_{0}+$ $g_{1} B_{0}=g B_{0}+g_{1} B_{0}+g_{1}\left(B_{1}+B_{0}\right)=g B_{0}+B_{0}+B_{0}+g_{1} B_{0}+g_{1}\left(B_{1}+\right.$ $\left.B_{0}\right) \in \mathcal{F}_{T}(G)$, uma vez que $g B_{0}+B_{0} \in \mathcal{F}_{T}(G), B_{0}+g_{1} B_{0} \in \mathcal{F}_{T}(G)$ e $g_{1}\left(B_{1}+B_{0}\right) \in \mathcal{F}_{T}(G)$.

2. $S^{*} B_{0}^{*}=g S g^{-1} g B_{0}=g S B_{0}=g B_{0}=B_{0}^{*}$.

$2^{\circ}$ caso: $\left(\left[S^{*}: S\right]<\infty\right)$

$\left[S^{*}: S\right]=k<\infty \Rightarrow S^{*}=h_{1} S \cup \ldots \cup h_{k} S,\left(h_{i}, i=1, \ldots, k\right.$, representantes das classes laterais).

Seja $S_{0}=\left\{h_{1}, \ldots, h_{k}\right\} \subset S^{*}$ e tome $B_{0}^{*}=S_{0} B_{0}$. Deste modo temos

1. $B_{1}^{*}+B_{0}^{*}=g_{1} B_{1}+S_{0} B_{0}=g_{1} B_{1}+\left(h_{1} B_{0} \cup \ldots \cup h_{k} B_{0}\right)=\left(g_{1} B_{1}+h_{1} B_{0}\right)$ $\cup \ldots \cup\left(g_{1} B_{1}+h_{k} B_{0}\right) \subseteq F_{1} T \cup \ldots \cup F_{k} T=\left(F_{1} \cup \ldots \cup F_{k}\right) T$, com $F_{1}, \ldots, F_{k}$ finitos, ou seja, $B_{1}^{*}+B_{0}^{*} \in \mathcal{F}_{T}(G)=\mathcal{F}_{T^{*}}(G)$.

2. A inclusão $B_{0}^{*} \subset S^{*} B_{0}^{*}$ é clara.

Note que $S_{0} \subset S^{*} \Rightarrow S^{*} S_{0} \subset S^{*} ; S^{*}=h_{1} S \cup \ldots \cup h_{k} S=S_{0} S$, e $S B_{0}=$ $B_{0}$. Deste modo teremos $S^{*} B_{0}^{*}=S^{*}\left(S_{0} B_{0}\right)=\left(S^{*} S_{0}\right) B_{0} \subset S^{*} B_{0}=$ $S_{0} S B_{0}=S_{0} B_{0}=B_{0}^{*}$. E com isso temos a igualdade $B_{0}^{*}=S^{*} B_{0}^{*}$. 
Logo, pelo Lema 6.2.3, tem-se $\operatorname{sing}_{\left(G, \mathcal{S}^{*}\right)}\left(T^{*}, S^{*}\right)=0$, nos dois casos afirmados. Em particular, provamos que

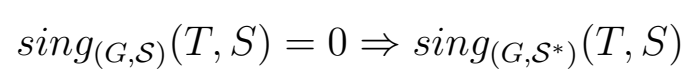

(pois basta tomar $S^{*}=S$ no $2^{\circ}$ caso, visto que obviamente $\left[S^{*}: S\right]=1$ )

- Para a recíproca, suponhamos $\operatorname{sing}_{\left(G, \mathcal{S}^{*}\right)}\left(T^{*}, S^{*}\right)=0$.

Observemos que $\mathcal{S}=\left(\mathcal{S}^{*}\right)^{*}$, usando agora $g_{i}^{-1}$, visto que $g_{i}^{-1} S_{i}^{*} g_{i}=S_{i}$. Assim, a recíproca para o caso $S^{*}=S^{g} \Leftrightarrow S=\left(S^{*}\right)^{g^{-1}}$, segue da implicação anterior, usando o par $\left(G, \mathcal{S}^{\prime}\right)=\left(G, \mathcal{S}^{*}\right)$, e $S^{\prime}=S^{*}$, pois $\operatorname{sing}_{\left(G, \mathcal{S}^{*}\right)}\left(T, S^{*}\right)=$ $0 \Rightarrow \operatorname{sing}_{\left(G,\left(\mathcal{S}^{*}\right)^{*}\right)}\left(T,\left(S^{*}\right)^{*}\right)=0$, isto é, $\operatorname{sing}_{(G, \mathcal{S})}(T, S)=0$. Vejamos a recíproca para o $2^{\circ}$ caso, isto é, $\left[S^{*}: S\right]<\infty$. Usando (6.4) e o Lema 6.2.2, temos:

$\operatorname{sing}_{\left(G, \mathcal{S}^{*}\right)}\left(T, S^{*}\right)=0 \stackrel{6.4}{\Rightarrow} \operatorname{sing}_{\left(G,\left(\mathcal{S}^{*}\right)^{*}\right)}\left(T, S^{*}\right)=0 \Rightarrow \operatorname{sing}_{(G, \mathcal{S})}\left(T, S^{*}\right)=0 \stackrel{\text { Lema } 6.2 .2}{\Rightarrow}$ $\operatorname{sing}_{(G, \mathcal{S})}(T, S)=0$.

(II) Provemos então a proposição para o caso geral.

Sejam $S^{*}$ e $S$ subgrupos quaisquer de $G$ com $S^{*} \sim S$. Então existe $g \in G$ tal que $S^{*}$ e $S^{g}$ são comensuráveis. Assim $\left[S^{*}: S^{g} \cap S^{*}\right]<\infty$ e $\left[S^{g}: S^{g} \cap S^{*}\right]<\infty$. Daí, usando (I), e o fato que $\mathcal{S}=\left(\mathcal{S}^{*}\right)^{*}$, conforme o observado, obtemos $\operatorname{sing}_{(G, \mathcal{S})}(T, S)=0 \stackrel{1^{\circ} \text { caso }}{\Leftrightarrow}$ $\operatorname{sing}_{\left(G, \mathcal{S}^{*}\right)}\left(T, S^{g}\right)=0 \stackrel{2^{\circ} \text { caso }}{\Leftrightarrow} \operatorname{sing}_{(G, \mathcal{S})}\left(T, S^{g} \cap S^{*}\right)=0 \stackrel{2^{\circ} \stackrel{\text { caso }}{\Leftrightarrow} \operatorname{sing}\left(G, \mathcal{S}^{*}\right)}{ }\left(T, S^{*}\right)=0$.

\subsection{O Invariante $E\left(G, \mathcal{S}, \mathcal{F}_{T}(G)\right)$}

O invariante cohomológico $E(G, \mathcal{S}, M), M$ um $\mathbb{Z}_{2} G$-módulo qualquer, foi definido em [2]. Se $T$ é um subgrupo de $G$ podemos considerar $M=\mathcal{F}_{T}(G)$ e o invariante $E\left(G, \mathcal{S}, \mathcal{F}_{T}(G)\right)$. Esse invariante é uma extensão de $\tilde{E}(G, S)$, uma vez que:

$$
\tilde{E}(G, S)=E\left(G,\{S\}, \mathcal{F}_{S}(G)\right) .
$$

Como vimos, alguns resultados sobre decomposição de grupos foram obtidos envolvendo $\tilde{E}(G, S)$. Assim pode ser interessante estudar $E\left(G, \mathcal{S}, \mathcal{F}_{T}(G)\right)$ para ver se esse invariante nos fornece algum resultado sobre decomposição de grupos envolvendo a família $\mathcal{S}$ (por exemplo, sobre decomposição adaptada).

Nesse sentido, nós finalizamos esse trabalho apresentando uma cota superior para $E\left(G, \mathcal{S}, \mathcal{F}_{T}(G)\right)$ (quando $(G, \mathcal{S})$ é um $P D^{n}$-par e $T$ é um $P D^{n-1}$-subgrupo de $G$ ).

Definição 6.3.1. Sejam $(G, \mathcal{S})$ um par grupo, onde $\mathcal{S}=\left(S_{i}\right)_{i \in I}$ é uma família não vazia de subgrupos de $G$, não necessariamente distintos com $\left[G: S_{i}\right]=\infty$, para todo $i \in I$, e Mum $\mathbb{Z}_{2} G$-módulo. Define-se

$$
E(G, \mathcal{S}, M)=1+\operatorname{dim}_{\mathbb{Z}_{2}} \operatorname{ker}\left(\operatorname{res}_{\mathcal{S}}^{G}\right),
$$


sendo res $\operatorname{re}_{\mathcal{S}}^{G}: H^{1}(G ; M) \rightarrow \prod_{i \in I} H^{1}\left(S_{i} ; M\right) ;[f] \mapsto \operatorname{res}_{\mathcal{S}}^{G}([f])=\left(\operatorname{res}_{i}[f]\right)_{i \in I}, \quad r e s_{i}:$ $H^{1}(G ; M) \rightarrow H^{1}\left(S_{i} ; M\right)$ a aplicação restrição induzida (no nível 1 de cohomologia) da inclusão $S_{i} \hookrightarrow G$.

Proposição 6.3.1. Dado um par grupo $(G, \mathcal{S})$, se $(G, \mathcal{S})$ é um $P D^{n}$-par com $\mathcal{S} \neq \emptyset$ $\left(\left[G: S_{i}\right]=\infty\right.$, para todo $\left.i \in I\right)$ e T é um $P D^{n-1}$-subgrupo de $G$ então $E\left(G, \mathcal{S}, \mathcal{F}_{T}(G)\right) \leq 2$.

Demonstração: Considere a sequência exata longa para o par $(G, \mathcal{S})$ com coeficientes em $\mathcal{F}_{T}(G)$

$$
\begin{aligned}
& \quad 0 \rightarrow H^{0}\left(G ; \mathcal{F}_{T}(G)\right) \rightarrow \prod_{i \in I} H^{0}\left(S_{i} ; \mathcal{F}_{T}(G)\right) \stackrel{\delta}{\rightarrow} H^{1}\left(G, \mathcal{S} ; \mathcal{F}_{T}(G)\right) \stackrel{J}{\rightarrow} H^{1}\left(G ; \mathcal{F}_{T}(G)\right) \stackrel{\text { res }}{\rightarrow} \\
& \prod_{i \in I} H^{1}\left(S_{i} ; \mathcal{F}_{T}(G)\right) \rightarrow \cdots
\end{aligned}
$$

Da Proposição 4.2.1 temos que $H^{1}\left(G, \mathcal{S} ; \mathcal{F}_{T}(G)\right) \simeq \mathbb{Z}_{2}$. Como a sequência é exata, $\operatorname{ker}\left(r e s_{S}^{G}\right) \simeq \operatorname{Im}(J)$. Assim temos que $\operatorname{dim}\left(\operatorname{ker}\left(r e s_{S}^{G}\right)\right)=0$ ou $\operatorname{dim}\left(\operatorname{ker}\left(r e s_{S}^{G}\right)\right)=1 \mathrm{e}$ portanto $E\left(G, \mathcal{S}, \mathcal{F}_{T}(G)\right) \leq 2$.

Exemplo 6.3.1. Seja $(G, S)$ o $P D^{2}$-par dado no Exemplo 3.1.3. Se T é um $P D^{1}$ - subgrupo de $G$ então $E\left(G, \mathcal{S}, \mathcal{F}_{T}(G)\right) \leq 2$.

Mais geralmente

Exemplo 6.3.2. Se $X$ é uma superfície compacta da qual retiramos $r$ discos abertos $D_{i}$ com $\partial D_{i}=S_{i}^{1}, i=1, \ldots, r$ então o par $(G, \mathcal{S})$, onde $G=\pi_{1}(X)$ e $\mathcal{S}=\left(\pi_{1}\left(S_{i}^{1}\right)\right)_{I \in I}$, é um $P D^{2}$-par. Logo, se $T$ é um $P D^{1}$-subgrupo de $G$, temos $\left(G, \mathcal{S}, \mathcal{F}_{T}(G)\right) \leq 2$. 


\section{REFERÊNCIAS BIBLIOGRÁFICAS}

[1] ANDRADE, M.G.C.; FANTI, E.L.C. A note about splittings of groups and commensurability under a cohomological point of view. preprint.

[2] ANDRADE, M.G.C.; FANTI E.L.C. A relative cohomological invariant for group pairs. Manuscripta Mathematica. 83, 1-18, 1994.

[3] ANDRADE, M.G.C.; DACCACH, J. A.; FANTI, E.L.C. On certain relative cohomological invariants. International Journal of Pure and Applied Mathematics. 21, 335-352, 2005.

[4] ANDRADE, M.G.C.; DACCACH, J. A.; FANTI, E.L.C. On relative cohomology of groups. Revista de Matemática e Estatística. 17, 275-288,1999.

[5] ANDRADE, M.G.C.; FANTI, E.L.C.; PAPANI, F.M.G. A relative invariant, duality and splittings of groups. Revista de Matemática e Estatística. 21, 131-141, 2003.

[6] ANDRADE, M.G.C; FANTI, E.L.C.; SILVA, F. S. M. S. Another characterization for a certain invariant for a group pair. International Journal of Pure and Applied Mathematics. v.35, p.349- 356, 2007.

[7] BIERI, R. Gruppen Mit Poincarè Dualität. Comm. Math. Helv. v.47, p.373-396, 1972.

[8] BIERI, R. Homological Dimension of Discrete Groups. Queen Mary College Notes. London, 1976.

[9] BIERI, R., ECKMANN, B. Groups with Homological Duality Generalizing Poincaré Duality.. Inventiones Math v.20, p.103-124, 1973.

[10] BIERI, R., ECKMANN, B. Relative Homology and Poincaré duality for group pairs. Journal of Pure and Applied Algebra v.13, p.277-319, 1978. 
[11] BROWN, K.S. Cohomology of Groups. 87. New York: Springer-Verlag, 1982.(Graduate Texts in Mathematics)

[12] CIOCA, D.M. Cohomologia e Ends de Grupos. Dissertação (Mestrado em Matemática)- Instituto de Biociencias Letras e Ciencias Exatas, Universidades Estadual Paulista, São José do Rio Preto. 1997.

[13] DICKS, W.; DUNWOODY, M.J. Groups acting on graphs. Cambridge University Press, Poincaré Duality, 1989.

[14] JOHnSON, F.E.A.; WALL, C.T.C. On Groups Satisfing Poincaré Duality. Annals of Math. v.96, p.592-598, 1972.

[15] HU, S.T. Introduction to Homological Algebra. San Francisco: Holden-Day Series in Mathematics, 1968.

[16] KROPHOLLER, P.H.; ROLLER, M.A. Splittings of Poincaré duality groups. Math. Z. 97, 421-438, 1988.

[17] KROPHOLLER, P.H.; ROLLER, M.A. Splittings of Poincaré duality groups II. J. London Math Soc. (2) 38, 420-420, 1988.

[18] KROPHOLLER, P. H.; ROLLER, M. A. Relative ends and duality groups. Journal of Pure and Applied Algebra 61, p.197 - 210, 1989.

[19] MASSEY, W.S. Algebraic Topology: An Introduction. New York: SpringerVerlag, 1967.

[20] MÜLLER, H. Decomposition theorems for group pairs. Math. Z. 176, 223 - 246, 1981.

[21] RICIERI, M. M. Decomposição de grupos e Invariantes ends. 2007. 90 f. Dissertação (Mestrado em Matemática)- Instituto de Biociencias Letras e Ciencias Exatas, Universidades Estadual Paulista, São José do Rio Preto. 2007.

[22] ROTMAN, J.J.; An Introduction to Homological Algebra, Academic Press, Inc., 1979.

[23] SANTOS, A. P. Cohomologia de Grupos e Invariantes Algébricos.2006 . Dissertação (Mestrado em Matemática)- Instituto de Biociencias Letras e Ciencias Exatas, Universidades Estadual Paulista, São José do Rio Preto. 2006.

[24] SCOTT, G. P. Ends of Pairs of Groups, J. Pure Appl. Algebra 11, 179-198, 1977.

[25] SCOTT, G. P.; WALL, C. T. C. Topological Methods in Group Theory. London Math. Soc. Lect. Notes Series 36, Homological Group Theory, 137 - 203, 1979. 
[26] STALLINGS, J. R. Groups Theory and 3- dimensional manifolds. Yale Univ. Press, $61 \mathrm{pp}, 1971$.

[27] STREBEL, R. A remark on subgroups of infinite index in Poincaré duality groups, Comment. Math. Helv 52, 317-324, 1977. 


\section{ÍNDICE REMISSIVO}

$D^{n}$-grupo, 47

$D^{n}$-par, 49

G-órbita, 13

$G$-ação, 11

G-conjunto, 12

$H N N$-extensão, 63

$H N N$-grupo, 63

$P D^{n}$-grupo, 48

$P D^{n}$-par, 49

ação diagonal, 29

ação livre, 12

ação trivial, 12

anel grupo, 11

aplicação aumentação, 11

aplicação de cocadeias, 3

aplicação induzida em cohomologia, 35

aplicação restrição, 38

aplicações homotópicas, 9

apresentação, 62

coextensão de escalares, 32

coindução, 33

complexo de cadeias, 2

complexo de cocadeias, 2

conjunto $T$-cofinito, 55

conjunto $T$-finito, 55

conjuntos $T$-quase invariante, 56

decomposição adaptada, 72 dimensão cohomológica, 47

equivalência de homotopia, 10

equivalênica fraca, 10

estabilizador, 14

extensão de escalares, 32

grupo de cohomologia, 28

grupo de coinvariantes, 29

grupo de dualidade, 47

grupo de dualidade de Poincaré, 48

grupo de homologia, 27

grupo de invariantes, 28

grupos comensuráveis, 57

grupos de (co)homologia absoluta, 27

grupos de (co)homologia relativa, 39

homomorfismo conexão, 5

homomorfismo indizido em cohomologia, 3

homomorfismos homotópicos, 9

homotopia, 9

ideal aumentação, 11

indução, 33

Lema de Shapiro, 38

módulo de cohomologia, 2

módulo de homologia, 2

módulo de homomorfismos, 21

módulo dualizante, 47 
módulo induzido, 33

número de ends de um grupo, 64

número de ends para par grupo, 66

o invariante $\tilde{e}(G, S), 70$

o invariante $\widetilde{E}(G, S), 71$

o invariante $E\left(G, \mathcal{S}, \mathcal{F}_{T}(G)\right), 83$

obstrução sing, 68

obstrução sing generalizada, 73

obstrução mista, 73

operação diferença simétrica, 13

par adaptado, 73

par de dualidade, 49

par de dualidade de Poincaré, 49

par Eilenberg-MacLane, 49

par grupo, 39

produto livre, 62

produto livre amalgamado, 62

produto tensorial, 25

resolução, 15

livre, 15

projetiva, 15

restrição de escalares, 31

sequência exata, 1

sequência semi-exata, 1 
Autorizo a reprodução xerográfica para fins de pesquisa.

São José do Rio Preto, $\_$_ $/$

Assinatura 